\title{
Gridlock and inefficient policy instruments
}

\author{
David Austen-Smith \\ Kellogg School of Management, Northwestern University \\ Wioletta Dziuda \\ Harris School of Public Policy, University of Chicago \\ BÅRD HARSTAD \\ Department of Economics, University of Oslo \\ Antoine Loeper \\ Department of Economics, Universidad Carlos III de Madrid
}

\begin{abstract}
Why do rational politicians choose inefficient policy instruments? Environmental regulation, for example, often takes the form of technology standards and quotas even when cost-effective Pigou taxes are available. To shed light on this puzzle, we present a stochastic game with multiple legislative veto players and show that inefficient policy instruments are politically easier to repeal than efficient instruments. Anticipating this, heterogeneous legislators agree more readily on an inefficient policy instrument. We describe when inefficient instruments are likely to be chosen, and predict that they are used more frequently in (moderately) polarized political environments and in volatile economic environments. We show conditions under which players strictly benefit from the availability of the inefficient instrument.
\end{abstract}

Keywords. Dynamic games, bargaining, political economy, political instruments.

JEL CLASSIFICATION. D78.

David Austen-Smith: dasm@kellogg . northwestern.edu

Wioletta Dziuda: wdziuda@chicago.edu

Bård Harstad: bard.harstad@econ.uio.no

Antoine Loeper: aloeper@eco.uc3m.es

This paper has benefited from the comments of seminar participants at Banco de Espana, Cerge-EI, Prague, Columbia University, Nottingham University, University of Oslo, Princeton University, University of Rochester, Stanford University, and Washington University. In particular, we are grateful to Vincent Anesi, Dan Bernhardt, Alessandra Casella, Ying Chen, John Duggan, Tassos Kalandrakis, Navin Kartik, Carlo Prato, Daniel Seidmann, Michael Ting, and Jan Zapal. David Austen-Smith is also grateful for support under the IDEX Chair, "Information, Deliberation and Collective Choice" at IAST, Toulouse. Harstad's part of the research received funding from the European Research Council (ERC) under the European Union's Horizon 2020 Research and Innovation Program (Grant 683031). Antoine Loeper gratefully acknowledges the support of the Fundacion Ramon Areces, of the Ministerio de Economía y Competitividad (Spain), grants RYC2015-18326, ECO 2016-75992-P, and MDM 2014-0431, and of the Comunidad de Madrid, grant MadEcoCM (S2015/HUM-3444). Part of this research was conducted while Antoine Loeper was a research fellow at Banco de Espana. All responsibility for any errors or views herein, however, lies exclusively with the authors.

(C) 2019 The Authors. Licensed under the Creative Commons Attribution-NonCommercial License 4.0. Available at http://econtheory.org. https://doi.org/10.3982/TE3329 


\section{InTRODUCTION}

Over the years, the economics profession has converged at a set of effective policy recommendations for a wide variety of policy areas. For example, there is widespread agreement that externalities can be more efficiently internalized with Pigou taxes than with command-and-control interventions, and that it is less distortionary to increase public revenue by eliminating economically unjustified tax deductions and exemptions than by raising tax rates. Likewise, economists have argued that some fiscal consolidation policies are less harmful than others. In practice, however, these recommendations are frequently ignored. Instead, policy makers often intervene with strictly less efficient policy instruments than others that are as readily available. This paper concerns why such apparently irrational (all else equal) political decisions might arise in a world of instrumentally rational agents.

To understand this puzzle, we present a dynamic political economy model in which the government reacts to external shocks by choosing a policy from a given menu of policies, one of which is unequivocally Pareto dominated by another available alternative. Indeed, the inefficient policy is not only Pareto dominated by the alternative at the time of adoption, but in all possible states of the world. Nevertheless, we show that the inefficient policy intervention can arise naturally from a simple legislative bargaining model without any extraneous frictions or informational asymmetries: it is the very inefficiency of the policy that makes it appealing to legislators. We further show that the availability of an inefficient policy instrument, either in addition to or in place of an efficient policy, may, at least in equilibrium, improve the welfare of all policy makers.

Our legislative bargaining model rests on three characteristics. First, legislative policy decisions involve two pivotal players. In particular, policy change requires the consent of two veto players who may disagree on when to enact or repeal an intervention. Second, the status quo policy in a dynamic, multiperiod setting is endogenous: the policy implemented in one period becomes the status quo in the next. Third, the environment is subject to shocks across time that affect the state-contingent policy preferences of the veto players in any period, thereby creating the need for periodic renegotiations.

In a closely related model with only one available policy intervention, Dziuda and Loeper (2016) observed that the three characteristics described above imply that the legislator who is pivotal for introducing the intervention is distinct from the legislator pivotal for repealing it. Consequently, the anticipation of her loss of political influence makes the legislator pivotal for implementing the intervention less inclined to introduce it in the first place, fearing that it will be hard to repeal should circumstances change. Loosely speaking, (Markov perfect) equilibrium behavior involves political gridlock. In this paper, however, we show that when players can choose not only whether, but also how, to intervene, the fear of future gridlock can induce an inefficient policy intervention: the veto player can agree on an inefficient policy instrument because the inefficient instrument will be easier to repeal. As a result, there may be states of nature in which the more economically efficient intervention is not politically feasible, whereas the less efficient intervention is approved by both veto players. 
In particular, we characterize conditions under which the inefficient policy instrument is implemented with positive probability in equilibrium. Intuitively, for an inefficient policy response to be chosen by rational legislators, it must be the case that the inefficiency is sufficiently high (respectively, low) for the more (respectively, less) interventionist legislator to approve repealing the policy in some states, so that the ease of repeal offsets the cost of inefficiency for using the inefficient policy in other states. For any strictly positive level of inefficiency, however, there exist nonpathological distributions of the state of nature under which legislators use the inefficient instrument in all equilibria.

The theory links both the political environment and the level of economic stability to the choice of policy instrument. To see this, fix the level of economic stability and consider the political environment. Suppose that the ideological distance between the legislators increases. This increases gridlock, creating room for the use of easily repealable instruments. The relationship between ideological polarization and the use of an inefficient instrument, however, turns out to be non-monotonic in our framework. The relative ease of repealing inefficient policies makes such policies attractive interventions for moderate levels of ideological polarization, but not when ideological polarization is small or when it is large. To see why, note that when the veto players have sufficiently similar preferences, they are likely to agree on when to repeal an efficient intervention. Conversely, when their preferences are sufficiently polarized, they are likely to disagree on when to repeal either type of policy intervention, in which case the strategic benefit of an inefficient intervention is too small to outweigh its cost.

To understand the effect of economic volatility, fix the political environment. For stable economic environments, the current state of nature changes little over time and any policy intervention can be expected to persist for quite some time. Consequently, the expected cost of intervening with an inefficient instrument is larger than the option value of being able to repeal such a policy more readily. Altenatively, if the current economic environment is volatile, the state of nature can vary considerably and the possibility of at least one veto player preferring to repeal an intervention relatively quickly can be high. The relative ease with which inefficient interventions are repealed, therefore, makes the use of such instruments attractive in this situation.

We show that all veto players can be strictly better off in equilibrium if the inefficient intervention is available as a policy option. Hence, our paper not only offers a rationale for the use of inefficient policy instruments, but also implies that they can be beneficial given the political constraints induced by a collective choice mechanism with multiple veto players. The less interventionist player benefits from the availability of the inefficient intervention because it is easier to repeal, and the more interventionist player benefits because it makes policy intervention politically feasible.

The model's logic can be applied to a variety of settings, including infant industry protection, environmental regulation, fiscal consolidation, and financial regulation.

Temporary protection of infant industries. Trade theorists (see, e.g., Bardhan 1971) have argued that in the presence of dynamic learning externalities, protecting an infant industry from foreign competition (or protecting an established industry from a temporary surge in foreign competition) can raise social welfare. The literature has further 
shown that subsidies are preferable to tariffs because they do not distort consumption and, because of the double-dividend effect, that tariffs are preferred to quotas and other nontariff barriers to trade. However, an important condition for these measures to be socially desirable is that they must be repealed when the industry matures (or when the temporary increase in foreign competition vanishes), although policy makers do not know ex ante when that will occur (Melitz 2005). The logic of our model suggests that the more free-trade-oriented party might prefer to protect the domestic industry with inefficient nontariff barriers to trade for fear that the more protectionist party will veto a repeal of more efficient protectionist policies. In fact, since World War II, governments have increasingly relied on nontariff barriers to trade to adapt trade policies to changes in trade flows (Bagwell and Staiger 1990).

Environmental regulation. Despite the sometimes considerable differences in perspective, economists from left to right tend to recommend Pigou taxes to regulate an externality because they are cost-effective, require little information, and offer a "double dividend" whereby emission taxes generate public revenues that allow governments to reduce other distortionary taxes. ${ }^{1}$ It is thus "a mystery," according to some economists, why the Republican party in the United States blocked such a market-based policy during the Obama administration, since doing so effectively led to the command-andcontrol regulation of power plants introduced by that administration in $2015 .^{2}$ In line with our model, however, some key Republicans may have anticipated that the administration would impose some curbs on the energy sector regardless, forcing them to use inefficient (command-and-control) instruments that would be easier to repeal once Obama's term was completed. ${ }^{3}$ Additionally, at the time of writing, the Republican administration is indeed working to repeal the regulations. It is hard to envision that the same attempt would occur if the intervention to be repealed was an efficient carbon tax combined with a lump-sum subsidy or a tax offset.

Fiscal consolidation. Consider a country deciding how to consolidate its fiscal policy after a shock has put its public debt on an unsustainable path. Although its government can do so along a variety of policy dimensions, we illustrate the logic of our argument by considering only one possibility, namely whether to focus on increasing revenues or

\footnotetext{
${ }^{1}$ Following the seminal paper by Tullock (1967), there is now a large literature on the double dividend. For a recent survey, see Jorgenson et al. (2013). In part because of the double dividend, all but 4 of 51 prominent economists surveyed in 2011 agreed that a carbon tax would be the less expensive way to reduce carbon dioxide emissions (http://www.igmchicago.org/igm-economic-experts-panel/poll-results?SurveyID= SV_9Rezb430SESUA4Y).

${ }^{2}$ See http://www.nytimes.com/2015/07/01/business/energy-environment/us-leaves-the-markets-outin-the-fight-against-carbon-emissions.html. While Pigou taxes are also relatively rare internationally, a famous exception is British Columbia, which introduced a carbon tax in 2008 . Although initially controversial, the tax has gained support from all important stakeholders thanks to the rebates in other taxes that the revenues permit (http://www.nytimes.com/2016/03/02/business/ does-a-carbon-tax-work-ask-british-columbia.html?smid=pl-share\&_r=0).

${ }^{3}$ Jim Manzi, a prominent conservative commentator on climate change, said openly that "a carbon tax would be, mostly likely, a one-way door: Once we introduce it we're stuck with it for a long time. What if our economic and climate models are too aggressive, and there is no practical economic justification for emissions reductions [...]. There are very large potential regrets to a carbon tax." (In "Conservatives, Climate Change, and the Carbon Tax", The New Atlantis, 21, 15-25, 2008).
} 
decreasing outlays. Suppose, as the existing empirical evidence suggests, that a spending cut is less contractionary and, thus, statically preferred by the policy makers to a tax increase. In that case, the less interventionist veto player is the veto player ideologically least inclined to implement a spending cut, that is, the liberal veto player. Our theory suggests that the liberal veto player may veto the spending cut and support instead a more costly tax increase in anticipation that once the fiscal situation improves, it will be easier to convince the conservative player to decrease taxes than to increase spending to its pre-crisis level. ${ }^{4}$ Consistent with this logic, there is empirical evidence indicating that fiscal adjustments based on spending cuts are longer lived than fiscal adjustments based on tax increases, i.e., the efficient adjustment is more persistent than the inefficient one (e.g., Alesina et al. 1998, Alesina and Ardagna 2013). ${ }^{5}$

Financial regulation. Financial regulation tends to respond to financial crises. For example, after the financial crisis of 2007-2008, the U.S. Congress passed the DoddFrank Wall Street Reform and Consumer Protection Act. Although hailed by many as a step in the right direction, it is a complex piece of legislation that others consider inefficient. Its provisions rely on heavy government regulation instead of price instruments recognized as more efficient at curbing systemic risk. Applying the same lens here as for the environmental regulation example, the inefficiencies can be viewed as a price paid by liberals to insure at least some regulation was implemented and, from the perspective of the more laissez faire members of Congress at the time, an acceptable intervention in response to the fallout from the crisis but one that can be more easily unpacked in economically calmer times. Consistent with this view, on June 9 2017, The Financial Choice Act, legislation that would "undo significant parts" of Dodd-Frank, passed the House 233-186.

Related literature. We are not the first researchers to offer an explanation for why governments implement inefficient policies. However, the logic that underlies our argument is, to the best of our knowledge, novel: inefficient interventions are more likely to be repealed should circumstances change, making them more likely to be accepted in the first place by all veto players. On the abstract level, there are three main features that distinguish our paper from the literature. First, the mechanism does not depend on the specificities of the economic environment, or on how the policy interacts with the private sector, or the electorate. Instead, inefficiency arises solely from the conflict of interests between legislators and the need to adapt the policy to a changing environment. Second, the inefficient policy in our model is inefficient in a static sense: there exists a policy that gives a strictly greater flow-payoff to all relevant decision makers in all states of nature. Third, the inefficient policy is not only the result of status quo inertia whereby

\footnotetext{
${ }^{4}$ See Alesina et al. (2017) for a recent literature review. The findings of that literature are still subject to intense debate, but we would like to point out that the logic of our model applies equally to the opposite case in which a tax increase is more efficient than a spending cut, the only difference being that the interventionist player is then the liberal veto player.

${ }^{5}$ An alternative explanation for why tax hikes might be chosen even when they are less efficient than spending cuts is that the latter hurt powerful constituencies such as retirees or unions. However, that explanation is harder to reconcile with empirical findings that governments whose austerity programs focus on spending cuts are no less likely to be reelected than those that focus on tax increase (Alesina et al. 1998).
} 
a previously optimal policy becomes obsolete; instead, it is actively implemented by the policy makers.

The paper closest to ours is Dziuda and Loeper (2016), who analyze a similar model except that they restrict the policy set to policies that are statically Pareto undominated for some states. They show that inefficient inertia occurs because each pivotal player fears that policy changes approved by her will be hard to repeal when she wishes to do so. As a result, the status quo can persist even when Pareto dominated. Hence, any inefficiency takes the form of status quo inertia and any policy change is a Pareto improvement. Riboni and Ruge-Murcia (2008), Zapál (2011), Duggan and Kalandrakis (2012), Bowen et al. (2017), and Dziuda and Loeper (2018) consider related models of dynamic legislative bargaining that also lead to policy inertia. Relative to these papers, our contribution is to show that adding a Pareto inefficient alternative to the policy space can mitigate policy inertia. In particular, legislators may adopt a Pareto-dominated policy change. ${ }^{6}$

Since our model requires policy makers to respond to shocks, it is related to the literature on policy reforms. Alesina and Drazen (1991) show that legislators can engage in a war of attrition over who should bear the costs of reform. Fernandez and Rodrik (1991) and Ali et al. (2017) show that uncertainty over the distributional impact of reforms may stifle them. Spolaore (2004) compares the likelihood of policy adjustment across three stylized institutions. In Strulovici (2010), an endogenous status quo bias arises if a majority learns that the new policy is beneficial for them. Anticipating this situation, policy makers may not want to try out new policies in the first place. Unlike in our model, these papers consider the policy response to a single shock after which legislators' policy preferences are fixed over time and restrict attention to efficient policy adjustments. Hence, inefficiency only takes the form of delays or failure to intervene. In contrast, legislators act without delay in our model, but the solution they adopt is inefficient.

There is also a large political science literature that explores inefficient policy making due to structural characteristics of legislative decisionmaking (e.g., the filibuster or committee structure). Important examples here include Krehbiel (1998) and Brady and Volden (2006), who explore models of gridlock, Ortner (2017), who shows that gridlock is likely near the next election, and Weingast et al. (1981) and Cox and McCubbins (2000), who analyze legislative structure and inefficient public good provision more generally.

\footnotetext{
${ }^{6}$ In a distributive environment, Bowen et al. (2014) and Anesi and Seidmann (2015) show that endogenous status quo can lead to Pareto inefficient policies, because they allow the proposer or the supporting coalition to extract greater transfers in the future. In these papers, preferences do not evolve over time, as in most of the literature on dynamic policy making with an endogenous status quo. Policy dynamics occur because the proposer changes (e.g., Baron 1996, Bernheim et al. 2006, Kalandrakis 2004, Anesi and Duggan 2018, Buisseret and Bernhardt 2017) or because the same proposer forms different coalitions over time (e.g., Diermeier and Fong 2011). In contrast, in our model, each proposer always seeks the support of the same policy maker and the qualitative nature of policy inefficiencies is, by and large, independent of the allocation of bargaining power. Acharya and Ortner (2013) obtain Pareto inefficient solution in a divide-a-dollar game because, as in our paper and for similar reasons, players cannot commit to trade-off their payoffs intertemporally. A different strand of literature assumes that the implemented policy influences future states (see Hassler et al. 2003, for example). Baldursson and von der Fehr (2007) is closer to our story, as they argue that a relatively "brown" party may prefer quotas rather than taxes, because the relatively inefficient quotas are essentially property rights that are difficult to tighten or remove later.
} 
To our knowledge, however, there is as yet no analysis that focuses directly on the deliberate strategic choice of inefficient policy change when policy change occurs.

Inefficient policy choices by a unitary actor, whether a single legislator or a fully coordinated party or group, can also be explained by aspects of the political economic environment other than legislative design. In Coate and Morris (1995), Acemoglu and Robinson (2001), and, to some extent, Glaeser and Ponzetto (2014), inefficient policy choices arise from an incumbent legislator's efforts to retain office. ${ }^{7}$ Whereas the Coate and Morris (1995) and Glaeser and Ponzetto (2014) accounts rest on asymmetric information, Acemoglu and Robinson (2001) generate policy inefficiency from a model in which distortionary transfer payments are designed to counter declining political support and influence. Tullock (1993), Grossman and Helpman (1994), Becker and Mulligan (2003), and Drazen and Limao (2008) argue that any resource transfer increases wasteful lobbying (rent-seeking) activity. By committing itself to inefficient transfers, the government can reduce the level of wasteful lobbying. More generally, there is an extensive literature on policy distortions induced through special interest groups' lobbying and campaign contribution activities (see Wright 1996 and Grossman and Helpman 2001 for overviews of the literature).

The problem at the root of our paper is related to the dynamic lack of commitment studied extensively in the literature. In Acemoglu and Robinson $(2000,2001)$ or Fearon (1998), for example, the inability of the players to commit leads to inefficient conflict (see Powell 2004 for an interesting overview). In our paper, the inability of the players to commit to repeal of certain policies leads to Pareto inefficient gridlock. Giving players access to Pareto inefficient alternatives that they frequently may want to repeal due to their inefficiency can mitigate this commitment problem.

Aidt (2003) claims that inefficient command-and-control instruments are more bureaucracy intensive and, to the extent that bureaucrats influence policy design and derive value from implementing policy, such interventions are favored by bureaucrats. Alesina and Passarelli (2014) and Masciandaro and Passarelli (2013) offer explanations of socially suboptimal policies that hinge on the median voter failing to internalize the costs and benefits to others when policies have different distributional consequences. However, both available policy choices are Pareto optimal in these papers. In contrast to these approaches, inefficiency in our model does not depend on groups, informational asymmetries, or reelection concerns.

\section{A simple example}

In this section we present a stylized example to illustrate the key mechanism and to preview some of our results. The mechanism requires two pivotal players, or legislators, $L$ and $R$, both of whom must approve any policy change. Specifically, at the start of any legislative period, nature randomly chooses one legislator to propose a change in, or maintain, the status quo policy. The other legislator has a veto right over any proposed change in the status quo. Legislators start with no intervention, denoted by $n$, and can

\footnotetext{
${ }^{7}$ See also Canes-Wrone et al. (2001) and Buisseret and Bernhardt (2018) for how electoral incentives can lead to policy distortions.
} 
intervene by introducing either an efficient instrument $p$ or an inefficient instrument $q$. The flow-payoff from policy $n$ is normalized at 0 . Intervention gives everyone a benefit $\theta$, and the costs associated with $p$ and $q$ are $w_{i}$ and $w_{i}+e_{i}$, respectively, for $i \in\{L, R\}$. We assume that $e_{i}>0$ for each $i$, so that $e_{i}$ is the additional benefit of the efficient instrument.

We can immediately make a simple observation.

Proposition 0. Suppose there is only one period. Then the inefficient policy $q$ is never implemented in equilibrium.

Suppose now that there are two periods and let $\delta>0$ be the common discount factor. The status quo in the first period is $n$ and the first-period policy becomes the status quo in the second period. Assume further that there are only two states, $\underline{\theta}$ and $\bar{\theta}>\underline{\theta}$, with $\bar{\theta}$ occurring with probability $\pi$, and suppose that the costs associated with $p$ and $q$ satisfy

$$
w_{L}<\underline{\theta}<\min \left\{w_{L}+e_{L}, w_{R}\right\} \leq \max \left\{w_{L}+e_{L}, w_{R}+e_{R}\right\}<\bar{\theta} .
$$

The last inequality in (1) means that in state $\bar{\theta}$, both players prefer any type of intervention to $n$. The first two inequalities in (1), however, imply that in state $\underline{\theta}$, the less interventionist player $R$ prefers no intervention, while the more interventionist player $L$ prefers to intervene, but only if the intervention is with the efficient instrument. In terms of our environmental application, $\bar{\theta}$ can be interpreted as the usual state of the economy in which both parties agree that environmental interventions are desirable, while $\underline{\theta}$ can be interpreted as an economic downturn that makes the $R$ party (but not the $L$ party) want to repeal any regulation that can compromise economic growth. In the case of fiscal consolidation, $\underline{\theta}$ may be interpreted as a business-as-usual state in which parties differ ideologically on whether public spending should be cut, while $\bar{\theta}$ can be interpreted as a fiscal crisis state in which both parties are willing either to cut spending or to increase taxes to bring public debt under control.

Consider the last period in this game. In state $\bar{\theta}$, both players strictly prefer $p$ to any other policy. So, independently of which player has proposal rights, $p$ is implemented in that state. What is implemented in the low state $\underline{\theta}$, however, depends on the status quo. If $n$ is the status quo, $R$ does not approve (propose or accept, depending on the allocation of proposal rights) any change in policy. Similarly, if $p$ is the status quo, $L$ does not approve of any change in policy. Finally, if $q$ is the status quo, then the two players agree that either $n$ or $p$ is better than the status quo, but they disagree on which is best. In this situation, $p$ is implemented if $L$ has the proposal power and $n$ is implemented otherwise. This reasoning implies that, in the second period, $p$ is not repealable, but $q$ can be repealable when the realized state in that period is $\underline{\theta}$.

Consider now the first period and suppose the state is $\bar{\theta}$ with status quo $n$. Both players' first-period flow-payoffs are maximized by policy $p$. But since $p$ is not repealable, $R$ may be reluctant to approve such a change in policy. In particular, $R$ does not approve $p$ in the first period if the benefit of $p$ relative to $n$ in state $\bar{\theta}$ is outweighed by the expected cost of being stuck with $p$ in state $\underline{\theta}$; that is, if

$$
\bar{\theta}-w_{R}<\delta(1-\pi)\left(w_{R}-\underline{\theta}\right) .
$$


Thus, players fail to intervene efficiently in the first period if the disagreement state $\underline{\theta}$ is relatively likely (i.e., $1-\pi$ is high), if players are patient, and if $R$ 's preference for intervention in state $\bar{\theta}$ is relatively weak compared to $R$ 's preference for no intervention in state $\underline{\theta}$. Alternatively, because $q$ is more easily repealed than $p, R$ may be willing to intervene with $q$. Denoting by $b_{L} \in[0,1]$ the probability that $L$ has the authority to make a take-it-or-leave-it policy proposal in the second period, $R$ approves an intervention $q$ in the first period if

$$
\bar{\theta}-w_{R}-e_{R} \geq \delta(1-\pi) b_{L}\left(w_{R}-\underline{\theta}\right) .
$$

In addition, since $L$ receives a higher flow-payoff from $q$ than $n$ in state $\bar{\theta}$, and the likelihood that a change from status quo $q$ to $p$ in the second period exceeds that from $n$ to $p, L$ also prefers and approves $q$ over $n$ in the first period. Finally, because players share the same ordinal policy preferences over $n$ and $q$ in both states, we have the following proposition.

Proposition 1. Suppose there are two periods and

$$
\delta(1-\pi) b_{L}\left(w_{R}-\underline{\theta}\right)+e_{R} \leq \bar{\theta}-w_{R}<\delta(1-\pi)\left(w_{R}-\underline{\theta}\right) .
$$

Then, in the first period of any subgame perfect equilibrium, the following statements hold:

(i) Intervention occurs in $\bar{\theta}$ when the policy menu is $\{n, p, q\}$, but not when the menu is $\{n, p\}$.

(ii) Both players strictly prefer menu $\{n, p, q\}$ to menu $\{n, p\}$.

The fact that efficient policies are hard to repeal can make them politically impossible to agree upon in a dynamic setting. At the same time, as part (i) of the proposition states, it is precisely because of its inefficiency that both legislators may approve a firstperiod intervention with $q$ in state $\bar{\theta}$. Additionally, as part (ii) of the proposition asserts, adding this statically Pareto-dominated choice to the policy menu may not only result in its use, but it also strictly improves equilibrium payoffs for both players.

To our knowledge, the preceding claims are new to the literature. They are, however, derived in a stylized example that raises a number of questions. For instance, the cost of instrument $q$ is limited since $q$ will always be replaced by either $n$ or $p$ in the last period. But what is the desirability of $q$ in a dynamic model when there is no last period? Furthermore, the binary state space in the example implies that instrument $p$ will never be repealed once implemented. But why should the players prefer $q$ to $p$ in a more general setting where both interventions may eventually be repealed? To explore these and several other issues further, the following section generalizes the model to an infinite number of periods and to more general distributions of states.

\section{Model}

Policies, payoffs, and players. Two infinitely lived players, $L$ and $R$, must decide in each period $t \in \mathbb{N}$ which of three policies $\{n, p, q\}$ to implement. Their preferences over these 
policies in a given period $t$ depend on the realization of the state of nature $\theta(t) \in \mathbb{R}$, and are specified as in the preceding example. That is, normalizing both players' flow-payoff from policy $n$ in any state $\theta$ to zero, $U_{i}(\theta, n)=0, i \in\{L, R\}$, player $i$ 's flow-payoffs from policies $p$ and $q$ relative to policy $n$ in $\theta$ are, respectively,

$$
\begin{aligned}
& U_{i}(\theta, p)=\theta-w_{i} \\
& U_{i}(\theta, q)=\theta-\left(w_{i}+e_{i}\right) .
\end{aligned}
$$

Thus, $e_{i}$ is the period flow-payoff gain for player $i$ from implementing $p$ instead of $q$. For simplicity, we assume that this gain is independent of $\theta$. More importantly, we assume that $e_{i}>0$ for both players. That is, intervention $p$ Pareto dominates intervention $q$ in all states of nature. Although the state of nature does not affect which intervention is best, it affects whether an intervention is needed in the first place: given the zero flow-payoff from no intervention, $n$, player $i$ gets a greater flow-payoff from policy $p$ than from policy $n$ when $\theta \geq w_{i}$, and a greater flow-payoff from $q$ than from $n$ when $\theta \geq w_{i}+e_{i}$. Importantly, unless we specify otherwise, we assume that $w_{L} \neq w_{R}$, that is, in some states of nature, players disagree whether the efficient intervention $p$ is preferred to no intervention $n$. By convention, $L$ denotes the more interventionist player, so $w_{L}<$ $w_{R} .^{8}$ At times, we refer to $w_{R}-w_{L}$ as polarization over efficient intervention.

Timing of the game. Every period $t \in \mathbb{N}$ starts with some status quo $s(t) \in\{n, p, q\}$, with $s(0)=n$. At the beginning of period $t$, both players observe the state $\theta(t) \in \mathbb{R}$. After $\theta(t)$ is observed, $L$ and $R$ must collectively choose a policy from the set $\{n, p, q\}$. We assume that one player makes a take-it-or-leave-it offer to the other regarding which policy to implement. The recognition probability for player $i$ is denoted by $b_{i}(\theta, s)$, which may depend on the current state $\theta$ and status quo $s$. We assume that $b_{i}$ is bounded below by some $\underline{b}>0$. The recognized proposer offers a policy $y(t) \in\{n, p, q\}$. If the other player, the veto player, accepts this proposal, then $y(t)$ is implemented; otherwise, the status quo $s(t)$ stays in place. The policy implemented in $t$, whether the proposal $y(t)$ or the status quo $s(t)$, generates the flow-payoff for that period, as specified in (2), and becomes the status quo in the next period, $t+1$. Each player maximizes her expected discounted payoff over the infinite horizon. The common discount factor is $\delta \in(0,1)$. For simplicity, we initially assume that $\{\theta(t): t \geq 0\}$ is distributed identically and independently over time according to some continuous cumulative distribution function (c.d.f.) $F$ with full support. This assumption is relaxed in Section 5 to permit serial correlation.

Equilibrium concept. We denote the above game by $\Gamma$ and restrict attention to Markov-perfect equilibria, referred to as "equilibria" in what follows. A Markov-perfect equilibrium is a subgame-perfect equilibrium in which players use Markov strategies. In this game, a strategy is Markov if it depends only on the current state, the current status quo, the identity of the proposer, and the current proposal at the action node of the veto player. Let $\sigma_{i}$ denote $i$ 's Markov strategy. A (pure) Markov strategy for player $i \in\{L, R\}$

\footnotetext{
${ }^{8}$ The terms "instrument" and "intervention" are used exclusively in reference to alternatives $p$ and $q$. The term "policy" may refer to any of the available alternatives, including $n$. This looseness should cause no confusion. Also, we say that a policy $p$ or $q$ is "repealed" when it is the status quo and players agree to replace it by $n$.
} 
consists of two contingent actions: a function that maps the current state and status quo into a policy proposal, conditional on $i$ being the proposer, and a function that maps the current state, status quo, and proposal into a choice over accepting or rejecting the proposal, conditional on $i$ being the veto player. ${ }^{9}$ Let $\sigma=\left(\sigma_{L}, \sigma_{R}\right)$. We briefly discuss the robustness of our restriction to Markov strategies at the end of Section 4.3. We allow for mixed strategies.

Since our interest regards the use of the inefficient instrument $q$, throughout the paper we focus on the equilibria in which $q$ is implemented. To this end, the following definition is useful.

Definition 1. Let $\sigma$ be an equilibrium of $\Gamma$. Then $\sigma$ is an instrument inefficient equilibrium (IE) if $q$ is implemented with positive probability on the equilibrium path; $\sigma$ is an instrument efficient equilibrium (EE) otherwise.

Remarks on the assumptions. Some of the assumptions are made for simplicity and relaxing them may not change the results. In particular, binary policy levels are not necessary for the results. To see this, suppose that players can choose any level of $p$ or $q$ and consider a two-period example again. The inefficient instrument $q$ will not be used in the last period and the set of states under which $p$ is repealed will be independent of its level. The inefficient $q$, however, will still be easier to repeal at any level, and the logic of our paper applies.

Three assumptions are crucial. First, the mechanics of the model rests on multiple veto players. With a unicameral legislature taking decisions under simple majority rule, the policy maker $i$ with the median $w_{i}$ would be the unique pivotal decision maker. However, multiple veto players are natural in politics. Bicameralism, supermajority requirements, presidential veto power, or powerful interests groups imply the existence of a set of veto players, or pivots, whose approval is necessary and sufficient to enact a policy change. In the case of a unicameral legislature taking decisions under a qualified majority $m \in\left(\frac{1}{2}, 1\right]$, player $L$ is such that exactly a fraction $m$ of the $w_{i}$ s are larger than $w_{L}$ and, for player $R$, exactly $m$ of the $w_{i}$ s are smaller than $w_{R}$. Thus, the degree of polarization $w_{R}-w_{L}$ increases in the majority requirement $m$.

Second, we assume away explicit side payments and state-contingent contracts. If the players could make unlimited side payments or sign state-contingent contracts, then only efficient policies would be implemented in equilibrium. ${ }^{10}$ In particular, policy $p$ would be implemented when $\theta>\left(w_{L}+w_{R}\right) / 2$, and policy $n$ would be implemented otherwise. Explicit side payments and state contingent contracts, however, are rare and often unavailable in politics. An important reason for this is that there is no independent third party that can verify a particular realization of the state and enforce any ex ante

\footnotetext{
${ }^{9}$ Note that Markov strategies are not allowed to depend on calendar time, as the date is payoff irrelevant with an infinite horizon. Mixed strategies are admissible. More formally, writing $\Delta S$ for the set of probability distributions over a set $S$, $i$ 's proposal strategy takes $\mathbb{R} \times\{n, q, p\}$ into $\Delta\{n, q, p\}$, and $i$ 's veto strategy takes $\mathbb{R} \times\{n, q, p\}^{2}$ into $\Delta\{$ accept, reject $\}$. Markov strategies are standard in the literature on dynamic bargaining with endogenous status quo.

${ }^{10}$ For results on the efficiency of state-contingent policies for the allocation of public goods, see Bowen et al. (2017).
} 
agreement. In the case of fiscal consolidation, for example, $\theta$ may capture the impact of a shock to money markets on the long term sustainability of public debt and whether fiscal consolidation can bring this debt under control. Similarly, in the environmental application, $\theta$ may capture changes in the social cost of the polluting activity and the social opportunity cost of regulating the polluting industry, and in the infant industry case, $\theta$ might be the impact of a technical innovation that affects where an infant industry stands on its learning curve. In all of these applications, it is unlikely that $\theta$ could be measured in a transparent way by a nonpartisan entity. Imperfect verifiable proxies for $\theta$ may exist (e.g., interest rate on government bonds as a proxy for the impact of a shock to money markets), but whether and under what conditions contracts contingent on such proxies improve the efficiency of the policy making is left for future research.

Third, we assume that any implemented policy stays in place until it is actively changed. This is consistent with legislative practice. Most laws and policies enacted by the U.S. Congress, for example, are permanent: they remain in effect until a new legislative action is taken. This is the case for mandatory spending policies, which include all entitlements, currently about $60 \%$ of total federal spending (Austin and Levit 2010), constitutional amendments, most statutes in the U.S. code, the Senate's rules of proceedings, and international treaties. Likewise, changes to the tax code are permanent unless legislators decide to attach a sunset provision, that is, a clause that specifies a period after which the relevant legislative act automatically expires. Historically, attaching sunset clauses to legislation has been the exception rather than the norm, and tends to occur mainly for procedural reasons. ${ }^{11}$ Nevertheless, in theory, a sunset clause can address the concern of the less interventionist player that an efficient intervention will stay in place too long. Thus, the availability of sunset clauses seems, at least prima facie, to remove the strategic value of inefficient instruments highlighted in this paper. In the working paper version, however, we present an example that shows that even when sunsets are available at no cost, the inefficient instrument might still be implemented. A careful analysis of sunsets is beyond the scope of this paper.

\section{Analysis}

The following lemma states that an equilibrium exists and that all equilibria have a relatively simple structure: in any equilibrium of $\Gamma$, players behave as if they are playing a static version of the game $\Gamma$ (a single period) with flow-payoff parameters $\left(w_{L}^{\sigma}, w_{R}^{\sigma}, e_{L}^{\sigma}, e_{R}^{\sigma}\right)$ rather than $\left(w_{L}, w_{R}, e_{L}, e_{R}\right)$.

\footnotetext{
${ }^{11}$ See, e.g., Posner and Vermeule (2002, pp. 1672, 1694, and 1701) on the permanent nature of statutes, the Senate's internal rules, or international treaties. As for tax legislation, prior to the Bush administration, the use of sunsets for changes in the tax code applied mainly to relatively small provisions known as tax extenders and were of significantly smaller scale Gale and Orszag (2003), Mooney (2004). Many sunsetted legislations are frequently extended beyond the initial duration, often because of the activities of vested interests or the legislative costs of implementing the change. Since the repeal of inefficient instruments requires legislative work only when the state of nature justifies it, and not at predetermined state-independent dates, using inefficient instruments results in lower legislative cost than sunset provisions.
} 
Lemma 1. There exist equilibria in $\Gamma$. Moreover, for any equilibrium $\sigma$, there exists a unique tuple $\left(w_{L}^{\sigma}, w_{R}^{\sigma}, e_{L}^{\sigma}, e_{R}^{\sigma}\right) \in \mathbb{R}^{4}$ such that the behavior prescribed by $\sigma$ is the same as the behavior prescribed by an equilibrium of the game in which players play a single period of $\Gamma$ with payoffs $V_{i}^{\sigma}(\theta, n)=0$ and

$$
\begin{aligned}
& V_{i}^{\sigma}(\theta, p)=\theta-w_{i}^{\sigma} \\
& V_{i}^{\sigma}(\theta, q)=\theta-\left(w_{i}^{\sigma}+e_{i}^{\sigma}\right) .
\end{aligned}
$$

All proofs are provided in the Appendix. ${ }^{12}$ We call $\left(w_{L}^{\sigma}, w_{R}^{\sigma}, e_{L}^{\sigma}, e_{R}^{\sigma}\right)$ the continuation payoff parameters induced by $\sigma$ and note that they reflect players' strategic preferences in the equilibrium, that is, their policy preferences given continuation play $\sigma$, as distinct from their exogenous "ideological" preference parameters $\left(w_{L}, w_{R}, e_{L}, e_{R}\right)$. Note that $V_{i}^{\sigma}(\theta, p)-V_{i}^{\sigma}(\theta, q)=e_{i}^{\sigma}$, so $e_{i}^{\sigma}$ represents the continuation payoff difference for player $i$ from some period $t$ onward from implementing $p$ instead of $q$ in that period, given continuation play $\sigma$. Hence, $e_{i}^{\sigma}$ reflects $i$ 's preferences over the efficient and the inefficient intervention in the dynamic game.

To understand the intuition for Lemma 1, consider the continuation payoff gain for player $i$ from implementing $p$ instead of $n$ in some $t$. Player $i$ gets a flow-payoff gain of $\theta(t)-w_{i}$ in period $t$, plus the expected continuation payoff gain from having status quo $p$ instead of $n$ in $t+1$. Since $\{\theta(t): t \in \mathbb{N}\}$ is independent and identically distributed (i.i.d.), this expected continuation payoff gain does not depend on $\theta(t)$, but only on the anticipated play $\sigma$ and, hence, is simply a constant $C^{\sigma}$. The continuation payoff gain from implementing $p$ instead of $n$ in $t$ is, therefore, $\theta(t)-w_{i}+C^{\sigma}$, so $w_{i}^{\sigma}$ is given by $w_{i}-C^{\sigma}{ }^{13}$

The main goal of this paper is to understand the strategic underpinnings of IE and why they can be beneficial. Therefore, for the sake of exposition, we focus only on IE. We first derive their properties (Section 4.1) and then the conditions under which they exist (Section 4.2).

Before we proceed, however, let us say a few words about the EE. By definition, in any EE, the inefficient instrument is not used; hence, EE are essentially equivalent to the equilibria of the two-alternative game of Dziuda and Loeper (2016). They show that in such equilibria, the more pro-intervention player distorts her votes in favor of $p$, and the less pro-intervention player distorts her votes in favor of $n$, that is, $w_{L}^{\sigma}<w_{L}<w_{R}<w_{R}^{\sigma}$. As a result, even EE are inefficient in that there is excessive status quo inertia: a policy that was once adequate for the environment becomes obsolete, but players do not agree

\footnotetext{
${ }^{12}$ Duggan and Kalandrakis (2012) provide a very general existence result for dynamic bargaining games with an endogenous status quo. But to apply their result directly here requires violating our assumption that $U(\theta, p)-U(\theta, q)$ is constant in $\theta$. Although introducing some noise to the payoffs, to ensure the difference is not locally constant, and letting that noise tend to zero is possible, the result would be a correlated equilibrium that obscures the particular trade-offs of interest here.

${ }^{13}$ If the process $\{\theta(t): t \in \mathbb{N}\}$ is serially correlated, $C^{\sigma}$ becomes a function of $\theta(t)$, whose variations depend both on the nature of the serial correlation, and on the set of types for which status quo $n$ and $p$ lead to different outcomes on the path of $\sigma$. In particular, the continuation payoff gain from implementing $p$ instead of $n$, i.e., $\theta(t)-w_{i}+C^{\sigma}(\theta(t))$, might become non-monotone in $\theta(t)$, possibly leading to equilibria that are not in threshold strategies.
} 
to change it. This kind of inefficiency, however, does not explain the puzzle outlined in the Introduction, namely, why interventions that are inefficient in any state of the world are implemented. So IE differ from EE not in whether the equilibrium path is inefficient, but in the nature of inefficiency.

\subsection{Properties of IE}

The following proposition summarizes the main qualitative properties of any IE.

Proposition 2. Let $\sigma$ be an IE. Then the corresponding continuation payoff parameters $\left(w_{L}^{\sigma}, w_{R}^{\sigma}, e_{L}^{\sigma}, e_{R}^{\sigma}\right)$ satisfy the following inequalities for some $i, j \in\{L, R\}, i \neq j$.

(i) The set of states at which $j$ prefers to intervene with $p$ is a proper subset of those at which i prefers to intervene with $p$ :

$$
w_{i}^{\sigma}<w_{j}^{\sigma} .
$$

(ii) Player i prefers to intervene with $p$ rather than $q$, and $j$ weakly prefers to intervene with $q$ rather than $p$ :

$$
e_{i}^{\sigma}>0 \text { and } e_{j}^{\sigma} \leq 0 .
$$

(iii) The set of states at which both $i$ and $j$ prefer to repeal $p$ is a proper subset of those at which they both prefer to repeal q:

$$
\min _{k \in\{L, R\}}\left(w_{k}^{\sigma}\right)<\min _{k \in\{L, R\}}\left(w_{k}^{\sigma}+e_{k}^{\sigma}\right) .
$$

Part (i) of Proposition 2 states that in any IE, one player $i \in\{L, R\}$ is strictly more interventionist than the other player $j$. Part (ii) states that, in equilibrium, players disagree on the appropriate intervention. In all states, player $i$ prefers using the efficient instrument $p$ to the inefficient instrument $q$, whereas player $j$ weakly prefers intervening with $q$ rather than $p$. Finally, part (iii) implies that, consistent with the intuition provided in the Introduction, the inefficient instrument $q$ is easier to repeal than the efficient instrument $p$. To see this, assume $p$ is the status quo. Then $e_{i}^{\sigma}>0$ implies player $i$ always vetoes proposal $q$ and, by definition of $w_{k}^{\sigma}$, at least one player vetoes the proposal $n$ for all states above $\min _{k \in\{L, R\}}\left(w_{k}^{\sigma}\right)$. Hence, in all such states, the status quo $p$ remains in place. Conversely, for all states below $\min _{k \in\{L, R\}}\left(w_{k}^{\sigma}\right)$, both players prefer $n$ to any instrument, so $p$ is repealed. Both players, however, prefer $n$ to $q$ in all states below $\min _{k \in\{L, R\}}\left(w_{k}^{\sigma}+e_{k}^{\sigma}\right)$. So (iii) implies that $q$ is repealed on a larger set of states than is $p$.

To understand the policy dynamics implied by Lemma 1 and Proposition 2, let us consider first the equilibria in which $e_{j}^{\sigma}<0$, that is, equilibria in which $j$ strictly prefers to intervene with the inefficient instrument. In that case, there are only two possible equilibrium paths, illustrated by Figures 1 and 2, depending on whether $\max _{k}\left\{w_{k}^{\sigma}+\right.$ $\left.e_{k}^{\sigma}\right\}<w_{j}^{\sigma}$ (Figure 1) or $w_{j}^{\sigma}<\max _{k}\left\{w_{k}^{\sigma}+e_{k}^{\sigma}\right\}$ (Figure 2). For concreteness, in Figures 1 and 2 we illustrate these two possibilities for the equilibria in which $i=L$ and $j=R .{ }^{14}$ States

\footnotetext{
${ }^{14}$ Since $w_{L}<w_{R}$, it is natural to conjecture that necessarily $i=L$, but equilibria with $i=R$ may arise as well. See the discussion at the end of this section.
} 


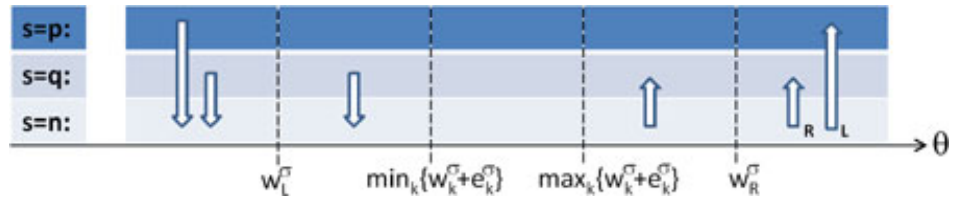

FIgURE 1. An example of IE with both players choosing $q$ for some states.

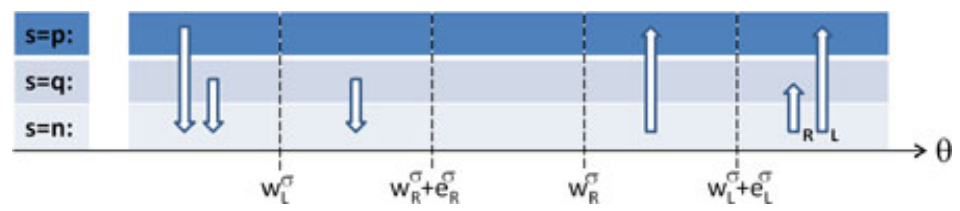

FIgURE 2. An example of IE with both players choosing $p$ for some states.

are measured along the horizontal axis and policies are indicated by the three shaded bars above this axis. An arrow identifies an equilibrium policy change, conditional on the status quo, for any realized state within the same interval of states as that in which the arrow is drawn. For intervals of states where there is no arrow drawn, there is no policy change on the equilibrium path. The status quo at the start of any period in which there is a policy change is indicated by the origin of the relevant arrow; the arrowhead indicates the policy instrument chosen to replace the status quo.

In Figure 1, for example, suppose that the status quo going into the current period is $n$ and the realized state is $\theta \in\left(\max _{k}\left\{w_{k}^{\sigma}+e_{k}^{\sigma}\right\}, w_{R}^{\sigma}\right)$. Player $L$ prefers intervening with $p$ to intervening with $q$, but state $\theta$ is high enough so that she prefers intervening with either instrument to staying with $n$. Player $R$ prefers intervening with $q$, as the expected benefit from $q$ being easier to repeal outweighs the inefficiency cost, but the difficulty of repealing $p$ makes her prefer no intervention $n$ to $p$. Hence, $q$ is the only alternative that dominates $n$ for both players in that state. As a result, $q$ is implemented independently of who is the proposer, as indicated by the upward-pointing arrow. Alternatively, if $\theta>w_{R}^{\sigma}$, the intervention is so desirable that both players prefer intervening with either policy instrument. Since they differ with respect to their ordering of $p$ and $q$, the implemented policy depends on who has proposal power. This is indicated by labeling the upward-pointing arrows for such states by the proposer's identity. When the status quo is either $p$ or $q$, the downward-pointing arrows indicate those states in which the relevant instrument is repealed. As stated in Proposition 2, $q$ is repealed for a larger set of states.

Figure 2 differs from Figure 1 only in that the less interventionist player, $R$, is willing to accept $p$ in some states for which $L$ is unwilling to accept the inefficient alternative $q$. As a result, for $\theta \in\left(w_{R}^{\sigma},\left\{w_{L}^{\sigma}+e_{L}^{\sigma}\right\}\right), R$ is forced to propose $p$ and, therefore, $p$ is implemented independently of who has proposal power.

In an equilibrium in which $e_{j}^{\sigma}=0$, when it exists, player $j$ is indifferent between intervening with $p$ and $q$, so the equilibrium may involve some mixing and the paths depicted in Figures 1 and 2 must be amended. Consider again the case in which $j=R$, as the other case is analogous. In Figures 1 and 2, whenever $n$ is replaced by $q$ 
(for $\theta>\max _{k}\left\{w_{k}^{\sigma}+e_{k}^{\sigma}\right\}$ ), it is because either $R$ is the proposer and chooses the intervention that is better for her or $L$ is the proposer and proposes $q$ because $R$ would not accept $p$. In an equilibrium with $e_{R}^{\sigma}=0$, however, $R$ is indifferent between $q$ and $p$, so in these states, $n$ may be replaced by $p$ with positive probability instead. Similarly, whenever $q$ remains in place in Figures 1 and 2 (for $\theta>\min _{k}\left\{w_{k}^{\sigma}+e_{k}^{\sigma}\right\}$ ), $R$ may agree to replace it with $p$ (given she is indifferent as $e_{R}^{\sigma}=0$ ); hence, additional arrows from $q$ to $p$ should be drawn.

Since $w_{L}<w_{R}$, it is natural to conjecture that in any equilibrium, $w_{L}^{\sigma}<w_{R}^{\sigma}$, that is, $i=L$ and $j=R$ in Proposition 2, as we assumed in Figures 1 and 2. In addition, one can indeed show that for any $w, e, \delta$, and $F$, there always exists an equilibrium such that $w_{L}^{\sigma}<w_{R}^{\sigma}$, though this equilibrium need not be IE. Moreover, when $e_{L} \geq e_{R}$, all equilibria have this property. ${ }^{15}$ However, when $e_{L}<e_{R}$, there always exists an $F$ for which the game admits an IE with $w_{L}^{\sigma}>w_{R}^{\sigma}$. In such equilibria, $w_{L}^{\sigma}+e_{L}^{\sigma}<w_{R}^{\sigma}+e_{R}^{\sigma}$, so $R$ behaves in a more (less) interventionist way than $L$ with the efficient (inefficient) instrument. The intuition for such equilibria is as follows. If $e_{R}$ is sufficiently large, then $R$ strongly prefers intervention $p$ to $q$. But when the status quo is $n$ and $\theta$ is sufficiently large, $R$ approves a proposal $q$ by $L$ if such a proposal is made. To hedge against such a possibility, $R$ 's best response involves a bias against $n$ when $p$ is the status quo; that is, $R$ blocks repealing $p$ even for some states for which her flow-payoff from $n$ is higher. If this bias is large enough, we may have $w_{R}^{\sigma}<w_{L}^{\sigma}$, which means that $p$ stays in place for a larger set of states than $L$ would like. Anticipating $R$ 's bias and the resulting inertia under status quo $p, L$ proposes to intervene with $q$ rather than with $p$ when the status quo is $n$, rationalizing $R$ 's best response bias. ${ }^{16}$

\subsection{Use of inefficient instruments in equilibrium}

In this section, we discuss how the types of equilibria and the equilibrium parameters $\left(w^{\sigma}, e^{\sigma}\right)$ vary with the primitives of the model. To understand the discussion below, recall that from Proposition 2, an IE requires $e_{j}^{\sigma} \leq 0$ for some player $j$, where $e_{j}^{\sigma}$ is $j$ 's continuation payoff difference from a given period $t$ onward from implementing $p$ instead of $q$ in $t$, given continuation play $\sigma$. Policy $p$ yields a greater flow-payoff than $q$ in $t$, but status quo $p$ and $q$ may also induce different continuation policy paths in $t+1$. As illustrated in Figures 1 and 2, in $t+1$, either status quo $p$ and $q$ are both repealed (for $\theta$ small enough), in which case they lead to the same continuation path, or they both stay in place (for $\theta$ large enough), in which case players keep on getting a greater flow-payoff from $p$ than from $q$, or status quo $p$ stays in place whereas $q$ is repealed (for intermediate values of $\theta$ ), in which case the player $j$ who wants to repeal $p$ in such states gets a

\footnotetext{
${ }^{15}$ The proof that $e_{L} \geq e_{R}$ implies that in any equilibrium $\sigma, w_{L}^{\sigma}<w_{R}^{\sigma}$ follows directly from (24) in Lemma 4 in the Appendix. The proof that there always exists an equilibrium such that $w_{L}^{\sigma}<w_{R}^{\sigma}$ can be found in Proposition 5 in the working paper version of this paper available on the authors' websites.

${ }^{16}$ In the Appendix (see the example in Appendix A.4), we consider an environment in which $w_{L}=w_{R}$ and $e_{L}<e_{R}$, and we construct an IE $\sigma$ such that $w_{R}^{\sigma}<w_{L}^{\sigma}+e_{L}^{\sigma}<w_{R}^{\sigma}+e_{R}^{\sigma}<w_{R}^{\sigma}$. By continuity, for some $w_{L}<w_{R}$, there exists a nearby IE whose continuation payoff parameters satisfy the same inequality, which formally proves that there exists an IE in which the more interventionist player behaves as the least interventionist in equilibrium.
} 
greater continuation payoff from status quo $q$. Thus, for $e_{j}^{\sigma}$ to be negative, the third case must be sufficiently likely and beneficial to $j$, relative to the inefficiency of $q$.

We first discuss how the existence of IE depends on the degree of players' polarization over when to enact and repeal $p$, as captured by $\left(w_{L}, w_{R}\right)$. Fix $F$ and $\delta$, and, for simplicity, consider the case $e_{L}=e_{R}=e$ for some fixed $e>0$, that is, $q$ is equally inefficient for $L$ and $R$. In that case, as discussed in Section 4.1, in any equilibrium, $w_{L}^{\sigma}<w_{R}^{\sigma}$, so from Proposition 2, an IE requires $e_{R}^{\sigma} \leq 0$. In the extreme case of no polarization $w_{L}=w_{R}$, players do not expect any disagreement, and the inefficient instrument $q$ has no strategic value. As a result, the only equilibrium is an EE in which players behave as if they were playing a one-shot game, i.e., $w_{L}^{\sigma}=w_{R}^{\sigma}=w_{L}=w_{R}$ and $e_{L}^{\sigma}=e_{R}^{\sigma}=e$ (see part (iii) of Proposition 3). ${ }^{17}$ As players become more polarized (i.e., as $w_{R}$ increases and $w_{L}$ decreases), players' period preferences disagree over whether to repeal $p$ when $\theta \in\left[w_{L}, w_{R}\right]$. If $w_{R}-w_{L}$ is sufficiently small, these disagreement states are unlikely. Therefore, the greater repealability of $q$ does not compensate for its inefficiency, so $e_{R}^{\sigma}>0$ and all equilibria remain EE. However, each player is now biased in favor of the policy that gives her a greater payoff in the disagreement states, which implies that $w_{L}^{\sigma}<w_{L}<w_{R}<w_{R}^{\sigma}$. This means that the set of the states in which players disagree on the policy change is larger than in a one-shot game. As polarization increases further, players' equilibrium preferences over when to enact and repeal $p$ are more likely to disagree (i.e., $w_{L}^{\sigma}$ decreases and $w_{R}^{\sigma}$ increases further), so the greater repealability of $q$ becomes more attractive to $R$. As a result, $e_{R}^{\sigma}$ may become negative and an IE may arise. $^{18}$

As the conflict of interests becomes severe, i.e., as $\left(w_{L}, w_{R}\right) \rightarrow(-\infty,+\infty)$, the players are rarely aligned with respect to whether any intervention is warranted. Thus, both $p$ and $q$ are unlikely to be repealed, and the players perceive any decision as virtually permanent. As a result, $q$ loses its strategic value and all equilibria become EE again (see part (iii) of Proposition 3).

Now fix $F, \delta$, and players' polarization over the efficient intervention $\left(w_{L}, w_{R}\right)$, and consider the role of $\left(e_{L}, e_{R}\right)$. Given $e_{L}$, as $e_{R}$ increases, $q$ becomes more inefficient for player $R$. So if $e_{R}$ is sufficiently large, the guarantee of being able to repeal $q$ more easily tomorrow does not offset its inefficiency today and all equilibria are EE. Conversely, when $e_{R}$ is sufficiently small, the strategic value of $q$ always outweighs its inefficiency and all equilibria are IE (see part (i) of Proposition 3).

\footnotetext{
${ }^{17}$ Proposition 3 part (iii) does not assume $e_{L}=e_{R}$. In this more general case, it is still true that an EE always exists as $\left(w_{L}, w_{R}\right) \rightarrow(w, w)$. However, an IE may also exist (see the example in Appendix A.4 for a formal proof of that claim). Basically, when $e_{L} \neq e_{R}$, in some states, players' period preferences disagree when comparing $n$ and $q$, and this sincere disagreement between $n$ and $q$ can generate a strategic disagreement between $n$ and $p$ as well, even though $w_{L}=w_{R}$. To understand how such equilibria can be sustained, note that the player with the greater $e_{k}$, say $L$ for concreteness, may be concerned that under status quo $n$, for $\theta$ large enough, $R$ will propose $q$ and $L$ will have no choice but to accept it. To avoid this situation, $L$ becomes biased against $n$ and prefers to leave $p$ in place for a greater set of sets than $R$ would like. To avoid this inertia of status quo $p, R$ prefers to intervene with $q$ rather than with $p$, rationalizing $L$ 's initial concern.

${ }^{18}$ EE may disappear or may coexist, depending on the parameters. See the discussion under Proposition 5 or the example in Appendix A.4 for other examples in which IE and EE may coexist.
} 
Similarly, increasing $e_{L}$, given $e_{R}$, makes $q$ more inefficient for player $L$. In this case, however, there are two countervailing effects. On one hand, $L$ approves the repeal of $q$ in more states, which increases the strategic value of $q$ for $R$. On the other hand, $L$ approves proposal $q$ in fewer states. Regardless of the value of $e_{L}$, however, for $\theta$ large enough, $L$ prefers implementing $q$ to $n$, and $R$ can be sure $q$ is accepted and implemented on the equilibrium path. Intuitively then $R$ will suggest $q$ as long as she does not find it too inefficient. Hence, if for some parameters all equilibria are IE, then all equilibria remain IE as $q$ becomes more inefficient for player $L$ (see part (ii) of Proposition 3). This discussion implies that the inefficient policy instrument will be used when it is sufficiently costly for player $L$ and not too (in relative terms) costly for player $R$.

The following proposition formalizes the main claims above.

Proposition 3. Fix $\delta \in(0,1)$, the recognition probability functions $b_{L}$ and $b_{R}$, and the c.d.f. F. Then the following statements hold:

(i) For any $w_{L}, w_{R}$, and $e_{L}$, there exists $\bar{e}_{R} \in(0,+\infty)$ such that all equilibria are IE for all $e_{R}<\bar{e}_{R}$, and for all $e_{R}>\bar{e}_{R}$, there exists EE.

(ii) For any $w_{L}, w_{R}$, and $e_{R}$, if all equilibria are IE for some $e_{L}$, they are all IE for $e_{L}^{\prime}>e_{L}$

(iii) For any $\left(e_{L}, e_{R}\right)$ and any average ideology $\left(w_{L}+w_{R}\right) / 2$, as $\left(w_{R}-w_{L}\right) \rightarrow+\infty$, then all equilibria are $E E$, and as $\left(w_{R}-w_{L}\right) \rightarrow 0$, there exists an $E E$.

The last claim of Proposition 3 sheds light on how the use of inefficient instruments may relate to the nature of the political system. The existence of checks and balances in a multi-member political system reflects a demand for some degree of "consensus" for policy change. Examples include supermajority voting rules, as mentioned earlier, or agreement between the median voter in a legislature subject to simple majority rule and the president. This sort of consensus in our model is approximated by the level of polarization, $\left(w_{R}-w_{L}\right)$ : the larger is this distance, the greater is the implicit level of consensus required for policy change (see Dziuda and Loeper 2018 and Krehbiel 1998). Consequently, to the extent that more checks and balances are associated with demands for more consensus, Proposition 3 implies that inefficient instruments are likely to be used in political systems with moderate polarization and moderate degrees of checks and balances.

We emphasize, however, that the above statements hold for fixed $\delta$ and $F$. So a particular degree of polarization over efficient intervention may lead to EE for some patience and distribution of states of nature, but may lead to IE for other values of these primitives. The following proposition shows that for any payoff parameters $\left(w_{L}, w_{R}, e_{L}, e_{R}\right)$, all equilibria are IE for some patience and distribution of the state. In particular, no matter how inefficient the policy instrument $q$ is, all equilibria may be IE.

Proposition 4. Let $F$ be a c.d.f. with mean 0 and variance 1 , and let $\left(F_{m, d}\right)_{m \in \mathbb{R}, d>0}$ denote the corresponding location-scale family of c.d.f., where, for all $m \in \mathbb{R}$ and $d>0$, 
$F_{m, d}(\theta) \equiv F\left(\frac{\theta-m}{d}\right)$. Then for any $\left(w_{L}, w_{R}, e_{L}, e_{R}\right)$, there exists $\bar{d}>0, \underline{\delta}<\bar{\delta}$, and $\underline{m}<\bar{m}$ such that for all $\delta \in[\underline{\delta}, \bar{\delta}], m \in[\underline{m}, \bar{m}]$, and $d \in(0, \bar{d}]$, all equilibria of $\Gamma$ are IE for the c.d.f. $F_{m, d}$.

The proposition is predicated on the observation that two things must be true for all equilibria to be IE. Players must frequently disagree over whether $n$ or $p$ is a better policy, but they must frequently agree over whether $n$ or $q$ is a better policy. To guarantee the former, in the proof, we select $[\underline{m}, \bar{m}]$ within the set of states $\left(w_{L}, w_{R}\right)$ in which $L$ wants to maintain $p$ whereas $R$ wants to repeal it, and we select $\bar{d}$ small enough so that most of the probability mass is concentrated around the mean $m \in[\underline{m}, \bar{m}]$ of $F_{m, d}$. To guarantee the latter, we select $\bar{m}$ to the left of $w_{L}+e_{L}$ and $w_{R}+e_{R}$, so that in the most frequent states close to $m$, both players prefer no intervention $n$ to the inefficient intervention $q$. We then choose the minimum level of patience $\underline{\delta}$ such that the greater repealability of $q$ outweighs its inefficiency for $R$ and $R$ prefers to intervene with $q$ rather than with $p$. However, if players are too patient, an EE may also exist. The reason is that in states close to $m$, even though $L$ gets a greater flow-payoff from $n$ than from $q$, under status quo $q, L$ may credibly commit to accept and propose only $p$, in which case $R$ has no choice but to accept and propose only $p$ because $R$ prefers a permanent $p$ to a permanent $q$. Given these expectations, $R$ will prefer not to use $q$ in the first place. Thus, for all equilibria to be IE, we need to impose an upper bound $\bar{\delta}$ on players' patience. It is worth emphasizing, however, that the conditions identified in the proof of Proposition 4 are by no means necessary for the existence of IE. ${ }^{19}$

\subsection{The value of inefficient instruments}

In reality, the menu of available policy instruments is often endogenous. For example, if environmental policy is chosen at the local level or by a regulatory body, then the instruments that are available may be restricted by the federal government and, in such cases, it is not at all clear whether an inefficient policy instrument would, or should, be made available to decision makers. As argued in Section 2, there is no social welfare gain to be had from the existence of $q$ in a static environment; the question is whether this holds in dynamic settings. The answer is "yes": allowing for an inefficient policy instrument can lead to Pareto superior equilibria in the dynamic game. Although a more general existence result is available (Austen-Smith et al. 2019, Proposition 5), we establish the efficiency claim here with a simple two-state example. Additionally, the example provides insight on the environments in which $q$ can be expected to be welfare improving.

Let $\Gamma(n, p, q)$ denote the original game, let $\Gamma(n, p)$ denote the game in which the inefficient instrument is unavailable, and let $\Gamma(n, q)$ denote the game in which the efficient instrument is unavailable. Say that one equilibrium is Pareto superior to another if at the beginning of the game, for any realization of the initial state, both players get a strictly greater continuation payoff in the former. Then we have the following result.

\footnotetext{
${ }^{19} \mathrm{~A}$ similar result for the exclusive existence of EE holds. For instance, for any $m$ and $\delta$, one can show that as $d$ becomes sufficiently large, that is, as extreme states become sufficiently likely, all equilibria are EE.
} 
Proposition 5. Suppose there exist $\underline{\theta}<\bar{\theta}$ and $\pi \in(0,1)$ such that in every period $t$, with probability $1-\pi, \theta(t)=\underline{\theta}$, and with probability $\pi, \theta(t)=\bar{\theta}$.

(i) There exists an equilibrium of $\Gamma(n, p, q)$ that is Pareto superior to any equilibrium of $\Gamma(n, p)$ if and only if

$$
\begin{aligned}
w_{L} & <\underline{\theta} \leq \min \left\{w_{i}+e_{i}\right\} \leq \max \left\{w_{i}+e_{i}\right\}<\bar{\theta} \\
\delta(1-\pi)\left(w_{R}-\underline{\theta}\right) & >(1-\delta(1-\pi))\left(\bar{\theta}-w_{R}\right) .
\end{aligned}
$$

(ii) Furthermore, all equilibria of $\Gamma(n, p, q)$ are Pareto superior to all equilibria of $\Gamma(n, p)$ if and only if (3), (4), and the following inequality hold:

$$
e_{L}>\frac{\delta \pi}{1-\delta}\left(\bar{\theta}-w_{L}\right)+\frac{1-\delta \pi}{1-\delta}\left(\underline{\theta}-w_{L}\right) .
$$

(iii) For any $\left(w_{L}, w_{R}, e_{L}, e_{R}\right)$, there exists $\delta$ and $F$-i.e., a triple $(\underline{\theta}, \bar{\theta}, \pi)$-such that all equilibria of $\Gamma(n, p, q)$ are Pareto superior to all equilibria of $\Gamma(n, p)$.

Parts (i), (ii), and (iii) above hold unchanged if we replace $\Gamma(n, p, q)$ by $\Gamma(n, q)$.

Proposition 5 part (i) characterizes the conditions under which adding $q$ can make both players strictly better off. To understand the intuition for, and implications of, these conditions, note first that condition (3) is essentially equivalent to the condition (1) in the two-period example of Section 2 and has a similar interpretation as in the two-period setup. ${ }^{20}$ Specifically, the last three inequalities in (3) mean that when choosing between $n$ and $q$, players' static preferences are the same, so in the game $\Gamma(n, q)$, there exists an equilibrium in which players agree to implement $n$ in state $\underline{\theta}$ and $q$ in state $\bar{\theta}$. The first inequality in (3) means that $p$ is $L$ 's most preferred policy in either state, so status quo $p$ is never repealed. Hence, in the game $\Gamma(n, p)$, if $n$ is the status quo and $R$ 's preference for $n$ over $p$ in the low state $\underline{\theta}$ is stronger than her preference for $p$ over $n$ in the high state $\bar{\theta}, R$ will not approve intervening with $p$ and in any equilibrium, players will stay at $n$ forever. This is assured by condition (4), and in that case, both players are strictly worse off in this nonintervention equilibrium path than in the equilibrium path of $\Gamma(n, q)$, described above.

Now consider the game $\Gamma(n, p, q)$. Since, under (4), $R$ does not approve $p$, adding $p$ to the game leaves the incentives underlying the equilibria of $\Gamma(n, q)$ unchanged. Agreeing to implement $n$ in state $\underline{\theta}$ and $q$ in state $\bar{\theta}$, therefore, is also an equilibrium of $\Gamma(n, p, q)$, and both players strictly prefer it to the unique equilibrium path of $\Gamma(n, p)$. Hence, for the inefficient instrument to be beneficial in some equilibrium, players' degree of polarization must be sufficiently large so that they disagree sufficiently often on when to repeal $p$, but not so large that they agree sufficiently often on when to repeal $q$.

Part (ii) shows that the additional condition (5) is required to ensure both players are strictly better off in all equilibria of $\Gamma(n, p, q)$ relative to $\Gamma(n, p)$. The reason is that IE

\footnotetext{
${ }^{20}$ The only difference between conditions (1) and (3) is that the former requires $\underline{\theta}<w_{R}$, but this extra condition is implied by (4).
} 
and EE may coexist in $\Gamma(n, p, q)$ : staying with $n$ indefinitely, as in the unique equilibrium path of $\Gamma(n, p)$, may remain an equilibrium in $\Gamma(n, p, q)$, leaving $L$ and $R$ indifferent between the two games. To see how this can occur, suppose that $L$ threatens to approve only $p$ if ever $q$ is implemented. If such a threat is credible, $R$ never approves $q$ and no intervention is ever implemented. Condition (5) guarantees that no such threat is credible: when $e_{L}$ is sufficiently large, $L$ prefers to accept $n$ rather than stay at $q$ in state $\underline{\theta}$. Thus, to guarantee that both players benefit unambiguously from the availability of $q, q$ must be sufficiently inefficient for the less interventionist players.

It is worth noting how the conditions of Proposition 5 depend on $\pi$ and $\delta$. From (4) and (5), the set of $w_{R}, w_{L}$, and $e_{L}$ that satisfies these conditions expands as $\pi$ falls. Hence, both players are more likely to benefit from $q$ if the state in which they disagree over intervening with the efficient policy becomes more likely. In addition, as $\delta$ increases and players become more patient, the set of $w_{R}$ satisfying (4) increases and a welfare improving IE equilibrium, therefore, becomes more likely. However, the right-hand side of (5) first decreases and then increases with $\delta$. Hence, to benefit unambiguously from the presence of $q$, players should be sufficiently, but not excessively, patient. ${ }^{21}$ Part (iii) further states that for any payoff parameters, the availability of $q$ unambiguously leads to Pareto better equilibria for some degree of patience and some specification of $F$.

The main message of Proposition 5 remains unchanged even if we allow for nonMarkov strategies. Indeed, if conditions (3) and (4) are satisfied, then there exists a subgame-perfect Nash equilibrium (SPNE) of $\Gamma(n, p, q)$ that Pareto dominates any SPNE of $\Gamma(n, p)$. To see this, simply note that under condition (3), having policy $p$ forever gives a strictly greater payoff to $L$ than any other policy path. So in any SPNE of $\Gamma(n, p), p$ stays in place forever once it becomes the status quo. Thus, condition (4) implies than in any SPNE of $\Gamma(n, p)$, the initial status quo $n$ stays in place forever. The Markov equilibrium $\sigma$ of $\Gamma(n, p, q)$ in which players agree to implement $n$ in state $\underline{\theta}$ and $q$ in state $\bar{\theta}$ is a fortiori a SPNE, and from what precedes, it Pareto dominates any SPNE of $\Gamma(n, p)$. Note further that under conditions (3) and (4), allowing for non-Markov strategies does not lead to more efficient outcomes. Since under (3), in any SPNE, $p$ stays in place forever once implemented, the path of $\sigma$ is the most preferred path of $R$ among all such paths, so $\sigma$ is $R$ 's most preferred SPNE. Outside of the environment of Proposition 5, allowing for non-Markov strategies may expand the set of equilibria to include more efficient equilibria, but the main qualitative message of the paper should survive, namely, that $q$ may be used in equilibrium and ruling out $q$, either by selecting a different equilibrium or by allowing players to commit not to use $q$, can make them worse off.

\section{Volatility AND PERSistent STATES}

Until now, states, and thereby players' state-contingent preferences, have been assumed i.i.d. draws over time. In many applications, however, there may be periods of relative

\footnotetext{
${ }^{21}$ The non-monotonic impact of $\delta$ on the value of inefficient instruments is not an artifact of the twostate distributions considered in Proposition 5. Condition (3) guarantees that players are in full ideological agreement when choosing between the inefficient instrument $q$ and no intervention $n$. This makes $q$ particularly attractive. If the distribution of the state has full support, sufficiently patient players may introduce an inefficient gridlock also to the choice between $n$ and $q$. As a result, unlike in the two-state case, $q$ may not strictly improve both players' payoffs if players are sufficiently patient and, further, an IE may not exist.
} 
stability when players do not expect to change their positions, other things equal, and there may also be periods in which new information about the desirability of an intervention arrives frequently, resulting in frequent revisions of the relevant policy preferences. ${ }^{22}$ Our main argument, therefore, that one reason for rational legislators to reject an efficient policy in favor of an inefficient policy is because inefficient policies are easier to repeal, is attenuated to the extent that it depends essentially on the i.i.d. assumption. Consequently, we extend the argument to a more general environment in which the i.i.d. assumption is relaxed to permit serial correlation.

To capture the possibility that states may persist across periods in an analytically tractable way and to allow for players' expectations regarding the persistence of the current state to vary over time, consider, for every period $t$, a tuple $(\theta(t), v(t)) \in \mathbb{R} \times[0,1]$. Assume that the evolution of such tuples across periods satisfies the following transition property: for all $t$,

$$
(\theta(t+1), v(t+1))= \begin{cases}(\theta(t), v(t)) & \text { with probability } 1-v(t) \\ (\theta(t+1), v(t+1)) \sim H & \text { with probability } v(t),\end{cases}
$$

where $H$ is some joint c.d.f. As before, $\theta_{t}$ is the underlying policy-relevant state. We interpret the additional variable $v(t)$ as a measure of volatility of the current policyrelevant state $\theta(t)$ or, equivalently, of players' period $t$ expectations over $\theta(t+1)$. In each period $t$, the state $\theta(t)$ persists into period $t+1$ with probability $(1-v(t))$ and, for simplicity, assume the volatility $v(t)$ also persists into period $t+1$; with probability $v(t)$, $\theta(t+1)$ and $v(t+1)$ are drawn according to the joint c.d.f. $H$. Thus, the volatility of future state-contingent policy preferences is redrawn if and only if the state-contingent policy preferences are redrawn.

Note that the evolution of the state collapses to the basic i.i.d. model if $v(t) \equiv 1$ for all $t$. Similarly, $v(t) \equiv 0$ for all $t$ implies preferences never change, while $v(t) \equiv v \in(0,1)$ for all $t$ implies the degree of volatility is fixed. In the following proposition, however, we consider the general model described above in which the volatility evolves over time and $v(t)$ is redrawn every period according to $H$.

Proposition 6. In any equilibrium $\sigma$, there exists $\bar{v} \in(0,1]$ such that $q$ is not implemented when the realization of volatility is $v<\bar{v}$, but $q$ is implemented with positive probability under status quo $n$ when the realization of volatility is $v>\bar{v}$. Moreover, for any $\left(w_{L}, w_{R}, e_{L}, e_{R}\right)$, for all $\delta$ sufficiently close to 1 , there exists distribution $H$ with full support on $\mathbb{R} \times[0,1]$ such that, for all equilibria, $\bar{v}<1$.

Proposition 6 states that $q$ is implemented on the equilibrium path only in sufficiently volatile economic environments. Intuitively, when players expect the current

\footnotetext{
${ }^{22}$ A painful example is the U.S. Congressional response to the 2008 fiscal collapse. For some years before 2008, the U.S. economy was growing strongly and atypical Congressional economic interventions were minimal. The fall of Lehman Brothers and the subsequent turmoil in much of the global economy led to serious Congressional disagreement regarding the appropriate level and duration of any extraordinary intervention, from whether to bail out banks or the car industry to extensions of unemployment and welfare benefits.
} 
state to be lasting $(v(t) \leq \bar{v})$, strategic concerns regarding the possibility of conflict over repealing today's intervention tomorrow, say, are muted and any intervention is efficient. When the economic environment is expected to be transient, however $(v(t)>\bar{v})$, today's choice is likely to need revision in the next period, making salient exactly the sorts of strategic consideration underlying the use of inefficient interventions. ${ }^{23}$

\section{Conclusion}

The continued and widespread use of inefficient policy interventions in more-or-less democratic political systems is a puzzle. For example, while economists uniformly recommend regulating emissions with Pigou taxes, technology and quantity controls are the most adopted instruments in reality. Why would rational politicians agree on the use of Pareto-dominated policy instruments? In our model, an inefficient policy intervention may arise even when there is no salient legislative history or vested interest. Rather, an inefficient policy may be chosen precisely because it is inefficient and the environment is expected to change in the future. From this perspective, the puzzle alluded to above can be understood without pointing to informational asymmetries, interest group influence, or differential distributional implications of alternative policy instruments among the electorate at large.

With a heterogeneous legislature and multiple veto players, inefficient interventions are politically easier than efficient interventions to repeal in dynamic environments subject to policy-relevant stochastic shocks. In addition, since inefficient interventions are easier to repeal, heterogeneous veto players, differentiated only by the threshold shocks beyond which they judge some policy intervention to be warranted, can be more willing to agree on responding to a sufficiently severe downside shock with an inefficient instrument. As a consequence, inefficient interventions are more likely to be used in (moderately) polarized political environments and for issues where the fundamentals are subject to change over time.

\section{APPENDiX}

\section{A.1 Notations and preliminary lemmas}

Notation 1. Throughout the Appendix, the term "strategy" refers to a Markov strategy and the term "equilibrium" refers to a Markov perfect equilibrium, and we allow mixed strategies (in the behavioral sense). That is, we assume that in each period $t$, each player

\footnotetext{
${ }^{23}$ Note that in the special case of the model in which $v(t) \equiv v^{o}$ for all $t$ for some $v^{o}$, Proposition 6 is actually vacuous, as it simply states that either the equilibrium is IE (if $v^{o}>\bar{v}$ ) or is EE (if $v^{o}<\bar{v}$ ), but it does not deliver any comparative statics with respect to $v^{o}$ and $\bar{v}$ depends on $v^{o}$. Such comparative statics are in general ambiguous. To see why, note that as $v^{o}$ increases, today's preferences are more likely to be transient, so players care less about the flow-payoff they get from today's policy relative to which policy gives them a better bargaining position tomorrow. This makes the less interventionist player more willing to intervene with $q$ than with $p$. However, an increase in $v^{o}$ also makes tomorrow's preferences more likely to be transient. This makes the more interventionist player less willing to repeal any intervention tomorrow. The greater inertia under $q$ or $p$ has an ambiguous effect on the willingness of the less interventionist player to use $q$.
} 
$i$ privately observes the realization of a payoff-irrelevant random variable $\rho_{i}(t)$ and can condition her action in period $t$ only on payoff-relevant variables and on $\rho_{i}(t)$, where $\left\{\rho_{L}(t): t \in \mathbb{N}\right\}$ and $\left\{\rho_{R}(t): t \in \mathbb{N}\right\}$ are i.i.d. and independent of each other.

For a given strategy profile $\sigma$, the policy outcome in period $t \in \mathbb{N}$ with status quo $s(t) \in\{n, p, q\}$ can depend on the following exogenous stochastic factors: the state of nature $\theta(t)$, the identity $p(t)$ of the proposer recognized in period $t$, and the realization of players' private randomization devices $\left(\rho_{L}(t), \rho_{R}(t)\right)$. Let $v(t) \equiv$ $\left(\theta(t), p(t), \rho_{L}(t), \rho_{R}(t)\right)$ denote the random variable that encodes this information. We refer to $v(t)$ as the state of the world in period $t$. Let $Y$ denote the set of possible states of the world. Note that $\{v(t): t \in \mathbb{N}\}$ is i.i.d. Let $\mu$ denote its probability distribution. For any state of the world $v \in Y, \theta(v)$ denotes the corresponding realization of the state of nature. For all $s, x \in\{n, p, q\}, Y^{\sigma}(s, x)$ denotes the set of realizations of the state of the world for which status quo $s$ leads to outcome $x$ if players play $\sigma$.

Let $V_{i}^{\sigma}(\theta, x)$ denote the expected discounted payoff for player $i \in\{L, R\}$ from implementing policy $x \in\{n, p, q\}$ in period 0 conditional on $\theta(0)=\theta$ and on players playing $\sigma$ from period 1 onward.

Lemma 2 characterizes the continuation payoff parameters introduced in Lemma 1 (equilibrium existence will be established latter on). Lemma 2 also derives some necessary conditions on $\left(w^{\sigma}, e^{\sigma}\right)$ for $\sigma$ to be an equilibrium. Lemma 3 provides necessary and sufficient conditions on $\left(w^{\sigma}, e^{\sigma}\right)$ for $\sigma$ to be an EE. These conditions allow us to derive conditions on the primitive parameters under which no EE exists and, thus, under which all equilibria are IE (see the proof of Proposition 3).

Definition 2. Let $\left(w^{c}, e^{c}\right) \in \mathbb{R}^{4}$. A strategy profile $\sigma$ is subgame perfect for the continuation payoff parameters $\left(w^{c}, e^{c}\right) \in \mathbb{R}^{4}$ if $\sigma$ is a subgame-perfect equilibrium of the game in which players play a single period of $\Gamma$ with payoffs $V$ defined by, for all $\theta \in \mathbb{R}$, $V_{i}(\theta, p)-V_{i}(\theta, n)=\theta-w_{i}^{c}$ and $V_{i}(\theta, p)-V_{i}(\theta, q)=e_{i}^{c}$.

\section{Lemma 2. Let $\sigma$ be a strategy profile.}

(A) Recall the definition of $V^{\sigma}$ from Notation 1. There exist $w_{i}^{\sigma} \in \mathbb{R}$ and $e_{i}^{\sigma} \in \mathbb{R}$ such that, for all $\theta \in \mathbb{R}$,

$$
\begin{aligned}
& V_{i}^{\sigma}(\theta, p)-V_{i}^{\sigma}(\theta, n)=\theta-w_{i}^{\sigma} \\
& V_{i}^{\sigma}(\theta, p)-V_{i}^{\sigma}(\theta, q)=e_{i}^{\sigma} \\
& V_{i}^{\sigma}(\theta, q)-V_{i}^{\sigma}(\theta, n)=\theta-w_{i}^{\sigma}-e_{i}^{\sigma} .
\end{aligned}
$$

Expression $\frac{w_{i}^{\sigma}-w_{i}}{\delta}\left(\frac{e_{i}^{\sigma}-e_{i}}{\delta}\right)$ is equal to the expected payoff gain for player $i$ of having initial status quo $n(p)$ instead of $p(q)$ in the game $\Gamma$ given continuation play $\sigma$.

(B) Let $w_{i}^{\sigma}$ and $e_{i}^{\sigma}$ be the continuation payoff parameters of $\sigma$, as characterized in (6). Then $\sigma$ is an equilibrium if and only if $\sigma$ is subgame perfect for $w_{i}^{\sigma}$ and $e_{i}^{\sigma}$ in the sense of Definition 2. 
(C) If $\sigma$ is an equilibrium, then

$$
\begin{aligned}
\frac{w_{i}^{\sigma}-w_{i}}{\delta}= & \int_{\Upsilon^{\sigma}(p, p) \cap \Upsilon^{\sigma}(n, n)}\left(w_{i}^{\sigma}-\theta(v)\right) d \mu(v)-\int_{\Upsilon^{\sigma}(p, p) \cap \Upsilon^{\sigma}(n, q)} e_{i}^{\sigma} d \mu(v) \\
\frac{e_{i}^{\sigma}-e_{i}}{\delta}= & \int_{\Upsilon^{\sigma}(p, p) \cap \Upsilon^{\sigma}(q, q)} e_{i}^{\sigma} d \mu(v)+\int_{\Upsilon^{\sigma}(p, p) \cap \Upsilon^{\sigma}(q, n)}\left(\theta(v)-w_{i}^{\sigma}\right) d \mu(v) \\
& +\int_{\Upsilon^{\sigma}(p, n) \cap \Upsilon^{\sigma}(q, q)}\left(w_{i}^{\sigma}+e_{i}^{\sigma}-\theta(v)\right) d \mu(v) .
\end{aligned}
$$

Proof. By definition, $V_{i}^{\sigma}(\theta, p)-V_{i}^{\sigma}(\theta, n)$ is the sum of the flow-payoff gain from implementing $p$ instead of $n$ in $t=0$ when $\theta(0)=\theta$ and $\delta$ times the continuation payoff gain from period 1 onward from having $s(1)=p$ instead of $s(1)=n$, given continuation play $\sigma$ in $t \geq 1$. So using Notation 1 , we can write

$$
\begin{aligned}
& V_{i}^{\sigma}(\theta, p)-V_{i}^{\sigma}(\theta, n) \\
& \quad=\theta-w_{i}+\delta \sum_{x, y \in\{n, p, q\}} \int_{\mathcal{Y}^{\sigma}(p, x) \cap \Upsilon^{\sigma}(n, y)}\left(V_{i}^{\sigma}(\theta(v), x)-V_{i}^{\sigma}(\theta(v), y)\right) d \mu(v) .
\end{aligned}
$$

Therefore, to prove the first line of (6), it suffices to set

$$
w_{i}^{\sigma} \equiv w_{i}-\delta \sum_{x, y \in\{n, p, q\}} \int_{\Upsilon^{\sigma}(n, x) \cap Y^{\sigma}(p, y)}\left(V_{i}^{\sigma}(\theta(v), x)-V_{i}^{\sigma}(\theta(v), y)\right) d \mu(v) .
$$

An analogous reasoning on the continuation payoff gain from implementing $p$ instead of $q$ proves the second line of (6) with

$$
e_{i}^{\sigma} \equiv e_{i}+\delta \sum_{x, y \in\{n, p, q\}} \int_{Y^{\sigma}(p, x) \cap \Upsilon^{\sigma}(q, y)}\left(V_{i}^{\sigma}(\theta(v), x)-V_{i}^{\sigma}(\theta(v), y)\right) d \mu(v) .
$$

The third line of (6) is simply the sum of the first two.

Using Definition 2 and part A, part B simply restates the fact that $\sigma$ is a Markovperfect equilibrium if and only if in any period $t, \sigma$ prescribes actions that are subgame perfect given continuation play $\sigma$ from $t+1$ onward.

To prove part C, let $\sigma$ be an equilibrium. Using (10), we get $\frac{e_{i}^{\sigma}-e_{i}}{\delta}=$ $\sum_{x, y \in\{n, p, q\}} e_{i}^{\sigma}(x, y)$, where

$$
e_{i}^{\sigma}(x, y) \equiv \int_{\Upsilon^{\sigma}(p, x) \cap Y^{\sigma}(q, y)}\left(V_{i}^{\sigma}(\theta(v), x)-V_{i}^{\sigma}(\theta(v), y)\right) d \mu(v) .
$$

For $x=y$ clearly $e_{i}^{\sigma}(x, y)=0$. Consider next $(x, y)=(q, n)$, that is, status quo $p$ is replaced by $q$ and status quo $q$ is replaced by $n$. Since $\sigma$ is an equilibrium, for all $v \in \Upsilon^{\sigma}(p, q) \cap \Upsilon^{\sigma}(q, n)$, both players must weakly prefer to implement $q$ to $p$ and $n$ to $q$ in period 1, given continuation play $\sigma$. Moreover, one of the players must be indifferent between implementing $n$ and $q$, because if both players strictly preferred to implement $n$ to $q$ in state $v$, then under status quo $p$, either veto player would accept 
$n$, so proposing $n$ instead of $q$ in that period would be a profitable deviation for the proposer. Therefore, for all $v \in \Upsilon^{\sigma}(p, q) \cap \Upsilon^{\sigma}(q, n), V_{i}^{\sigma}(\theta(v), q)-V_{i}^{\sigma}(\theta(v), n)=0$ for some player $i$, and using (6), this implies $\theta(v)-w_{i}^{\sigma}-e_{i}^{\sigma}=0$. But that equality is satisfied only in states of the world for which the corresponding realization of the state of nature is $\theta(v)=w_{i}^{\sigma}+e_{i}^{\sigma}$, but since $F$ is continuous, this happens with probability 0 , so $\mu\left(\Upsilon^{\sigma}(p, q) \cap \Upsilon^{\sigma}(q, n)\right)=0$ and so $e_{i}^{\sigma}(q, n)=0$. In the case $(x, y)=(p, n)$, an analogous reasoning implies that $\mu\left(\Upsilon^{\sigma}(q, p) \cap \Upsilon^{\sigma}(p, n)\right)=0$, so $e_{i}^{\sigma}(p, n)=0$. Consider now $(x, y)=(q, p)$, that is, status quo $p$ is replaced by $q$ and vice versa. Since $\sigma$ is an equilibrium, for all $v \in \Upsilon^{\sigma}(p, q) \cap \Upsilon^{\sigma}(q, p), V_{i}^{\sigma}(\theta(v), p)=V_{i}^{\sigma}(\theta(v), q)$ for both players, so from (11), $e_{i}^{\sigma}(q, p)=0$. The only remaining cases are $(x, y)$ equal to $(p, q),(p, n)$, and $(n, q)$, so (10) becomes

$$
\begin{aligned}
\frac{e_{i}^{\sigma}-e_{i}}{\delta}= & \int_{\Upsilon^{\sigma}(p, p) \cap \Upsilon^{\sigma}(q, q)}\left(V_{i}^{\sigma}(\theta(v), p)-V_{i}^{\sigma}(\theta(v), q)\right) d \mu(v) \\
& +\int_{\Upsilon^{\sigma}(p, p) \cap \Upsilon^{\sigma}(q, n)}\left(V_{i}^{\sigma}(\theta(v), p)-V_{i}^{\sigma}(\theta(v), n)\right) d \mu(v) \\
& +\int_{\Upsilon^{\sigma}(p, n) \cap \Upsilon^{\sigma}(q, q)}\left(V_{i}^{\sigma}(\theta(v), n)-V_{i}^{\sigma}(\theta(v), q)\right) d \mu(v) .
\end{aligned}
$$

Substituting (6) into the above expression, we obtain (8).

The proof of (7) follows a similar logic. Using (9), we get that $\frac{w_{i}^{\sigma}-w_{i}}{\delta}=$ $\sum_{x, y \in\{n, p, q\}} w_{i}^{\sigma}(x, y)$, where

$$
w_{i}^{\sigma}(x, y) \equiv \int_{\Upsilon^{\sigma}(p, x) \cap \Upsilon^{\sigma}(n, y)}\left(V_{i}^{\sigma}(\theta(v), x)-V_{i}^{\sigma}(\theta(v), y)\right) d \mu(v) .
$$

Using the same steps as those used to prove $\mu\left(\Upsilon^{\sigma}(p, q) \cap \Upsilon^{\sigma}(q, n)\right)=0$, and reversing the role of $p$ and $q$, we obtain that $\mu\left(\Upsilon^{\sigma}(p, n) \cap \Upsilon^{\sigma}(n, q)\right)=0$, so $w_{i}^{\sigma}(n, q)=0$. An analogous reasoning implies that $\mu\left(\Upsilon^{\sigma}(p, q) \cap \Upsilon^{\sigma}(n, p)\right)=0$, so $w_{i}^{\sigma}(q, p)=0$. Moreover, for all $v \in \Upsilon^{\sigma}(p, n) \cap \Upsilon^{\sigma}(n, p)$, each player $i$ must be indifferent between implementing $n$ and $p$, so $\theta(v)-w_{i}^{\sigma}-e_{i}^{\sigma}=0$. Since $F$ is continuous, this implies that $\mu\left(\Upsilon^{\sigma}(p, n) \cap \Upsilon^{\sigma}(n, p)\right)=0$, so $w_{i}^{\sigma}(n, p)=0$. The only remaining cases left are $(x, y)$ equal to $(p, n),(p, q)$, and $(q, n)$. Equation (7) follows then from substituting these three cases into the right-hand side of (9) and using (6).

Lemma 3 (Necessary and sufficient conditions for EE). (A) Let $\sigma$ be an EE. Then $e_{L}^{\sigma}>0$, $e_{R}^{\sigma} \geq 0, w_{L}^{\sigma}<w_{L}<w_{R}<w_{R}^{\sigma}, \Upsilon^{\sigma}(q, q) \subseteq \Upsilon^{\sigma}(p, p)$, and for all $i \in\{L, R\}$,

$$
\frac{w_{i}^{\sigma}-w_{i}}{\delta}=\int_{w_{L}^{\sigma}}^{w_{R}^{\sigma}}\left(w_{i}^{\sigma}-\theta\right) d F(\theta) .
$$

(B) For any $\sigma$ that is EE, there exists $\sigma^{\prime}$ that is also EE and is such that players never propose $q$ under any status quo, $w^{\sigma^{\prime}}=w^{\sigma}$ and for all $i \in\{L, R\}$,

$$
\frac{e_{i}^{\sigma^{\prime}}-e_{i}}{\delta}=\int_{w_{L}^{\sigma}}^{\min \left\{w_{L}^{\sigma}+e_{L}^{\sigma^{\prime}}, w_{R}^{\sigma}\right\}} b_{R}(\theta, q)\left(\theta-w_{i}^{\sigma}\right) d F(\theta) .
$$


(C) Reciprocally, if there exists $\left(w^{*}, e^{*}\right) \in \mathbb{R}^{4}$ such that $e_{R}^{*} \geq 0$ and such that

$$
\begin{aligned}
\frac{w_{i}^{*}-w_{i}}{\delta} & =\int_{w_{L}^{*}}^{w_{R}^{*}}\left(w_{i}^{*}-\theta\right) d F(\theta) \\
\frac{e_{i}^{*}-e_{i}}{\delta} & =\int_{w_{L}^{*}}^{\min \left\{w_{L}^{*}+e_{L}^{*}, w_{R}^{*}\right\}} b_{R}(\theta, q)\left(\theta-w_{i}^{*}\right) d F(\theta),
\end{aligned}
$$

then there exists an EE $\sigma$ such that the corresponding continuation payoff parameters $\left(w^{\sigma}, e^{\sigma}\right)$ (as defined in Lemma 2A) satisfy $\left(w^{\sigma}, e^{\sigma}\right)=\left(w^{*}, e^{*}\right)$.

Proof. Part A. Let $\sigma$ be an arbitrary EE.

Step A1: $w_{L}^{\sigma}<w_{R}^{\sigma}$ and for all $i \in\{L, R\}$, we have

$$
\frac{w_{i}^{\sigma}-w_{i}}{\delta}=\int_{Y^{\sigma}(p, p) \cap \Upsilon^{\sigma}(n, n)}\left(w_{i}^{\sigma}-\theta(v)\right) d \mu(v) .
$$

Since $\sigma$ is an EE, $\mu\left(Y^{\sigma}(n, q)\right)=0$. Substituting the latter equality into (7), we obtain (16). Taking differences across players in (16) and solving for $w_{R}^{\sigma}-w_{L}^{\sigma}$, we obtain $w_{R}^{\sigma}-w_{L}^{\sigma}=$ $\frac{w_{R}-w_{L}}{1-\delta \mu\left(\Upsilon^{\sigma}(p, p) \cap Y^{\sigma}(n, n)\right)}>0$.

Step A2: $e_{L}^{\sigma} \geq 0$ and $e_{R}^{\sigma} \geq 0$. Note first that in any state $\theta>w_{k}^{\sigma}+e_{k}^{\sigma}$, from Lemma 2A, player $k$ strictly prefers implementing $q$ to $n$, given continuation play $\sigma$, and if $e_{k}^{\sigma}<0$, $k$ also prefers implementing $q$ to $p$. So if $e_{i}^{\sigma}<0$ for some $i \in\{L, R\}$, then since $\sigma$ is an equilibrium, in any period $t$ in which $s(t)=n, i$ is the proposer, and $\theta(t)>$ $\max _{k \in\{L, R\}}\left\{w_{k}^{\sigma}+e_{k}^{\sigma}\right\}$, the other player $j$ must accept proposal $q$, so $i$ must propose $q$, which contradicts $\sigma$ being an EE.

Step A3: Modulo a zero measure set, $\Upsilon^{\sigma}(p, p)=\left\{v \in \Upsilon: \theta(v)>w_{L}^{\sigma}\right\}$ and $\Upsilon^{\sigma}(n, n)=$ $\left\{v \in \mathcal{Y}: \theta(v)<w_{R}^{\sigma}\right\}$. From Lemma 2A, for all $v \in \mathcal{Y}$ such that $\theta(v)>w_{L}^{\sigma}, L$ strictly prefers implementing $p$ to $n$ given continuation play $\sigma$, and since $\sigma$ is an equilibrium, $v \notin \Upsilon^{\sigma}(p, n)$. Since $\sigma$ is an EE, $\mu\left(\Upsilon^{\sigma}(p, q)\right)=0$. By definition of $\Upsilon^{\sigma},\left(\Upsilon^{\sigma}(p, x)\right)_{x \in\{n, p, q\}}$ is a partition of $\Upsilon$, so necessarily, $v \in \Upsilon^{\sigma}(p, p)$. Conversely, for all $v \in \Upsilon$ such that $\theta(v)<w_{L}^{\sigma}$, from Step A1, $\theta(v)<w_{R}^{\sigma}$, so both players strictly prefer implementing $n$ to $p$ given continuation play $\sigma$. Since $\sigma$ is an equilibrium, necessarily, $v \notin \Upsilon^{\sigma}(p, p)$. Since $F$ is continuous, the set of $v$ such that $\theta(v)=w_{L}^{\sigma}$ has probability 0 , which completes the proof of the first equality in Step A3.

From Lemma 2A, for all $v \in \Upsilon$ such that $\theta(v)<w_{R}^{\sigma}, R$ strictly prefers implementing $n$ to $p$ given continuation play $\sigma$, and since $\sigma$ is an equilibrium, $v \notin \Upsilon^{\sigma}(n, p)$. Since $\sigma$ is an EE, $\mu\left(\Upsilon^{\sigma}(n, q)\right)=0$. Since $\left(\Upsilon^{\sigma}(n, x)\right)_{x \in\{n, p, q\}}$ is a partition of $\Upsilon$, necessarily, $v \in$ $\Upsilon^{\sigma}(n, n)$. Conversely, for all $v \in \Upsilon$ such that $\theta(v)>w_{R}^{\sigma}$, from Step A1, $\theta(v)>w_{L}^{\sigma}$, so from Lemma 2A, both players strictly prefer implementing $p$ to $n$, given continuation play $\sigma$. Therefore, $v \notin \Upsilon^{\sigma}(n, n)$. The second equality in Step A3 follows then from the continuity of $F$.

Step A4: $\left(w_{L}^{\sigma}, w_{R}^{\sigma}\right)$ satisfies (12) and $w_{L}^{\sigma}<w_{L}<w_{R}<w_{R}^{\sigma}$. From Step A3, modulo a zero measure set, $\Upsilon^{\sigma}(p, p) \cap \Upsilon^{\sigma}(n, n)=\left\{v \in \Upsilon: \theta(v) \in\left(w_{L}^{\sigma}, w_{R}^{\sigma}\right)\right\}$. Substituting this equality into (16), we obtain (12). Step Al and the assumption that $F$ has full support 
imply $\int_{w_{L}^{\sigma}}^{w_{R}^{\sigma}}\left(w_{L}^{\sigma}-\theta\right) d F(\theta)<0<\int_{w_{L}^{\sigma}}^{w_{R}^{\sigma}}\left(w_{R}^{\sigma}-\theta\right) d F(\theta)$. Together with (12), the above inequalities imply $w_{L}^{\sigma}<w_{L}<w_{R}<w_{R}^{\sigma}$.

Step A5: $e_{L}^{\sigma}>0$. Let us first prove that $\mu\left(\Upsilon^{\sigma}(p, n) \cap \Upsilon^{\sigma}(q, q)\right)=0$. Suppose to the contrary that the latter probability is positive. Then from Lemma 2A, for some $v \in Y^{\sigma}(p, n) \cap \Upsilon^{\sigma}(q, q)$, both players strictly prefer implementing $n$ to $p$ and one player weakly prefers implementing $q$ to $n$, given continuation play $\sigma$. From Lemma 2A, this means that $\min _{i}\left\{w_{i}^{\sigma}+e_{i}^{\sigma}\right\} \leq \theta(v)<w_{L}^{\sigma}$ and, therefore, $\min _{i}\left\{w_{i}^{\sigma}+e_{i}^{\sigma}\right\}<w_{L}^{\sigma}$, a contradiction to Steps A1 and A2.

Substituting $\mu\left(\Upsilon^{\sigma}(p, n) \cap \Upsilon^{\sigma}(q, q)\right)=0$ into (8), we get

$$
\frac{e_{i}^{\sigma}-e_{i}}{\delta}=\int_{\Upsilon^{\sigma}(p, p) \cap \Upsilon^{\sigma}(q, q)} e_{i}^{\sigma} d \mu(v)+\int_{\Upsilon^{\sigma}(p, p) \cap \Upsilon^{\sigma}(q, n)}\left(\theta(v)-w_{i}^{\sigma}\right) d \mu(v) .
$$

From Step A3, for almost all $v \in Y^{\sigma}(p, p), \theta(v)>w_{L}^{\sigma}$, so (17) implies $e_{L}^{\sigma} \geq e_{L}>0$.

Step A6: $\Upsilon^{\sigma}(q, q) \subseteq \Upsilon^{\sigma}(p, p)$. Let $v \in \Upsilon^{\sigma}(q, q)$. Since $\sigma$ is an equilibrium, in state $v$, one player weakly prefers implementing $q$ to $n$ given continuation play $\sigma$, so $\theta(v) \geq$ $\min \left\{w_{L}^{\sigma}+e_{L}^{\sigma}, w_{R}^{\sigma}+e_{R}^{\sigma}\right\}$. From Steps A1, A2, and A5, the latter inequality implies $\theta(v)>$ $w_{L}^{\sigma}$. From Step A3, $v \in Y^{\sigma}(p, p)$.

Part B. Recall that an EE is an equilibrium in which $q$ is never implemented on path given initial status quo $n$. This definition does not prevent players from proposing and implementing $q$ under status quo $q$, and this may happen in an EE $\sigma$ such that $e_{k}^{\sigma}=0$ for some $k$, for such EE, formula (12) is more complicated. So in this part, we prove that for any $\mathrm{EE} \sigma$, there always exists an EE $\sigma^{\prime}$ such that $w^{\sigma^{\prime}}=w^{\sigma}$ and $q$ is never implemented even under status quo $q$. We construct $\sigma^{\prime}$ as the limit of a sequence $\left(\sigma^{k}\right)_{k \in \mathbb{N}}$ defined recursively from $\sigma$ as follows. We set $\sigma^{0}=\sigma$. For any $k \geq 0$, we define $\sigma^{k+1}$ as a function of $\sigma^{k}$ as follows. Consider first the action nodes of veto player $i \in\{L, R\}$. When comparing $n$ and $p, i$ plays $\sigma$. When comparing $p$ and $q, i$ votes for $p$. When comparing $n$ and $q, i$ votes for $q$ when $\theta(v)>w_{i}^{\sigma}+e_{i}^{\sigma^{k}}$ and for $n$ otherwise. Consider now the action nodes of proposer $i \in\{L, R\}$. When the status quo is $n$ or $p, i$ plays $\sigma$. When the status quo is $q, L$ proposes $n$ when $\theta \leq w_{L}^{\sigma}$ and $p$ otherwise, and $R$ proposes $n$ when $\theta \leq \min \left\{w_{L}^{\sigma}+e_{L}^{\sigma^{k}}, w_{R}^{\sigma}\right\}$ and $p$ otherwise.

Step B1: For all $k \in \mathbb{N}, w^{\sigma^{k}}=w^{\sigma}$ and $e_{L}^{\sigma^{1}} \leq e_{L}^{\sigma^{0}}$. By construction, for all $k \in \mathbb{N}, \sigma^{k}$ prescribes the same actions as $\sigma$ when neither the status quo nor the proposal is $q$, and these actions never lead to outcome $q$. So the value of the game $\Gamma$ with initial status quo $n(p)$ is the same with continuation play $\sigma^{k}$ and $\sigma$. From Lemma 2A, this implies $w^{\sigma^{k}}=w^{\sigma}$. When the status quo is $q$, by construction of $\sigma^{1}, \sigma^{1}$ always implements $n$ or $p$, and $\sigma^{1}$ prescribes actions that are subgame perfect given continuation play $\sigma$. So the actions prescribed by $\sigma$ and $\sigma^{1}$ under status quo $q$ may differ only in an action node in which one player is indifferent between two actions. Lemma 2A implies that a given player can be indifferent between implementing $n$ and $p$ ( $n$ and $q$ ) only in a unique state. Since $F$ is continuous, this means that she can be indifferent between two actions in a nonnegligible set of states only when one action leads to outcome $p$ whereas the other leads to outcome $q$ (and when $e_{R}^{\sigma}=0$ ). In those cases, $\sigma^{1}$ always prescribes $R$ to choose $p$, which is what $L$ prefers given continuation play $\sigma$ since from part A of this lemma, 
$e_{L}^{\sigma}>0$. So under status quo $q$, $L$ is weakly better off when $\sigma^{1}$ is played than when $\sigma$ is played in the current period, given continuation play $\sigma$. Since the continuation paths of $\sigma^{1}$ and $\sigma$ coincide under status quo $n$ or $p$, this implies that under status quo $q$, $L$ weakly prefers continuation play $\sigma^{1}$ to continuation play $\sigma$. From Lemma 2A, this means that $e_{L}^{\sigma^{1}} \leq e_{L}^{\sigma^{0}}$.

Step B2: For all $k \in \mathbb{N}, e_{L}^{\sigma^{k}}>0$ and

$$
\frac{e_{i}^{\sigma^{k+1}}-e_{i}}{\delta}=\int_{w_{L}^{\sigma}}^{\min \left\{w_{L}^{\sigma}+e_{L}^{\sigma^{k}}, w_{R}^{\sigma}\right\}} b_{R}(\theta, q)\left(\theta-w_{i}^{\sigma}\right) d F(\theta) .
$$

We prove Step B2 by induction. Since $\sigma^{0}=\sigma$ is an EE, part A of this lemma implies $e_{L}^{\sigma^{0}}>0$. Suppose now $e_{L}^{\sigma^{k}}>0$ for some $k \in \mathbb{N}$, and let us show (18) and $e_{L}^{\sigma^{k+1}}>0$. From Lemma $2 \mathrm{~A},\left(e_{L}^{\sigma^{k+1}}-e_{i}\right) / \delta$ is the expected payoff gain from having status quo $p$ instead of $q$ given continuation play $\sigma^{k+1}$. To compute it, note that from part A of this lemma, $w_{L}^{\sigma}<w_{R}^{\sigma}$, and since $e_{L}^{\sigma^{k}}>0$, we have $w_{L}^{\sigma}<\min \left\{w_{L}^{\sigma}+e_{L}^{\sigma^{k}}, w_{R}^{\sigma}\right\}$. So by construction of $\sigma^{k+1}$, on path, status quo $q$ and $p$ both lead to outcome $n$ when $\theta<w_{L}^{\sigma}$, and they both lead to outcome $p$ when $\theta>w_{R}^{\sigma}$, when $\theta \in\left(w_{L}^{\sigma}, w_{R}^{\sigma}\right)$ and $L$ is the proposer, and when $\theta \in\left(\min \left\{w_{L}^{\sigma}+e_{L}^{\sigma^{k}}, w_{R}^{\sigma}\right\}, w_{R}^{\sigma}\right)$ and $R$ is the proposer. Modulo a negligible set of states, the only remaining case is when $R$ is the proposer and $\theta \in\left(w_{L}^{\sigma}, \min \left\{w_{L}^{\sigma}+e_{L}^{\sigma^{k}}, w_{R}^{\sigma}\right\}\right)$. In such cases, status quo $q$ and $p$ lead to outcome $n$ and $p$, respectively, so from Lemma 2A, the relative continuation payoff gain is $\theta-w_{i}^{\sigma^{k+1}}=\theta-w_{i}^{\sigma}$. Integrating over all these cases, we obtain (18). From part A of this lemma, $w_{L}^{\sigma}<w_{R}^{\sigma}$, so (18) implies $e_{L}^{\sigma^{k+1}}>e_{L}>0$.

Step B3: For all $k \geq 1, e_{L}^{\sigma^{k+1}} \leq e_{L}^{\sigma^{k}}$ and $e_{R}^{\sigma^{k+1}} \geq e_{R}^{\sigma^{k}}$. We prove Step B3 by induction. From Steps B1 and B2, $e_{L}^{\sigma^{1}} \leq e_{L}^{\sigma^{0}}$. Suppose now $e_{L}^{\sigma^{k+1}} \leq e_{L}^{\sigma^{k}}$ for some $k \in \mathbb{N}$. From Step B2,

$$
e_{i}^{\sigma^{k+2}}-e_{i}^{\sigma^{k+1}}=\delta \int_{\min \left\{w_{L}^{\sigma}+e_{L}^{\sigma^{k}}, w_{R}^{\sigma}\right\}}^{\min \left\{w_{R}^{\sigma}+e^{\sigma^{k+1}}, w_{R}^{\sigma}\right\}} b_{R}(\theta, q)\left(\theta-w_{i}^{\sigma}\right) d F(\theta) .
$$

Since $e_{L}^{\sigma^{k}} \leq e_{L}^{\sigma^{k-1}}$, the right-hand side of the above equality is negative for $i=L$ and positive for $i=R$.

Step B4: $\sigma^{k+1}$ prescribes subgame-perfect actions given continuation play $\sigma^{k}$. From Part A of this lemma, $e_{R}^{\sigma^{0}} \geq 0$. Steps B2 and B3 imply then that for all $i \in\{L, R\}$ and $k \in \mathbb{N}$, $e_{i}^{\sigma^{k}} \geq 0$. So using Lemma $2 \mathrm{~A}$, one can check check that by construction of $\left(\sigma^{k}\right)_{k \in \mathbb{N}}, \sigma^{k+1}$ prescribes the veto player to approve (reject) the proposal when she strictly prefers the proposal (status quo) given continuation play $\sigma^{k}$, and that $\sigma^{k+1}$ prescribes the proposer to propose the policy that she prefers among those accepted by the veto player.

Step B5: $\sigma^{\prime} \equiv \lim _{k \rightarrow \infty} \sigma$ exists and satisfies the properties stated in part B. From Steps $\mathrm{B} 1$ and B3, $w_{i}^{\sigma^{k}}$ and $e_{i}^{\sigma^{k}}$ have a limit as $k \rightarrow \infty$, and since $\sigma^{k+1}$ is a cutoff strategy in which the cutoffs are continuous functions of $w_{i}^{\sigma^{k}}$ and $e_{i}^{\sigma^{k}}$, it has a limit $\sigma^{\prime}$. From Step B4, by continuity, $\sigma^{\prime}$ is an equilibrium, and by construction of $\left(\sigma^{k}\right)_{k \in \mathbb{N}}, \sigma^{\prime}$ never prescribes proposal $q$, so $\sigma$ is an EE. Taking the limit in (18), we obtain (13). 
Part C. Suppose $\left(w^{*}, e^{*}\right) \in \mathbb{R}^{4}$ solves (14) and (15) with $e_{R}^{*} \geq 0$. Construct a strategy profile $\sigma$ as follows. Consider first the action nodes of veto player $i \in\{L, R\}$. When comparing $n$ and $p, i$ votes for $p$ when $\theta>w_{i}^{*}$ and for $n$ otherwise. When comparing $p$ and $q, i$ votes for $p$. When comparing $n$ and $q, i$ votes for $q$ when $\theta(v)>w_{i}^{*}+e_{i}^{*}$ and for $n$ otherwise. Consider now the action nodes of proposer $i \in\{L, R\}$. When the status quo is $n$ or $p, i$ proposes $p$ when $\theta>w_{i}^{*}$ and $n$ otherwise, When the status quo is $q, L$ proposes $n$ when $\theta \leq w_{L}^{*}$ and $p$ otherwise, and $R$ proposes $n$ when $\theta \leq \min \left\{w_{L}^{*}+e_{L}^{*}, w_{R}^{*}\right\}$ and $p$ otherwise. By construction, $q$ is never implemented on path. Therefore, to complete the proof, it suffices to show that $\sigma$ is an equilibrium. From Lemma 2B, it suffices to show that $\sigma$ is subgame perfect given continuation payoff parameters $\left(w^{*}, e^{*}\right)$, and that $\left(w^{\sigma}, e^{\sigma}\right)$ (as defined in Lemma 2A) coincides with $\left(w^{*}, e^{*}\right)$. This is what Steps C1 to C3 below do.

Step C1: $\sigma$ is subgame perfect for the continuation payoff parameters $\left(w^{*}, e^{*}\right)$ in the sense of Definition 2. By assumption, $e_{R}^{*} \geq 0$, and since $e^{*}$ satisfies (15), we have $e_{L}^{*} \geq e_{L}>0$, so both players always weakly prefer to implement $p$ to $q$. So one can easily check that by construction of $\sigma, \sigma$ prescribes either veto players to accept (reject) a proposal when it gives her a greater (lesser) continuation payoff, given continuation payoff parameters $\left(w^{*}, e^{*}\right)$. Likewise, $\sigma$ prescribes either proposer to propose the policy that gives her the greatest continuation payoff among those accepted by the veto player, given continuation payoff parameters $\left(w^{*}, e^{*}\right)$.

Step C2: $w^{\sigma}=w^{*}$. Since $w^{*}$ satisfies (14), the same reasoning as in Step A1 implies $w_{L}^{*}<w_{R}^{*}$. By construction of $\sigma, q$ is never implemented on path, and status quo $n$ and $p$ lead to different outcomes only when $\theta \in\left(w_{L}^{*}, w_{R}^{*}\right)$, in which case either status quo stay in place, and the corresponding continuation payoff gain is $w_{i}^{\sigma}-\theta$. From Lemma 2A, this means that $\frac{w_{i}^{\sigma}-w_{i}}{\delta}=\int_{w_{L}^{*}}^{w_{*}^{*}}\left(w_{i}^{\sigma}-\theta\right) d F(\theta)$. This equation can be viewed as a linear equation in $w_{i}^{\sigma}$, and it has a unique solution. From (14), $w_{i}^{*}$ is also a solution to that equation, so $w_{i}^{\sigma}=w_{i}^{*}$.

Step C3: $e^{\sigma}=e^{*}$. The same reasoning as in Step B2 (taking $w^{\sigma}=w^{*}$ and $e_{L}^{\sigma^{k}}=e_{L}^{*}$ ) shows that by construction of $\sigma$, modulo a negligible set of states, status quo $p$ and $q$ lead to different outcomes only when $R$ is the proposer and $\theta \in\left(w_{L}^{*}, \min \left\{w_{L}^{*}+e_{L}^{*}, w_{R}^{*}\right\}\right)$, in which case status quos $p$ and $q$ lead to outcomes $p$ and $n$, respectively, and the corresponding continuation payoff gain is $\theta-w_{i}^{*}$. Therefore, Lemma $2 \mathrm{~A}$ implies that $\frac{e_{i}^{\sigma}-e_{i}}{\delta}=\int_{w_{L}^{*}}^{\min \left\{w_{L}^{*}+e_{L}^{*}, w_{R}^{*}\right\}} b_{R}(\theta, q)\left(\theta-w_{i}^{*}\right) d F(\theta)$. Together with (15), that equation implies $e_{i}^{\sigma}=e_{i}^{*}$.

\section{A.2 Proofs for Section 4}

Proof of Lemma 1. The second claim of Lemma 1 follows from Lemma 2A. The rest of the proof establishes the first claim, i.e., equilibrium existence. For all $i \in\{L, R\}$, let $\Pi_{i}$ 
be a correspondence defined on $\mathbb{R}$ such that for all $e_{i}^{c} \in \mathbb{R}$,

$$
\Pi_{i}\left(e_{i}^{c}\right) \equiv \begin{cases}{[0,1]} & \text { if } e_{i}^{c}=0 \\ 0 & \text { if } e_{i}^{c}<0 \\ 1 & \text { if } e_{i}^{c}>0\end{cases}
$$

Define the correspondence $\Pi \equiv\left(\Pi_{L}, \Pi_{R}\right)$.

The structure of the proof is as follows. In Step 1, for any continuation payoff parameters $\left(w^{c}, e^{c}\right) \in \mathbb{R}^{4}$, we construct a set of strategy profiles that are subgame perfect for the continuation payoff parameters $\left(w^{c}, e^{c}\right)$ in the sense of Definition 2. In particular, for any given $\left(w^{c}, e^{c}\right)$, player $i$ has strict preferences between $n$ and $p$ and between $n$ and $q$ for almost all realizations of $\theta$, and we assume then that when indifferent, player $i$ takes action that results in the implementation of $n$. Hence, we uniquely determine the subgame-perfect behavior of player $i$ when choosing between $n$ and any intervention. When choosing between $p$ and $q$, we allow each player to randomize, and we use $\pi_{i}$ to denote the probability that $i$ chooses to implement $q$. Hence, for any $\left(w^{c}, e^{c}\right)$ and any mixing rule $\pi \in[0,1]^{2}$, we construct a unique strategy profile. Let $\sigma\left(\pi, w^{c}, e^{c}\right)$ denote this strategy profile, and let $w^{\sigma\left(\pi, w^{c}, e^{c}\right)}$ and $e^{\sigma\left(\pi, w^{c}, e^{c}\right)}$ be the unique continuation payoff parameters associated with this strategy profile as defined in Lemma 2. From (19), whenever $\pi \in \Pi\left(e^{c}\right), \sigma\left(\pi, w^{c}, e^{c}\right)$ is subgame-perfect for the continuation payoff parameters $\left(w^{c}, e^{c}\right)$. We define the correspondence $\Sigma$ that maps $\mathbb{R}^{4} \times[0,1]^{2}$ into itself $\mathrm{as}^{24}$

$$
\Sigma\left(w^{c}, e^{c}, \pi\right) \equiv\left\{\left(w^{\sigma\left(\pi, w^{c}, e^{c}\right)}, e^{\sigma\left(\pi, w^{c}, e^{c}\right)}, \pi^{\prime}\right): \pi^{\prime} \in \Pi\left(e^{\sigma\left(\pi, w^{c}, e^{c}\right)}\right)\right\} .
$$

Note that the strategies used to define $\Sigma$ are subgame perfect only for $\pi \in \Pi\left(e^{c}\right)$ and not for all $\pi \in[0,1]^{2}$, but by construction of $\Sigma$, if $\left(\pi^{*}, w^{*}, e^{*}\right)$ is a fixed point of $\Sigma$, $\pi^{*} \in \Pi\left(e^{*}\right)$ and $\sigma\left(\pi^{*}, w^{*}, e^{*}\right)$ is subgame perfect for the continuation payoff parameters $\left(w^{*}, e^{*}\right)=\left(w^{\sigma\left(\pi^{*}, w^{*}, e^{*}\right)}, e^{\sigma\left(\pi^{*}, w^{*}, e^{*}\right)}\right)$. From Lemma $2 \mathrm{~B}$, this means that $\sigma\left(\pi^{*}, w^{*}, e^{*}\right)$ is an equilibrium. Thus, to prove equilibrium existence, it suffices to show that $\Sigma$ has a fixed point. The rest of the proof then checks that $\Sigma$ satisfies the conditions of Kakutani's fixed point theorem. Step 2 shows that there exists a compact and convex set $C$ such that $\Sigma(C) \subset C$, Steps 3 and 4 show that the graph of $\Sigma$ is closed, and Step 5 shows that $\Sigma$ is convex-valued.

Step 1. For each $\left(\pi, w^{c}, e^{c}\right)$, we construct a strategy profile $\sigma\left(\pi, w^{c}, e^{c}\right)$ that is subgame perfect for the continuation payoff parameters $\left(w^{c}, e^{c}\right)$ (in the sense of Definition 2) whenever players choose between $n$ and $p$ or $n$ and $q$, but uses $\pi$ as the tie-breaking rule between $p$ and $q$. Whenever $\pi \in \Pi\left(e^{c}\right)$, this strategy profile is subgame perfect. The construction of $\sigma\left(\pi, w^{c}, e^{c}\right)$ is intuitive, but the detailed description below will be useful in

\footnotetext{
${ }^{24}$ Note that $\Sigma$ is not a mapping from strategy space to strategy space as is usual in fixed-point-type existence proofs. The reason for this is that the set of strategies that are subgame perfect for the continuation parameters $\left(w^{c}, e^{c}\right)$ may be quite complex whenever $e^{c}=0$, and showing convexity or contractibility of such a set may be an issue. Instead, we take as a primitive $\left(w^{c}, e^{c}\right)$ together with a tie-breaking rule, and map it into the payoff parameters generated by a strategy that uses this tie-breaking rule and is subgame perfect with respect to $\left(w^{c}, e^{c}\right)$ and map it into a set of new tie-breaking rule that are subgame perfect with respect to $e^{\sigma\left(\pi, w^{c}, e^{c}\right)}$.
} 
Step 3 to show that the continuation payoff parameters derived from $\sigma\left(\pi, w^{c}, e^{c}\right)$ are continuous in $\left(\pi, w^{c}, e^{c}\right)$. In this description, for brevity, players' preferences should be understood as their preferences given continuation payoff parameters $\left(w^{c}, e^{c}\right)$.

Veto player's strategy. In any Markov state in which the veto player $i \in\{L, R\}$ has the choice between $n$ and $p$ ( $n$ and $q$ ), $\sigma_{i}$ prescribes $i$ to choose $n$ when she weakly prefers implementing $n$, i.e., when $\theta \leq w_{i}^{c}$ (when $\theta \leq w_{i}^{c}+e_{i}^{c}$ ) and $\sigma_{i}$ prescribes $i$ to choose $p(q)$ otherwise.

When the veto player has to choose between $p$ and $q, \sigma_{i}$ prescribes $i$ to choose $p$ with probability (henceforth w.p.) $\pi_{i}$ and $q$ w.p. $1-\pi_{i}$, irrespective of $\theta$. From (19), when $\pi \in \Pi\left(e^{c}\right)$, this behavior is subgame perfect given continuation payoff parameters $\left(w^{c}, e^{c}\right)$.

Finally, when the status quo is proposed, the action prescribed by $\sigma_{i}$ is irrelevant.

Proposer's strategy under status quo $p$. When $i$ weakly prefers $p$ to $n$ or when $\sigma_{j}$ prescribes $j$ to veto proposal $n$ (i.e., when $\theta \geq w_{i}^{c}$ or $\theta>w_{j}^{c}$ ), then $\sigma_{i}$ prescribes proposer $i$ to propose $p$ w.p. $\pi_{i}$ and $q$ w.p. $1-\pi_{i}$ (note that by the preceding argument, the veto player strategy is independent of the proposer's strategy). When $\pi \in \Pi\left(e^{c}\right)$, this is subgame perfect given the veto player's strategy $\sigma_{j}$ and continuation payoff parameters $\left(w^{c}, e^{c}\right)$, because in both cases, $i$ always weakly prefers proposing the status quo $p$ to proposing $n$, and from (19), when $\pi \in \Pi\left(e^{c}\right), \sigma_{i}$ prescribes $i$ to propose $q$ only when she weakly prefers it to $p$.

The remaining case is when $i$ strictly prefers $n$ to $p$ and $\sigma_{j}$ prescribes $j$ to accept proposal $n$ (i.e., when $\theta_{i}<w^{c}$ and $\theta_{j} \leq w^{c}$ ). In this case, from Lemma 2A, the continuation payoff gain for $i$ from proposing $n$ instead of $p$ is $w_{i}^{c}-\theta$, whereas that of proposing $q$ instead of $p$ is $-\pi_{j} e_{i}^{c}$. So when $w_{i}^{c}-\theta>-\pi_{j} e_{i}^{c}$, proposal $n$ dominates both proposals $q$ and $p$, and we set $\sigma_{i}$ to prescribe $i$ to propose $n$. When $w_{i}^{c}-\theta \leq-\pi_{j} e_{i}^{c}$, proposal $n$ is dominated by proposal $q$. So $\sigma_{i}$ prescribes $i$ to propose $p$ w.p. $\pi_{i}$ and $q$ w.p. $1-\pi_{i}$. From (19), when $\pi \in \Pi\left(e^{c}\right)$, this is subgame perfect under status quo $p$ given the veto player's strategy $\sigma_{j}$ and continuation payoff parameters $\left(w^{c}, e^{c}\right)$.

Proposer's strategy under status quo q. The strategy is analogous to the proposer's strategy under status quo $p$, where the role of $p$ and $q$ and their corresponding continuation payoff parameters are reversed.

Proposer's strategy under status quo $n$. When $i$ weakly prefers $n$ to $p$ and $n$ to $q$ (i.e., when $\left.\theta \leq \min \left\{w_{i}^{c}, w_{i}^{c}+e_{i}^{c}\right\}\right), \sigma_{i}$ prescribes $i$ to propose $n$, which is optimal since $n$ is the status quo.

When $i$ strictly prefers $p$ to $n$ and weakly prefers $n$ to $q$ (i.e., when $w_{i}^{c}<\theta \leq w_{i}^{c}+e_{i}^{c}$ ), $\sigma$ prescribes $i$ to propose $p$, which is optimal for $i$ irrespective of whether $j$ accepts it.

When $i$ strictly prefers $q$ to $n$ and weakly prefers $n$ to $p$ (i.e., when $w_{i}^{c}+e_{i}^{c}<\theta \leq w_{i}^{c}$ ), $\sigma$ prescribes $i$ to propose $q$, which is optimal for $i$ irrespective of whether $j$ accepts it.

The only remaining case is when $i$ strictly prefers $q$ and $p$ to $n$ (i.e., when $\theta>$ $\left.\max _{i \in\{L, R\}}\left\{w_{i}^{c}, w_{i}^{c}+e_{i}^{c}\right\}\right)$. In that case, if $\sigma_{j}$ prescribes $j$ to veto both $p$ and $q$ (i.e., $\left.\theta \leq \min \left\{w_{j}^{c}, w_{j}^{c}+e_{j}^{c}\right\}\right), \sigma_{i}$ prescribes $i$ to propose $n$. If $\sigma_{j}$ prescribes $j$ to veto $q$ but not $p$ (i.e., $w_{j}^{c}<\theta \leq w_{j}^{c}+e_{j}^{c}$ ), $\sigma_{i}$ prescribes $i$ to propose $p$. If $\sigma_{j}$ prescribes $j$ to veto $p$ but not $q$ (i.e., $\left.w_{j}^{c}+e_{j}^{c}<\theta \leq w_{j}^{c}\right), \sigma_{i}$ prescribes $i$ to propose $q$. Finally, if both $p$ and $q$ are accepted (i.e., $\left.\theta>\min \left\{w_{j}^{c}, w_{j}^{c}+e_{j}^{c}\right\}\right), \sigma_{i}$ prescribes $i$ to propose $p$ w.p. $\pi_{i}$ and $q$ w.p. $1-\pi_{i}$. 
Step 2: There exists a compact and convex set $C$ such that $\Sigma(C) \subset C$. Let $B \equiv$ $\frac{\max _{i}\left\{\left|w_{i}\right|+\left|e_{i}\right|+E(|\theta|)\right\}}{1-\delta}$ and $C \equiv[-B, B]^{4} \times[0,1]^{2}$. The compactness and convexity of $C$ is obvious. To see why $\Sigma(C) \subseteq C$, note that from (2), the flow-payoff difference between any two policies is bounded by $E\left[|\theta|+\left|w_{i}\right|+\left|e_{i}\right|\right]$, so the expected discounted payoff difference between any two policy paths is bounded by $B \equiv \frac{E[|\theta|]+\left|w_{i}\right|+\left|e_{i}\right|}{1-\delta}$. Therefore, by definition of $w_{i}^{\sigma}$ and $e_{i}^{\sigma}$ (see Lemma 2A), for any strategy profile $\sigma,\left|w_{i}^{\sigma}\right|+\left|e_{i}^{\sigma}\right|$ is bounded by $B$.

Step 3: The functions $\left(\pi, w^{c}, e^{c}\right) \rightarrow w^{\sigma\left(\pi, w^{c}, e^{c}\right)}$ and $\left(\pi, w^{c}, e^{c}\right) \rightarrow e^{\sigma\left(\pi, w^{c}, e^{c}\right)}$ are continuous. By construction of $\sigma$ (see Step 1), for all $\theta \in \mathbb{R}, x, y \in\{n, p, q\},\left(\pi, w^{c}, e^{c}\right) \in C$, the probability that in state $\theta, \sigma\left(\pi, w^{c}, e^{c}\right)$ prescribes the proposer to propose $y$ (the veto player to accept $y$ ) under status quo $x$ is piecewise constant in $\theta$. Moreover, on each of the intervals on which it is constant in $\theta$, this probability is continuous in $\left(\pi, w^{c}, e^{c}\right)$, and the bounds of these intervals depend continuously on $\left(\pi, w^{c}, e^{c}\right)$. Therefore, the probability that on the path of $\sigma\left(\pi, w^{c}, e^{c}\right)$, status quo $x$ is replaced by policy $y$ in some state $\theta$ is continuous in $\left(\pi, w^{c}, e^{c}\right)$ except at a finite number of $\theta$. Using Notation 1 , the continuity of $F$ implies then that the expectation of $V_{i}^{\sigma\left(\pi, w^{c}, e^{c}\right)}(\theta, x)$ conditional on $\theta \sim F$ is continuous in $\left(\pi, w^{c}, e^{c}\right)$. From (9) and (10), this implies that $w_{i}^{\sigma\left(\pi, w^{c}, e^{c}\right)}$ and $e_{i}^{\sigma\left(\pi, w^{c}, e^{c}\right)}$ are continuous in $\left(\pi, w^{c}, e^{c}\right)$.

Step 4: $\Sigma$ has a closed graph. Let $\left(\pi^{k}, w^{k}, e^{k}\right)_{k \in \mathbb{N}}$ and $\left(\hat{\pi}^{k}, \hat{w}^{k}, \hat{e}^{k}\right)_{k \in \mathbb{N}}$ be two sequences in $C$ that converge to some $\left(\pi^{\infty}, w^{\infty}, e^{\infty}\right)$ and $\left(\hat{\pi}^{\infty}, \hat{w}^{\infty}, \hat{e}^{\infty}\right)_{k \in \mathbb{N}}$, respectively, and such that for all $k \in \mathbb{N},\left(\hat{\pi}^{k}, \hat{w}^{k}, \hat{e}^{k}\right) \in \Sigma\left(\pi^{k}, w^{k}, e^{k}\right)$. We need to show that $\left(\hat{\pi}^{\infty}, \hat{w}^{\infty}, \hat{e}^{\infty}\right) \in \Sigma\left(\pi^{\infty}, w^{\infty}, e^{\infty}\right)$. From (20) and (19), $\left(\hat{\pi}^{k}, \hat{w}^{k}, \hat{e}^{k}\right) \in \Sigma\left(\pi^{k}, w^{k}, e^{k}\right)$ implies that $\hat{w}^{k}=w^{\sigma\left(\pi^{k}, w^{k}, e^{k}\right)}, \hat{e}^{k}=e^{\sigma\left(\pi^{k}, w^{k}, e^{k}\right)}$, and $\hat{\pi}^{k} \in \Pi\left(\hat{e}^{k}\right)$. From Step 3, $\hat{w}^{\infty}=$ $w^{\sigma\left(\pi^{\infty}, w^{\infty}, e^{\infty}\right)}$ and $\hat{e}^{\infty}=e^{\sigma\left(\pi^{\infty}, w^{\infty}, e^{\infty}\right)}$. So it remains to establish that $\hat{\pi}^{\infty} \in \Pi\left(\hat{e}^{\infty}\right)$, which requires that $\hat{e}^{\infty}>0$ implies $\hat{\pi}^{\infty}=1$ and $\hat{e}^{\infty}<0$ implies $\hat{\pi}^{\infty}=0$. Note that $\hat{e}^{\infty}>0 \mathrm{im}$ plies $\hat{e}^{k}>0$ and, thus, $\hat{\pi}^{k}=1$ for $k$ large enough, so $\hat{e}^{\infty}=1$. A similar argument establishes the second implication.

Step 5: $\Sigma$ is convex-valued. One can immediately check from (19) that $\Pi(\cdot)$ is convexvalued, so from (20), $\Sigma$ is also convex-valued.

Proof of Proposition 2. Let $\sigma$ be an arbitrary IE and let $i, j \in\{L, R\}$ be such that $w_{i}^{\sigma} \leq$ $w_{j}^{\sigma}$ in this equilibrium.

Step 1: $\mu\left(\Upsilon^{\sigma}(p, p) \cap \Upsilon^{\sigma}(q, n)\right)>0$. Since $\sigma$ is an equilibrium, in any state $v \in$ $\Upsilon^{\sigma}(p, n)$, each player $k$ must prefer implementing $n$ to $p$ given continuation play $\sigma$, so Lemma $2 \mathrm{~A}$ implies $\theta(v) \leq w_{k}^{\sigma}$ and, therefore, $w_{k}^{\sigma}+e_{k}^{\sigma}-\theta(v) \geq e_{k}^{\sigma}$. Substituting the latter inequality in the equilibrium formula for $e_{k}^{\sigma}((8))$, we obtain

$$
\begin{aligned}
& \frac{e_{k}^{\sigma}-e_{k}}{\delta} \geq \int_{\left(\Upsilon^{\sigma}(p, p) \cup \Upsilon^{\sigma}(p, n)\right) \cap \Upsilon^{\sigma}(q, q)} e_{k}^{\sigma} d \mu(v)+\int_{\Upsilon^{\sigma}(p, p) \cap \Upsilon^{\sigma}(q, n)}\left(\theta(v)-w_{k}^{\sigma}\right) d \mu(v) \\
& \Rightarrow \quad e_{k}^{\sigma} \geq \frac{e_{k}+\delta \int_{\Upsilon^{\sigma}(p, p) \cap \Upsilon^{\sigma}(q, n)}\left(\theta(v)-w_{k}^{\sigma}\right) d \mu(v)}{1-\delta \mu\left(\left(\Upsilon^{\sigma}(p, p) \cup \Upsilon^{\sigma}(p, n)\right) \cap \Upsilon^{\sigma}(q, q)\right)} .
\end{aligned}
$$

Suppose to the contrary that $\mu\left(\Upsilon^{\sigma}(p, p) \cap \Upsilon^{\sigma}(q, n)\right)=0$. Then (21) implies $e_{L}^{\sigma}>0$ and $e_{R}^{\sigma}>0$, so both players always strictly prefer to implement $p$ to $q$ given continuation 
play $\sigma$. Since $\sigma$ is an equilibrium, this implies that $q$ is never implemented on path, which is impossible since $\sigma$ is an IE.

Step 2: $\min _{k \in\{L, R\}} w_{k}^{\sigma}<\min _{k \in\{L, R\}}\left(w_{k}^{\sigma}+e_{k}^{\sigma}\right)$. Since $\sigma$ is an equilibrium, for all $v \in$ $\Upsilon^{\sigma}(q, n)$, both players weakly prefer implementing $n$ to $q$ given continuation play $\sigma$, so from Lemma 2A, $\theta(v) \leq w_{k}^{\sigma}+e_{k}^{\sigma}$ for both $k \in\{L, R\}$. For all $v \in \Upsilon^{\sigma}(p, p)$, at least one player weakly prefers implementing $p$ to $n$, i.e., $\theta(v) \geq w_{k}^{\sigma}$ for some $k \in\{L, R\}$. So for all $v \in \mu\left(\Upsilon^{\sigma}(p, p) \cap \Upsilon^{\sigma}(q, n)\right), \min _{k \in\{L, R\}} w_{k}^{\sigma} \leq \theta(v) \leq \min _{k \in\{L, R\}}\left(w_{k}^{\sigma}+e_{k}^{\sigma}\right)$. Since from Step $1, \Upsilon^{\sigma}(p, p) \cap \Upsilon^{\sigma}(q, n)$ has positive probability and since $F$ is continuous, the latter weak inequalities must be strict for some $v \in \Upsilon^{\sigma}(p, p) \cap \Upsilon^{\sigma}(q, n)$, which implies Step 2.

Step 3: $e_{j}^{\sigma} \leq 0$ and $e_{i}^{\sigma}>e_{i}>0$. Since $\sigma$ is an equilibrium, for all $v \in \Upsilon^{\sigma}(p, p)$, some player weakly prefers implementing $p$ to $n$, so $\theta(v) \geq \min _{k \in\{L, R\}} w_{k}^{\sigma}=w_{i}^{\sigma}$. Since $F$ is continuous, this inequality is strict for almost all $v \in \Upsilon^{\sigma}(p, p)$. Substituting $\theta(v)>w_{i}^{\sigma}$ and Step 1 into (21), we get $e_{i}^{\sigma}>e_{i}>0$. As argued in Step 1, since $\sigma$ is an IE, $e_{i}^{\sigma}>0$ implies $e_{j}^{\sigma} \leq 0$.

Step 4: $w_{i}^{\sigma}<w_{j}^{\sigma}$. From Step 3, $e_{j}^{\sigma} \leq 0$, so (21) implies $\int_{v \in \Upsilon^{\sigma}(p, p) \cap \Upsilon^{\sigma}(q, n)}(\theta(v)-$ $\left.w_{j}^{\sigma}\right) d \mu(v)<0$. Therefore, there exists $\Upsilon^{o} \subset \Upsilon^{\sigma}(p, p) \cap \Upsilon^{\sigma}(q, n)$ such that $\mu\left(\Upsilon^{o}\right)>0$ and for all $v^{o} \in Y^{o}, \theta\left(v^{o}\right)<w_{j}^{\sigma}$. From Lemma 2A, the latter inequality implies that for all $v^{o} \in \Upsilon^{o}, j$ strictly prefers implementing $n$ to $p$ given continuation play $\sigma$. Since $\Upsilon^{o} \subset \Upsilon^{\sigma}(p, p)$ and $\sigma$ is an equilibrium, $i$ must weakly prefer implementing $p$ to $n$ in such states $v^{o}$, i.e., $\theta\left(v^{o}\right) \geq w_{i}^{\sigma}$. The latter inequality, together with $\theta\left(v^{o}\right)<w_{j}^{\sigma}$, implies $w_{i}^{\sigma}<w_{j}^{\sigma}$.

Proof of Proposition 3(i) And (ii) and part (iii) In the Case $w_{R}-w_{L} \rightarrow 0$.

An equilibrium is either an EE or an IE, so all equilibria are IE if and only if no EE exist. Lemma 3 implies that no EE exist if and only if there exists no $\left(w^{*}, e^{*}\right) \in \mathbb{R}^{2}$ such that $e_{R}^{*} \geq 0$, and (14) and (15) hold. The proof proceeds by deriving conditions on the primitive parameters such that any solution $\left(w^{*}, e^{*}\right)$ to (14) and (15) must be such that $e_{R}^{*}<0$.

Step 0: Notation. Condition (14) depends on $(w, e)$ only through $w$, so let $W^{*}(w)$ denote the set of $w^{*} \in \mathbb{R}^{2}$ solving (14) for $i=L, R$. If we fix $w^{*}$ and $e_{L}$, condition (15) for $i=L$ can be viewed as a fixed point in $e_{L}^{*}$; it depends on $(w, e)$ only through $w^{*}$ and $e_{L}$, so let $E_{L}^{*}\left(w^{*}, e_{L}\right)$ denote the set of $e_{L}^{*}$ solving (15) for $i=L$. Since the right-hand side of (15) is continuous and bounded in $e_{L}^{*}, E_{L}^{*}\left(w^{*}, e_{L}\right)$ is nonempty and closed, so let $e_{L}^{*}\left(w^{*}, e_{L}\right)$ denote its minimum. Finally, if we fix $w^{*}, e_{L}^{*}$, and $e_{R}$, (15) for $i=R$ pins down a unique $e_{R}^{*}$, which we denote $e_{R}^{*}\left(w^{*}, e_{L}^{*}, e_{R}\right)$.

Step 1: For all $w^{*} \in W^{*}(w), e_{L}^{*}\left(w^{*}, e_{L}\right)$ is weakly increasing in $e_{L}$, and $e_{R}^{*}\left(w^{*}, e_{L}^{*}, e_{R}\right)$ is weakly increasing in $e_{R}$ and weakly decreasing in $e_{L}^{*}$. Since the right-hand side of (15) for $i=L$ is continuous in $\left(e_{L}, e_{L}^{*}\right)$ and weakly increasing in $e_{L}$, Theorem 1 in Villas-Boas (1997) implies that the smallest fixed point $e_{L}^{*}\left(w^{*}, e_{L}\right)$ is weakly increasing in $e_{L}$. The comparative statics on $e_{R}^{*}$ (.) follow readily from (15) for $i=R$ and the fact that for all $w^{*} \in W^{*}(w), w_{L}^{*}<w_{R}^{*}$ (see Lemma 3A). 
Step 2: No EE exists if and only if for all $w^{*} \in W(w), e_{R}^{*}\left(w^{*}, e_{L}^{*}\left(w^{*}, e_{L}\right), e_{R}\right)<0$. As explained at the beginning of this proof, no EE exists if and only if for all solutions to (14) and (15), $e_{R}^{*}<0$. Using the notations of Step 0 and the comparative statics established in Step 1, no EE exists if and only if $e_{R}^{*}\left(w^{*}, e_{L}^{*}\left(w^{*}, e_{L}\right), e_{R}\right)<0$ for all $w^{*} \in W^{*}(w)$.

Step 3: Proof of Proposition 3(i) and (ii). Part (ii) follows from Step 2 together with the comparative statics on $e_{L}$ established in Step 1. Likewise, Step 2 together with the comparative statics on $e_{R}$ established in Step 1 proves part (i) for some $\bar{e}_{R} \in[0,+\infty]$. To complete the proof of part (ii), it suffices to show that $0<\bar{e}_{R}<$ $+\infty$.

To show that $\bar{e}_{R}<+\infty$, observe first that for any $w^{*}$ and $e_{L}^{*}$, the right-hand side of (15) for $i=R$ is bounded below by $-E[|\theta|]-w_{i}^{*}$, which is independent of $e_{L}^{*}$ and $e_{R}$. So for any $w^{*}$, for $e_{R}$ sufficiently large, $e_{R}^{*}\left(w^{*}, e_{L}^{*}\left(w^{*}, e_{L}\right), e_{R}\right)>0$, so an EE exists, as needed.

Let us now show that $\bar{e}_{R}>0$. Let $w^{*} \in W^{*}(w)$. As shown in Step A1 in the proof of Lemma 3, $w_{L}^{*}<w_{R}^{*}$, so (15) for $i=L$ implies that $e_{L}^{*}\left(w^{*}, e_{L}\right) \geq e_{L}>0$. Finally, (15) for $i=R$ implies

$$
\lim _{e_{R} \rightarrow 0} e_{R}^{*}\left(w^{*}, \underline{e_{L}^{*}}\left(w^{*}, e_{L}\right), e_{R}\right)=\delta \int_{w_{L}^{*}}^{\min \left\{w_{L}^{*}+\underline{e}_{L}^{*}\left(w^{*}, e_{L}\right), w_{R}^{*}\right\}} b_{R}(\theta, q)\left(\theta-w_{R}^{*}\right) d F(\theta) .
$$

Since $F$ has full support and $b_{R}(\theta, q)>\underline{b}>0$ for all $\theta \in \mathbb{R}$, the right-hand side of the above equation is strictly negative. Step 2 implies then that for $e_{R}$ sufficiently small, no EE exists.

Step 4: Proof of Proposition 3(iii) in the case $w_{R}-w_{L} \rightarrow 0$. Suppose Proposition 3(iii) is false in the case $w_{R}-w_{L} \rightarrow 0$. Then from Step 2, there exists $\left(w^{k}\right)_{k \in \mathbb{N}}$ such that $w_{R}^{k}-w_{L}^{k} \rightarrow 0$ and for all $k \in \mathbb{N}$, for all $w^{*, k} \in W^{*}\left(w^{k}\right), e_{R}^{*}\left(w^{*, k}, e_{L}^{*}\left(w^{*, k}, e_{L}\right), e_{R}\right)<0$. Taking differences across players in (14), we obtain $w_{R}^{*, k}-w_{L}^{*, k}=\frac{w_{R}^{k}-w_{L}^{k}}{1-\delta\left(F\left(w_{R}^{*, k}\right)-F\left(w_{L}^{*, k}\right)\right)} \leq$ $\frac{w_{R}^{k}-w_{L}^{k}}{1-\delta}$, so $w_{R}^{*, k}>w_{L}^{*, k}$ and $w_{R}^{*, k}-w_{L}^{*, k} \rightarrow_{k \rightarrow \infty} 0$. Since $w_{R}^{*, k}>w_{L}^{*, k}$, (15) for $i=R$ implies $e_{R}^{*}\left(w^{*, k}, \underline{e_{L}^{*}}\left(w^{*, k}, e_{L}\right), e_{R}\right) \geq e_{R}+\delta \int_{w_{L}^{*, k}}^{w_{*}^{*, k}} b_{R}(\theta, q)\left(\theta-w_{R}^{*, k}\right) d F(\theta)$. Since $w_{R}^{*, k}-$ $w_{L}^{*, k} \rightarrow_{k \rightarrow \infty} 0$, continuity of $F$ implies that the right-hand side of the above inequality tends to $e_{R}>0$ as $k \rightarrow \infty$, which contradicts that $e_{R}^{*}\left(w^{*, k}, e_{L}^{*}\left(w^{*, k}, e_{L}\right), e_{R}\right)<0$.

The next lemma will be used to prove Proposition 3(iii).

Lemma 4. If $\sigma$ is an equilibrium, then

$$
\begin{aligned}
& e_{i}^{\sigma}=e_{i}+\delta \int_{\Upsilon^{\sigma}(q, q)} e_{i}^{\sigma} d \mu(v)+\int_{\Upsilon^{\sigma}(p, p) \cap \Upsilon^{\sigma}(q, n)}\left(\theta(v)-w_{i}^{\sigma}\right) d \mu(v) \\
& w_{i}^{\sigma}=w_{i}+\delta \int_{\Upsilon^{\sigma}(p, p) \cap \Upsilon^{\sigma}(n, n)}\left(w_{i}^{\sigma}-\theta(v)\right) d \mu(v)-\delta \int_{\Upsilon^{\sigma}(n, q)} e_{i}^{\sigma} d \mu(v) .
\end{aligned}
$$


and for some $D$ such that $(1-\delta)^{2} \leq D \leq 1$,

$$
\begin{aligned}
w_{R}^{\sigma}-w_{L}^{\sigma}= & \frac{\left(1-\delta \mu\left(\Upsilon^{\sigma}(q, q)\right)\right)\left[w_{R}-w_{L}\right]-\delta \mu\left(\Upsilon^{\sigma}(n, q)\right)\left[e_{R}-e_{L}\right]}{D}, \\
e_{R}^{\sigma}-e_{L}^{\sigma}= & \left(1-\delta \mu\left(\Upsilon^{\sigma}(p, p) \cap \Upsilon^{\sigma}(n, n)\right)\right)\left[e_{R}-e_{L}\right] / D \\
& -\delta \mu\left(\Upsilon^{\sigma}(p, p) \cap \Upsilon^{\sigma}(q, n)\right)\left[w_{R}-w_{L}\right] / D, \\
w_{R}^{\sigma}+e_{R}^{\sigma}-w_{L}^{\sigma}-e_{L}^{\sigma}= & \left(1-\delta \mu\left(\Upsilon^{\sigma}(p, p) \cap \Upsilon^{\sigma}(q,\{n, q\})\right)\right)\left[w_{R}-w_{L}\right] / D \\
& +\left(1-\delta \mu\left(\Upsilon^{\sigma}(p, p) \cap\left\{\Upsilon^{\sigma}(n, n) \cup \Upsilon^{\sigma}(n, q)\right\}\right)\right)\left[e_{R}-e_{L}\right] / D .
\end{aligned}
$$

Proof. Step 1: For any equilibrium $\sigma$, $\Upsilon^{\sigma}(q, q) \subset \Upsilon^{\sigma}(p, p), \Upsilon^{\sigma}(n, q) \subset \Upsilon^{\sigma}(p, p)$, and (23), and (22) hold. That $Y^{\sigma}(q, q) \subset \Upsilon^{\sigma}(p, p)$ in EE follows from Lemma 3A. Suppose now that $\sigma$ is an IE. In any $v \in \Upsilon^{\sigma}(q, q)$, some player weakly prefer implementing $q$ to $n$, so from Lemma 2A, $\theta(v) \geq \min _{k \in\{L, R\}}\left\{w_{k}^{\sigma}+e_{k}^{\sigma}\right\}$. Using part (iii) of Proposition 2, the last inequality implies that $\theta(v)>\min _{k \in\{L, R\}} w_{k}^{\sigma}$, so one player must strictly prefer implementing $p$ to $n$ and, therefore, $v \notin \Upsilon^{\sigma}(p, n)$. From part (ii) of Proposition 2, $e_{i}^{\sigma}>0$, so from Lemma 2A, $v \notin \Upsilon^{\sigma}(p, q)$. Since $\left(\Upsilon^{\sigma}(p, x)\right)_{x \in\{n, p, q\}}$ is a partition of $\Upsilon$, this implies $v \in \mathrm{Y}^{\sigma}(p, p)$, as needed.

To show $\Upsilon^{\sigma}(n, q) \subset \Upsilon^{\sigma}(p, p)$, let $v \in \Upsilon^{\sigma}(n, q)$. Since $\sigma$ is an equilibrium, in state $\theta(v)$ both players must weakly prefer implementing $q$ to $n$, so from Lemma 3A, $\theta(v) \geq$ $\max _{i \in\{L, R\}}\left\{w_{i}^{\sigma}+e_{i}^{\sigma}\right\}$. Using Lemma 3A (for an EE) and part (iii) of Proposition 2 (for an IE), we have

$$
\max _{i \in\{L, R\}}\left\{w_{i}^{\sigma}+e_{i}^{\sigma}\right\} \geq \min _{i \in\{L, R\}}\left\{w_{i}^{\sigma}+e_{i}^{\sigma}\right\}>\min _{i \in\{L, R\}}\left\{w_{i}^{\sigma}\right\},
$$

so $\theta(v)>\min _{i \in\{L, R\}}\left\{w_{i}^{\sigma}\right\}$, which means that one player strictly prefers implementing $p$ to $n$ and, therefore, $v \notin \Upsilon^{\sigma}(p, n)$. Since $\Upsilon^{\sigma}(p, q)=\varnothing$, and since $\left(\Upsilon^{\sigma}(p, x)\right)_{x \in\{n, p, q\}}$ is a partition of $\Upsilon$, necessarily, $v \in \Upsilon^{\sigma}(p, p)$, as needed.

Substituting $\Upsilon^{\sigma}(n, q) \subset \Upsilon^{\sigma}(p, p)$ into (7), we obtain (23). Finally, by definition of $\Upsilon^{\sigma}, \Upsilon^{\sigma}(p, n) \cap \Upsilon^{\sigma}(p, p)=\varnothing$, so $\Upsilon^{\sigma}(q, q) \subset \Upsilon^{\sigma}(p, p)$ implies $\Upsilon^{\sigma}(p, n) \cap \Upsilon^{\sigma}(q, q)=\varnothing$. Substituting the latter equality and $\Upsilon^{\sigma}(q, q) \subset \Upsilon^{\sigma}(p, p)$ into (8), we obtain (22).

Step 2: Proof of the first two lines of (24). Subtracting (23) for $i=R$ from (23) for $i=L$, and doing the same for (22), we get

$$
\left\{\begin{array}{l}
w_{R}^{\sigma}-w_{L}^{\sigma}=w_{R}-w_{L}+\delta \mu\left(\Upsilon^{\sigma}(p, p) \cap \Upsilon^{\sigma}(n, n)\right)\left(w_{R}^{\sigma}-w_{L}^{\sigma}\right)-\delta \mu\left(\Upsilon^{\sigma}(n, q)\right)\left(e_{R}^{\sigma}-e_{L}^{\sigma}\right) \\
e_{R}^{\sigma}-e_{L}^{\sigma}=e_{R}-e_{L}+\delta \mu\left(\Upsilon^{\sigma}(q, q)\right)\left(e_{R}^{\sigma}-e_{L}^{\sigma}\right)-\delta \mu\left(\Upsilon^{\sigma}(p, p) \cap \Upsilon^{\sigma}(q, n)\right)\left(w_{R}^{\sigma}-w_{L}^{\sigma}\right) .
\end{array}\right.
$$

The above equations can be viewed as a linear system in $w_{R}^{\sigma}-w_{L}^{\sigma}$ and $e_{L}^{\sigma}-e_{R}^{\sigma}$. Straightforward algebra shows that its solution is given by the first two lines of (24) for

$$
\begin{aligned}
D \equiv & \left(1-\delta \mu\left(\Upsilon^{\sigma}(p, p) \cap \Upsilon^{\sigma}(n, n)\right)\right)\left(1-\delta \mu\left(\Upsilon^{\sigma}(q, q)\right)\right) \\
& -\delta^{2} \mu\left(\Upsilon^{\sigma}(p, p) \cap \Upsilon^{\sigma}(q, n)\right) \mu\left(\Upsilon^{\sigma}(n, q)\right) .
\end{aligned}
$$


Step 3: Proof of the third line of (24). From Step 1, $\Upsilon^{\sigma}(q, q) \subset \Upsilon^{\sigma}(p, p)$ and, by definition of $\Upsilon^{\sigma}, \Upsilon^{\sigma}(q, n)$ and $\Upsilon^{\sigma}(q, q)$ are disjoint, so

$$
\mu\left(\Upsilon^{\sigma}(p, p) \cap \Upsilon^{\sigma}(q, n)\right)+\mu\left(\Upsilon^{\sigma}(q, q)\right)=\mu\left(\Upsilon^{\sigma}(p, p) \cap\left\{\Upsilon^{\sigma}(q, n) \cup \Upsilon^{\sigma}(q, q)\right\}\right) .
$$

From Step $1, \Upsilon^{\sigma}(n, q) \subset \Upsilon^{\sigma}(p, p)$ and, by definition of $\Upsilon^{\sigma}$, $\Upsilon^{\sigma}(n, n)$ and $\Upsilon^{\sigma}(n, q)$ are disjoint, so

$$
\mu\left(\Upsilon^{\sigma}(p, p) \cap \Upsilon^{\sigma}(n, n)\right)+\mu\left(\Upsilon^{\sigma}(n, q)\right)=\mu\left(\Upsilon^{\sigma}(p, p) \cap\left\{\Upsilon^{\sigma}(n, n) \cup \Upsilon^{\sigma}(n, q)\right\}\right) .
$$

Adding up the first two lines of (24) and substituting (25) and (26) into the corresponding expression for $w_{R}^{\sigma}+e_{R}^{\sigma}-w_{L}^{\sigma}-e_{L}^{\sigma}$, we obtain the third line of (24) for the above $D$.

Step 4: $(1-\delta)^{2} \leq D \leq 1$. That $D \leq 1$ is obvious from the definition of $D$. To prove $D \geq(1-\delta)^{2}$, observe that $\Upsilon^{\sigma}(q, q)$ and $\Upsilon^{\sigma}(p, p) \cap \Upsilon^{\sigma}(q, n)$ are disjoint. So $\Upsilon^{\sigma}(q, q)$ is included into the complement of $\Upsilon^{\sigma}(p, p) \cap \Upsilon^{\sigma}(q, n)$. Likewise, $\Upsilon^{\sigma}(p, p) \cap \Upsilon^{\sigma}(n, n)$ and $\Upsilon^{\sigma}(n, q)$ are disjoint, so $\Upsilon^{\sigma}(p, p) \cap \Upsilon^{\sigma}(n, n)$ is included in the complement of $\Upsilon^{\sigma}(n, q)$. Therefore,

$$
\begin{aligned}
D= & \left(1-\delta \mu\left(\Upsilon^{\sigma}(p, p) \cap \Upsilon^{\sigma}(n, n)\right)\right)\left(1-\delta \mu\left(\Upsilon^{\sigma}(q, q)\right)\right) \\
& -\delta^{2} \mu\left(\Upsilon^{\sigma}(p, p) \cap \Upsilon^{\sigma}(q, n)\right) \mu\left(\Upsilon^{\sigma}(n, q)\right) \\
\geq & \left(1-\delta\left(1-\mu\left(\Upsilon^{\sigma}(n, q)\right)\right)\right)\left(1-\delta\left(1-\mu\left(\Upsilon^{\sigma}(p, p) \cap \Upsilon^{\sigma}(q, n)\right)\right)\right) \\
& -\delta^{2} \mu\left(\Upsilon^{\sigma}(p, p) \cap \Upsilon^{\sigma}(q, n)\right) \mu\left(\Upsilon^{\sigma}(n, q)\right) \\
= & (1-\delta)\left(1-\delta+\delta\left(\mu\left(\Upsilon^{\sigma}(n, q)\right)+\mu\left(\Upsilon^{\sigma}(p, p) \cap \Upsilon^{\sigma}(q, n)\right)\right)\right) \geq(1-\delta)^{2} .
\end{aligned}
$$

Proof of Proposition 3 part (iii) in the case $w_{R}-w_{L} \rightarrow+\infty$. Suppose to the contrary that part (iii) is false in the case $w_{R}-w_{L} \rightarrow+\infty$. Then there exists $e \in(0,+\infty)^{2}$, $m \in \mathbb{R}$, and two sequences $\left(w^{k}\right)_{k \in \mathbb{N}}$ and $(\sigma(k))_{k \in \mathbb{N}}$ with the properties $w_{R}^{k}-w_{L}^{k} \rightarrow+\infty$ for all $k \in \mathbb{N}, w_{L}^{k}+w_{R}^{k}=m$, and $\sigma(k)$ is an IE for the flow-payoff parameters $\left(e, w^{k}\right)$. Below, we show that for $k$ sufficiently large, $e_{L}^{\sigma(k)}>0$ and $e_{R}^{\sigma(k)}>0$, which, from Lemma 3, contradicts the assumption that $\sigma(k)$ is an IE.

Step 1: for $k$ sufficiently large, $w_{L}^{\sigma(k)}<w_{L}^{\sigma(k)}+e_{L}^{\sigma(k)}<w_{R}^{\sigma(k)}+e_{R}^{\sigma(k)} \leq w_{R}^{\sigma(k)}$. Since $w_{R}^{k}-w_{L}^{k} \rightarrow+\infty$, (24) and inequality $D \geq(1-\delta)^{2}$ in Lemma 4 imply that, for $k$ large enough,

$$
w_{R}^{\sigma(k)}-w_{L}^{\sigma(k)} \geq(1-\delta)\left(w_{R}^{k}-w_{L}^{k}\right)-\left|e_{R}-e_{L}\right| .
$$

Hence, $w_{R}^{\sigma(k)}-w_{L}^{\sigma(k)} \rightarrow+\infty$, so for $k$ large enough, $w_{R}^{\sigma(k)}>w_{L}^{\sigma(k)}$, and parts (i) and (ii) of Proposition 2 imply that $e_{L}^{\sigma(k)}>0 \geq e_{R}^{\sigma(k)}$. Moreover, (24) and inequality $D \geq(1-\delta)^{2}$ in Lemma 4 imply

$$
w_{R}^{\sigma(k)}+e_{R}^{\sigma(k)}-w_{L}^{\sigma(k)}-e_{L}^{\sigma(k)} \geq(1-\delta)\left(w_{R}^{k}-w_{L}^{k}\right)-\left|e_{R}-e_{L}\right| .
$$

So $w_{R}^{\sigma(k)}+e_{R}^{\sigma(k)}-w_{L}^{\sigma(k)}-e_{L}^{\sigma(k)} \rightarrow+\infty$. Together with $e_{L}^{\sigma(k)}>0 \geq e_{R}^{\sigma(k)}$, this implies Step 1. 
Step 2: For $k$ sufficiently large,

$$
e_{R}^{\sigma(k)} \geq e_{R}+\delta \mu\left(\Upsilon^{\sigma(k)}(q, q)\right) e_{R}^{\sigma(k)}+\delta \int_{w_{L}^{\sigma(k)}}^{w_{L}^{\sigma(k)}+e_{L}^{\sigma(k)}}\left(w_{L}^{\sigma(k)}-w_{R}^{\sigma(k)}\right) d F(\theta) .
$$

Let $v \in \Upsilon^{\sigma(k)}(p, p) \cap \Upsilon^{\sigma(k)}(q, n)$. Since $\sigma(k)$ is an equilibrium, in state $v$, both players must weakly prefer implementing $n$ to $q$ and one player must prefer implementing $p$ to $n$. From Lemma 2A, this means that $\min _{i \in\{L, R\}} w_{i}^{\sigma(k)} \leq \theta(v) \leq \min _{i \in\{L, R\}}\left(w_{i}^{\sigma(k)}+\right.$ $\left.e_{i}^{\sigma(k)}\right)$. Together with Step 1, this implies

$$
\Upsilon^{\sigma(k)}(p, p) \cap \Upsilon^{\sigma(k)}(q, n) \subset\left\{v \in \Upsilon: w_{L}^{\sigma(k)} \leq \theta(v) \leq w_{L}^{\sigma(k)}+e_{L}^{\sigma(k)}\right\} .
$$

From (27), for all $v \in \Upsilon^{\sigma(k)}(p, p) \cap \Upsilon^{\sigma(k)}(q, n), \theta(v)-w_{L}^{\sigma(k)} \leq e_{L}^{\sigma(k)}$. Substituting this inequality into (22), we obtain that

$$
e_{L}^{\sigma(k)} \leq e_{L}+\delta \mu\left(\Upsilon^{\sigma(k)}(q, q)\right) e_{L}^{\sigma(k)}+\delta \mu\left(\Upsilon^{\sigma(k)}(p, p) \cap \Upsilon^{\sigma(k)}(q, n)\right) e_{L}^{\sigma(k)}
$$

and, therefore, that $e_{L}^{\sigma(k)} \leq e_{L} /(1-\delta)$. Since $e_{L}^{\sigma(k)}>0$, the latter inequality implies that $e_{L}^{\sigma(k)}$ is bounded. From Step 1 and (27), for all $v \in \Upsilon^{\sigma(k)}(p, p) \cap \Upsilon^{\sigma(k)}(q, n), 0 \geq \theta(v)-$ $w_{R}^{\sigma(k)} \geq w_{L}^{\sigma(k)}-w_{R}^{\sigma(k)}$. The preceding inequality and (27) imply that

$$
\int_{\Upsilon^{\sigma}(p, p) \cap \Upsilon^{\sigma}(q, n)}\left(\theta(v)-w_{R}^{\sigma}\right) d \mu(v) \geq \int_{w_{L}^{\sigma(k)}}^{w_{L}^{\sigma(k)}+e_{L}^{\sigma(k)}}\left(w_{L}^{\sigma(k)}-w_{R}^{\sigma(k)}\right) d F(\theta) .
$$

Substituting the above inequality into (22), we obtain Step 2.

Step 3: As $k \rightarrow+\infty, w_{L}^{\sigma(k)} \rightarrow-\infty, w_{R}^{\sigma(k)} \rightarrow+\infty$, and $w_{R}^{\sigma(k)} \sim\left|w_{L}^{\sigma(k)}\right|$. As shown in Step 1, $w_{R}^{k}-w_{L}^{k} \rightarrow+\infty$. Therefore, to prove Step 3, it suffices to show that $w_{L}^{\sigma(k)}+w_{R}^{\sigma(k)}$ is bounded as $k \rightarrow \infty$. To do so, note that if we sum (22) and (23) across players and collect the terms in the factor of $w_{L}^{\sigma(k)}+w_{R}^{\sigma(k)}$ and $e_{L}^{\sigma(k)}+e_{R}^{\sigma(k)}$, we obtain

$$
\begin{gathered}
\left(\begin{array}{cc}
1-\delta \mu\left(\Upsilon^{\sigma(k)}(p, p) \cap \Upsilon^{\sigma(k)}(n, n)\right) & \delta \mu\left(\Upsilon^{\sigma(k)}(n, q)\right) \\
\delta \mu\left(\Upsilon^{\sigma(k)}(p, p) \cap \Upsilon^{\sigma(k)}(q, n)\right) & 1-\delta \mu\left(\Upsilon^{\sigma(k)}(q, q)\right)
\end{array}\right)\left(\begin{array}{c}
w_{L}^{\sigma(k)}+w_{R}^{\sigma(k)} \\
e_{L}^{\sigma(k)}+e_{R}^{\sigma(k)}
\end{array}\right) \\
=\left(\begin{array}{cc}
m-\delta \int_{\Upsilon^{\sigma(k)}(p, p) \cap \Upsilon^{\sigma(k)}(n, n)} \theta(v) d \mu(v) \\
e_{L}+e_{R}+\int_{Y^{\sigma(k)}(p, p) \cap Y^{\sigma(k)}(q, n)} \theta(v) d \mu(v)
\end{array}\right) .
\end{gathered}
$$

Using the notations and results in Lemma 4 , the determinant of that system is $D$, which is bounded away from 0 as $k \rightarrow \infty$. Moreover, all the coefficients of the above system are bounded. Therefore, the solution $w_{L}^{\sigma(k)}+w_{R}^{\sigma(k)}$ must be bounded as $k \rightarrow \infty$, as needed.

Step 4: For $k$ sufficiently large, $e_{L}^{\sigma(k)}>0$ and $e_{R}^{\sigma(k)}>0$. Step 1 establishes that for $k$ large enough, $e_{L}^{\sigma(k)}>0$. Let us now prove that $e_{R}^{\sigma(k)}>0$. Step 3 together with the integrability of $F$ implies that $\int_{w_{L}^{\sigma(k)}}^{w_{L}^{\sigma(k)}+e_{L}^{\sigma(k)}} w_{L}^{\sigma(k)} d F(\theta) \rightarrow 0$. Substituting this limit in Step 2, we obtain $e_{R}^{\sigma(k)}>0$, as needed. 
Proof of Proposition 4. The strategy of the proof follows the intuition provided in the main text after Proposition 4. We show that one can pick $[\underline{\delta}, \bar{\delta}]$ and $[\underline{m}, \bar{m}]$ such that if the aforementioned sequence of $\operatorname{EE}(\sigma(k))_{k \in \mathbb{N}}$ exists, then $w_{L}^{\infty}<m<\min \left\{w_{L}^{\infty}+\right.$ $\left.e_{L}^{\infty}, w_{R}^{\infty}\right\}$. The latter inequalities mean that as $k \rightarrow \infty$, with probability arbitrarily close to 1 , the state is such that players agree to repeal $q$ (because $m<\min \left\{w_{L}^{\infty}+e_{L}^{\infty}, w_{R}^{\infty}+e_{R}^{\infty}\right\}$ ) but $R$ prefers to repeal $p$, whereas $L$ prefers to leave it in place (because $w_{L}^{\infty}<m<w_{R}^{\infty}$ ). Given such expectations, for $k$ sufficiently large, $e_{R}^{\sigma(k)}<0$. That is, $R$ prefers to intervene with $q$ than with $p$, a contradiction with the assumption that $\sigma(k)$ is an EE.

Step 1: There exists $\underline{\delta}, \bar{\delta}, \underline{m}, \bar{m}$ such that $0<\underline{\delta}<\bar{\delta}<1$ and

$$
w_{L}<\underline{m}<\bar{m}<w_{L}+\min \left\{(1-\bar{\delta}) e_{L}, w_{R}-w_{L}-\frac{(1-\underline{\delta}) e_{R}}{\underline{\delta} \underline{b}}\right\}<w_{R} .^{25}
$$

Since $w_{R}-w_{L}>0$, for $\underline{\delta}$ sufficiently close to $1, \min \left\{(1-\bar{\delta}) e_{L}, w_{R}-w_{L}-\frac{(1-\underline{\delta}) e_{R}}{\underline{\delta b}}\right\}>0$, so by continuity, we can find $\underline{\delta}<\bar{\delta}<1$ for which the interval $\left(w_{L}, w_{L}+\min \left\{(1-\bar{\delta}) e_{L}, w_{R}-\right.\right.$ $\left.\left.w_{L}-\frac{(1-\delta) e_{R}}{\underline{\delta} \underline{b}}\right\}\right)$ is nonempty and, hence, we can find $\underline{m}$ and $\bar{m}$ such that the first three inequalities in (28) are satisfied. To prove the last inequality, note that $w_{L}+\min \{(1-$ $\left.\bar{\delta}) e_{L}, w_{R}-w_{L}-\frac{(1-\underline{\delta}) e_{R}}{\underline{\delta b}}\right\} \leq w_{R}-\frac{(1-\underline{\delta}) e_{R}}{\underline{\delta b}}<w_{R}$.

$$
\begin{gathered}
\text { Step 2: Let } \pi^{\infty} \equiv \lim _{k \rightarrow \infty} \int_{w_{L}^{\sigma(k)}}^{\min \left\{w_{L}^{\sigma(k)}+e_{L}^{\sigma(k)}, w_{R}^{\sigma(k)}\right\}} b_{R}(\theta, q) d F_{m_{k}, d_{k}}(\theta) \geq 0 . \text { Then } \\
w_{i}^{\infty}=w_{i}+\frac{\delta}{1-\delta}\left(w_{i}-m\right) \\
e_{i}^{\infty}=e_{i}-\frac{\delta \pi^{\infty}}{1-\delta}\left(w_{i}-m\right) .
\end{gathered}
$$

Since we suppose that $\sigma(k)$ are EE for all $k \in \mathbb{N}$, from Lemma $3 \mathrm{~A}$, we have for all $k \in \mathbb{N}$,

$$
w_{i}^{\sigma(k)}=w_{i}+\delta_{k} \int_{w_{L}^{\sigma(k)}}^{w_{R}^{\sigma(k)}}\left(w_{i}^{\sigma(k)}-\theta\right) d F_{m_{k}, d_{k}}(\theta)
$$

and $w_{L}^{\sigma(k)}<w_{L}<w_{R}<w_{R}^{\sigma(k)}$. As $d_{k} \rightarrow 0$, the support of $F_{k}$ becomes increasingly concentrated around $m$ and from Step 1, $w_{L}<\underline{m} \leq m \leq \bar{m}<w_{R}$. So if we let $k \rightarrow \infty$ in (31), we obtain $w_{i}^{\infty}=w_{i}+\delta\left(w_{i}^{\infty}-m\right)$, which implies (29). From Lemma 3B, we can assume without loss of generality (w.l.o.g.) that $\sigma(k)$ satisfies (13), so

$$
e_{i}^{\sigma(k)}=e_{i}+\delta_{k} \int_{w_{L}^{\sigma(k)}}^{\min \left\{w_{L}^{\sigma(k)}+e_{L}^{\sigma(k)}, w_{R}^{\sigma(k)}\right\}} b_{R}(\theta, q)\left[(\theta-m)+\left(m-w_{i}^{\sigma(k)}\right)\right] d F_{m_{k}, d_{k}}(\theta) .
$$

If we let $k \rightarrow \infty$ in (32) and use the definition of $\pi^{\infty}$, we obtain $e_{i}^{\infty}=e_{i}+\delta \pi^{\infty}\left(m-w_{i}^{\infty}\right)$. Substituting (29) into the latter equation, we obtain (30).

Step 3: $e_{R}^{\infty}<0$. Summing (29) and (30) for $i=L$, we obtain

$$
w_{L}^{\infty}+e_{L}^{\infty}=w_{L}+e_{L}+\frac{\delta\left(1-\pi^{\infty}\right)}{1-\delta}\left(w_{L}-m\right)
$$

\footnotetext{
${ }^{25}$ Recall that $\underline{b}$ is the lower bound on the function $b_{i}(\cdot, \cdot)$.
} 
From Step 1, $m \geq \underline{m}>w_{L}$, so the right-hand side of (33) is increasing in $\pi^{\infty}$ and since $\pi^{\infty} \geq 0$, (33) implies

$$
w_{L}^{\infty}+e_{L}^{\infty} \geq \frac{w_{L}+(1-\delta) e_{L}-\delta m}{1-\delta} .
$$

From Step 1, $m<w_{L}+(1-\delta) e_{L}$. Substituting this inequality into the right-hand side of (34), we obtain $w_{L}^{\infty}+e_{L}^{\infty}>m$. Since $w_{L}^{\infty}<m<w_{R}^{\infty}$, this implies that $w_{L}^{\infty}<m<$ $\min \left\{w_{L}^{\infty}+e_{L}^{\infty}, w_{R}^{\infty}\right\}$. Using the latter inequalities in the definition of $\pi^{\infty}$, we obtain $\pi^{\infty} \geq \underline{b}$. From Step $1, m<w_{R}-\frac{(1-\delta) e_{R}}{\delta \underline{b}}$, so $w_{R}-m>\frac{(1-\delta) e_{R}}{\delta \underline{b}}$. Substituting the latter inequality into (30), we obtain $e_{R}^{\infty}<e_{R}\left(1-\frac{\pi^{\infty}}{\underline{b}}\right)<0$, where the latter inequality follows from $\pi^{\infty} \geq \underline{b}$.

Proof of Proposition 5. Parts A and B below prove part (i); parts $\mathrm{C}$ and D prove part (ii); part E proves part (iii).

Part A: If (3) and (4) hold, there exists an equilibrium of $\Gamma(n, p, q)$ and of $\Gamma(n, q)$ that is Pareto superior to any equilibrium of $\Gamma(n, p)$. Assume (3) and (4) hold, and define the strategy profile $\sigma^{*}(n, p, q)$ of $\Gamma(n, p, q)$ as follows: Under status quo $n$ or $q$, in state $\underline{\theta}(\bar{\theta})$, both players propose and accept $n(q)$, veto $q(n)$, and in both states, $R$ vetoes $p$ and $L$ accepts $p$. Under status quo $p, L$ proposes $p$ and accepts only $p$, whereas $R$ proposes $p$ and accepts any proposal. The path of $\sigma^{*}(n, p, q)$ is as follows: Under status quo $n$ or $q$, $n$ is implemented in state $\underline{\theta}$ and $q$ is implemented in state $\bar{\theta}$, and once $p$ is implemented, $p$ stays in place forever.

Below, we characterize the equilibria of $\Gamma(n, p)$ (Step A1), show that both players strictly prefer $\sigma^{*}(n, p, q)$ to any equilibrium of $\Gamma(n, p)$ (Step A2), and show that $\sigma^{*}(n, p, q)$ is an equilibrium of $\Gamma(n, p, q)$ (Steps A3-A6). We then show that there exists an equilibrium $\sigma^{*}(n, q)$ of $\Gamma(n, q)$ that has the same path as $\sigma^{*}(n, p, q)$ and, thus, that also Pareto dominates any equilibrium of $\Gamma(n, p)$ (Step A7).

Step A1: In any equilibrium of $\Gamma(n, p)$, the initial status quo $n$ stays in place forever. Let $\sigma$ be an equilibrium of $\Gamma(n, p)$. From (3), $w_{L}<\underline{\theta}$, so $L$ gets a strictly greater flowpayoff from $p$ than from any other policy in either state. Therefore, on the path of $\sigma$, once implemented, $p$ stays in place forever. Simple algebra shows that (4) means that in state $\bar{\theta}$ (and, thus, in state $\underline{\theta}$ as well), $R$ strictly prefers implementing $n$ forever to implementing $p$ forever. Therefore, in either state, given continuation play $\sigma, R$ strictly prefers implementing $n$ forever to $p$ forever. Therefore, the path of $\sigma$ stays at the initial status quo $n$ forever.

Step A2: Both players strictly prefer $\sigma^{*}(n, p, q)$ to any equilibrium of $\Gamma(n, p)$. The path of $\sigma^{*}(n, p, q)$ implements $n$ and $q$ in states $\underline{\theta}$ and $\bar{\theta}$, respectively. From (3), both players get a strictly greater payoff from $q$ than from $n$ in state $\bar{\theta}$, so they strictly prefer the path of $\sigma^{*}(n, p, q)$ to staying at $n$ forever. Step A2 follows then from Step A1.

Step A3: Given continuation play $\sigma^{*}(n, p, q)$, in either state, $L$ strictly prefers implementing $p$ to either $n$ or $q$. On the path of $\sigma^{*}(n, p, q), p$ stays in place forever once implemented, and from (3), $p$ gives a strictly greater flow-payoff to $L$ than any other policy in any state. 
Step A4: Given continuation play $\sigma^{*}(n, p, q)$, both players prefer implementing $n$ to $q$ ( $q$ to $n$ ) in state $\underline{\theta}(\bar{\theta})$. From (3), both players get a weakly greater flow-payoff from $n$ than from $q$ (from $q$ than from $n$ ) in state $\underline{\theta}(\bar{\theta})$ and the same continuation value as under $\sigma^{*}(n, p, q)$, status quo $n$ or $q$ leads to the same outcome.

Step A5: Given continuation play $\sigma^{*}(n, p, q)$, in any state, $R$ strictly prefers implementing $n$ or $q$ to $p$. From Steps A1 and A2, $R$ prefers implementing $n$ and playing $\sigma^{*}(n, p, q)$ thereafter to implementing $n$ forever. Moreover, (4) means that in either state, $R$ strictly prefers $n$ forever to $p$ forever, which is what happens if $p$ is implemented and $\sigma^{*}(n, p, q)$ is played thereafter. Therefore, given continuation play $\sigma^{*}(n, p, q)$, in either state, $R$ strictly prefers implementing $n$ to implementing $p$. From Step A4, in state $\bar{\theta}$, $R$ strictly prefers implementing $q$ to $n$, so from the preceding sentence, she must strictly prefer implementing $q$ to $p$ in state $\bar{\theta}$. Since the flow-payoff difference between $q$ and $p$ is independent of $\theta, R$ also strictly prefers implementing $q$ to $p$ in state $\underline{\theta}$.

Step A6: $\sigma^{*}(n, p, q)$ is an equilibrium of $\Gamma(n, p, q)$ and it is Pareto superior to any equilibrium of $\Gamma(n, p)$. Given the preferences characterized in Steps A3-A5, the behavior prescribed by $\sigma^{*}(n, p, q)$ is sequentially rational, both players strictly prefer its path to the unique equilibrium path of $\Gamma(n, p)$.

Step A7: There exists an equilibrium of $\Gamma(n, q)$ that is Pareto superior to any equilibrium of $\Gamma(n, p)$. By construction, under status quo $n$ or $q, \sigma^{*}(n, p, q)$ never prescribes a player to make proposal $p$. So $\sigma^{*}(n, p, q)$ defines also an equilibrium of $\Gamma(n, q)$, and A7 follows from A2.

Part B: If there exists an equilibrium of $\Gamma(n, p, q)$ or $\Gamma(n, q)$ that is Pareto superior to any equilibrium $\sigma^{*}(n, p)$ of $\Gamma(n, p)$, then (3) and (4) must hold. Let $\sigma(n, p, q)$ be an equilibrium of $\Gamma(n, p, q)$ that is Pareto superior to any equilibrium of $\Gamma(n, p)$ (the argument for the case of $\Gamma(n, q)$ is analogous and is omitted for brevity).

Step B1: $w_{L}<\underline{\theta}$. Suppose first that $w_{L} \geq \underline{\theta}$ and $w_{R} \geq \bar{\theta}$. In that case, player $R$ gets a weakly greater flow-payoff from $n$ than from $p$ in either state, so there exists an equilibrium of $\Gamma(n, p)$ in which $R$ unilaterally decides to stay forever at the initial status quo $n$. This equilibrium implements the most preferred policy path of $R$ and, thus, cannot be dominated by any equilibrium of $\Gamma(n, p, q)$, a contradiction. Suppose now that $w_{L} \geq \underline{\theta}$ and $w_{R}<\bar{\theta}$. In that case, both players get a greater flow-payoff from $p(n)$ than from $n$ $(p)$ in state $\bar{\theta}(\underline{\theta})$, so there exists an equilibrium of $\Gamma(n, p)$ in which players agree to implement $n$ in state $\underline{\theta}$ and $p$ in state $\bar{\theta}$. This equilibrium path is optimal for both players and can, thus, not be dominated by any equilibrium of $\Gamma(n, p, q)$, a contradiction.

Step B2: $w_{R}>\underline{\theta}$. Suppose $w_{R} \leq \underline{\theta}$. Then both players get a weakly greater flowpayoff from $p$ than from $n$ in either state of nature. So there exists an equilibrium of $\Gamma(n, p)$ in which both players' most preferred alternative $p$ is implemented forever, so this equilibrium cannot be dominated by any equilibrium of $\Gamma(n, p, q)$, a contradiction.

Step B3: Given continuation play $\sigma(n, p, q), L$ strictly prefers implementing $p$ to $n$ and $p$ to $q$, so under status quo $p$, $p$ stays in place forever. Step B3 follows from the observation that since $w_{L}<\underline{\theta}, L$ gets a strictly greater flow-payoff from $p$ than from any other policy in either state of nature.

Step B4: Given continuation play $\sigma(n, p, q), R$ weakly prefers implementing $q$ to $p$ in either state, and $R$ strictly prefers implementing $n$ to $q$ and $n$ to $p$ in state $\underline{\theta}$. Observe first 
that $q$ must be implemented on the path of $\sigma(n, p, q)$; otherwise $\Gamma(n, p)$ would admit an equilibrium with the same path as $\sigma(n, p, q)$, so those equilibria would be payoff equivalent. From Step B3, $L$ must veto $q$ under status quo $p$, so $q$ must be implemented with positive probability in some state $\theta^{*} \in\{\underline{\theta}, \bar{\theta}\}$ under status quo $n$. Since from Step B3, $L$ strictly prefers implementing $p$ to $q, R$ must weakly prefer implementing $q$ to $p$ in state $\theta^{*}$. Since the flow-payoff difference between $p$ and $q$ and the distribution of future states is independent of the current state, this must be the case in either state, which proves the first claim of Step B4. Since $q$ gives a strictly lower flow-payoff than $p$ to $R$, the first claim of Step B4 implies that $R$ strictly prefers status quo $q$ to status quo $p$. So it must be that in some state $\theta^{\prime}$, with positive probability, status quo $q$ leads to some outcome $x$ that $R$ strictly prefers to $p$ and $q$. So $x$ must be $n$. From the first claim of Step B4, this implies that $R$ strictly prefers implementing $n$ to $p$ in state $\theta^{\prime}$. Since the flow-payoff gain from $n$ relative to $p$ or $q$ is decreasing in $\theta$, this is true for $\theta^{\prime}=\underline{\theta}$.

Step B5: Given continuation play $\sigma(n, p, q), R$ strictly prefers implementing $n$ to $p$ in any state, so under status quo $n$, $p$ is never implemented. Suppose Step B5 is false. Since from Step B4, $R$ strictly prefers $n$ to $p$ in $\underline{\theta}$, it must be that $R$ weakly prefers implementing $p$ to $n$ in state $\bar{\theta}$. Using Step B3, this means that in state $\bar{\theta}, R$ weakly prefers implementing $p$ forever to $n$ forever, since $R$ can unilaterally impose the latter policy path under status quo $n$. Since $R$ gets a greater flow-payoff from $n$ than from $p$ in state $\underline{\theta}$, and since $L$ gets a strictly greater flow-payoff from $p$ than from $n$ in either state, this implies that there exists an equilibrium of $\Gamma(n, p)$ in which the initial status quo $n$ stays in place as long as the state stays at $\underline{\theta}$, and $p$ is implemented at the first occurrence of $\bar{\theta}$ and stays in place forever after. This equilibrium implements $L$ 's most preferred policy path from the first occurrence of state $\bar{\theta}$ onward. By assumption, $L$ is strictly better off under $\sigma(n, p, q)$. For that to be the case, it must be that at the initial status quo $n$ and in state $\underline{\theta}, \sigma(n, p, q)$ implements $q$ or $p$ with positive probability, a contradiction to Step B4.

Step B6: Under status quo $n$ or $q, p$ is never implemented on the path of $\sigma(n, p, q)$. Suppose Step B6 is false. From Step B5, this implies that $p$ is implemented with positive probability under status quo $q$. From Step B4, this means that given continuation play $\sigma(n, p, q), R$ is indifferent between implementing $q$ or $p$ in any state. So from Step B5, $R$ strictly prefers implementing $n$ to $p$ and to $q$ in any state, so $R$ must weakly prefer staying at $n$ forever to playing $\sigma(n, p, q)$. Since she can unilaterally impose $n$ forever in $\Gamma(n, p)$, she must weakly prefer any equilibrium of $\Gamma(n, p)$ to $\sigma(n, p, q)$, a contradiction.

Step B7: Given continuation play $\sigma(n, p, q)$, both players strictly prefer implementing $q$ to $n$ in state $\bar{\theta}$. Suppose Step B7 is false. Given the flow-payoff specification, this means that some player weakly prefers implementing $n$ to $q$ in either state. From Step B6, this implies that she must weakly prefer staying at $n$ forever to playing $\sigma(n, p, q)$. Since she can unilaterally impose $n$ forever in $\Gamma(n, p)$, she must weakly prefer any equilibrium of $\Gamma(n, p)$ to $\sigma(n, p, q)$, a contradiction.

Step B8: With probability 1 , status quo $n$ stays in place in $\underline{\theta}$ and is replaced by $q$ in $\bar{\theta}$. That $n$ stays in place in state $\underline{\theta}$ follows immediately from Step B4. That it is replaced by $q$ with probability 1 follows from Steps B5 and B7.

Step B9: Status quo q stays in place with probability 1 in state $\bar{\theta}$; in state $\underline{\theta}$, it is replaced with positive probability by $n$ and stays in place with the remaining probability. That $q$ 
stays in place with probability 1 in state $\bar{\theta}$ follows from Steps B5 and B7. Suppose now that status quo $q$ is never replaced by $n$ in state $\underline{\theta}$. From Step B6, this implies that $q$ must stay in place with probability 1 . But then both players strictly prefer implementing $p$ forever, so $\sigma(n, p, q)$ is not an equilibrium, a contradiction.

Step B10: Condition (3) must be satisfied. The first inequality of (3) comes from Step B1. For the path of play described by Steps B8 and B9 to be an equilibrium, both players must get a weakly greater (smaller) flow-payoff from $n$ than from $q$ in state $\theta(\bar{\theta})$. This means that the last three inequalities in (3) must hold weakly. Suppose to the contrary that the last inequality holds with equality. Then one player must be indifferent between staying at $n$ forever and the equilibrium path. Since she can unilaterally impose $n$ forever in the game $\Gamma(n, p)$, she must weakly prefer any equilibrium of $\Gamma(n, p)$ to $\sigma(n, p, q)$, a contradiction.

Step B11: Condition (4) must be satisfied. Consider the strategy profile $\sigma(n, p)$ of $\Gamma(n, p)$ defined as follows: $L$ always proposes $p$ and accepts only $p$, and $R$ proposes $n$ $(p)$ and accepts only $n(p)$ in state $\underline{\theta}(\bar{\theta})$. The path of play of $\sigma(n, p)$ is such that $n$ stays in place until the first occurrence of state $\bar{\theta}$, at which time $n$ is replaced by $p$ and $p$ stays in place forever. Since the path of play of $\sigma(n, p)$ implements $n$ until the first occurrence of state $\bar{\theta}$ and never implements $p, L$ must strictly prefers $\sigma(n, p)$ to $\sigma(n, p, q)$, so by assumption, $\sigma(n, p)$ cannot be an equilibrium of $\Gamma(n, p)$. From Step B1, $R$ gets a strictly greater flow-payoff from $p$ than from $n$ in either state, so the actions prescribed by $\sigma(n, p)$ to $L$ are subgame perfect given continuation play $\sigma(n, p)$. The actions prescribed by $\sigma(n, p)$ to $R$ under status quo $p$ are also subgame perfect since $R$ is not pivotal under that status quo. From Step B2, $R$ gets a greater flow-payoff from $n$ than from $p$ in state $\underline{\theta}$, so in that state, it is subgame perfect for $R$ not to propose $p$ and to veto $p$ under status quo $n$. The only remaining case is when the status quo is $n$ and the state is $\bar{\theta}$. Since $\sigma(n, p)$ is not an equilibrium, it must be that $R$ 's actions in that case are not subgame perfect. This means that in state $\bar{\theta}, R$ must strictly prefer staying at $n$ forever than switching to $p$ forever. Simple algebra shows that this condition is equivalent to (4).

Part C: If all equilibria of $\Gamma(n, p, q)$ are Pareto superior to any equilibrium of $\Gamma(n, p)$, then (3), (4), and (5) must hold. Suppose all equilibria of $\Gamma(n, p, q)$ are Pareto superior to any equilibrium of $\Gamma(n, p)$. We already know from part B that (3) and (4) must be satisfied. So to prove part C, it suffices to prove (5). To do so, consider the following strategy profile $\sigma^{\prime}(n, p, q)$. Under status quo $p$, both players propose $p, L$ accepts only $p$, and $R$ vetoes only $q$. Under status quo $n$, both players propose $n, R$ accepts only $n$, and $L$ vetoes only $q$. Under status quo $q$, both players proposes $p$, and $L$ accepts only $p$, whereas $R$ accepts any policy. By construction, the path of play of $\sigma^{\prime}(n, p, q)$ implements $n$ forever. Since either player can unilaterally impose that outcome in the game $\Gamma(n, p), \sigma^{\prime}(n, p, q)$ is not Pareto superior to any equilibrium of $\Gamma(n, p)$, so by assumption, it cannot be an equilibrium. In what follows, we assume that (5) is violated, we characterize players' incentives given continuation play $\sigma^{\prime}(n, p, q)$ (Step C1), and we show that $\sigma^{\prime}(n, p, q)$ is an equilibrium (Step C2), a contradiction.

Step C1: If condition (5) is violated, then given continuation play $\sigma^{\prime}(n, p, q)$, in either state, $R$ prefers implementing $n$ to $p$ to $q$, whereas $L$ prefers implementing $p$ to $q$ to $n$. Note first that since status quo $q$ and $p$ both lead to policy $p$, and since $p$ gives 
both players a greater flow-payoff than $q$, both players prefer implementing $p$ to $q$ in any state. Moreover, (4) implies that in either state, $R$ prefers implementing $n$ forever to $p$ forever, which is what happens if $n$ and $p$ are implemented, respectively, given continuation play $\sigma^{\prime}(n, p, q)$. Therefore, $R$ prefers implementing $n$ to $p$ to $q$. To complete the proof, it remains to show that $L$ prefers implementing $q$ to $n$ in either state, given continuation play $\sigma^{\prime}(n, p, q)$ or, equivalently, that $L$ prefers implementing $q$ today and $p$ forever to implementing $n$ forever. Simple algebra shows that this is equivalent to (5) being violated.

Step C2: If condition (5) is violated, $\sigma^{\prime}(n, p, q)$ is an equilibrium. It is straightforward to check that given the preferences characterized in Step C1, $\sigma^{\prime}(n, p, q)$ prescribes either veto players to accept (reject) a proposal whenever it gives her a greater (lesser) continuation payoff, and it prescribe either proposer to propose the policy that gives her the greatest continuation payoff among those accepted by the veto player, as needed.

Part D: If (3), (4), and (5) hold, any equilibrium of $\Gamma(n, p, q)$ or $\Gamma(n, q)$ is strictly Pareto superior to any equilibrium of $\Gamma(n, p)$. Assume (3), (4), and (5). The strategy profile $\sigma^{*}(n, p, q)$ defined in part A is an equilibrium that is strictly Pareto better than any equilibrium of $\Gamma(n, p)$. So it suffices to show that any equilibrium of $\Gamma(n, p, q)$ and $\Gamma(n, q)$ must have the same path as $\sigma^{*}(n, p, q)$. In Steps D1-D6 below, $\sigma$ is an arbitrary equilibrium of $\Gamma(n, p, q)$.

Step D1: Given continuation play $\sigma$, in either state, status quo p always stays in place and $L$ strictly prefers implementing $p$ to $n$ or $q$. From (3), $p$ gives a strictly greater flowpayoff to $L$ than any other policy in any state. So $R$ strictly prefers staying forever at $p$ to any other policy path, and she can unilaterally impose that continuation path once $p$ is the status quo.

Step D2: Given continuation play $\sigma$, in either state, $R$ strictly prefers implementing $n$ to $p$. Once $n$ is the status quo, $R$ can unilaterally impose to stay at $n$ forever. Since $\sigma$ is an equilibrium, this implies that $R$ must weakly prefer implementing $n$ today and playing $\sigma$ thereafter to implementing $n$ forever. Moreover, from (4), in any state, $R$ strictly prefers implementing $n$ forever to $p$ forever, which, from Step D1, is what happens if $p$ is implemented and $\sigma$ is played thereafter. Step D2 follows then by transitivity.

Step D3: $p$ is never implemented on the path of $\sigma$. From Step D1, $p$ is never implemented under status quo $n$. So if Step D3 is false, it must be that (i) in some state $\theta^{\prime}, p$ is implemented with positive probability under status quo $q$ and that (ii) in some state $\theta^{\prime \prime}, q$ is implemented with positive probability under the initial status quo $n$. Note that (i) implies that given continuation play $\sigma$, in state $\theta^{\prime}, R$ weakly prefers implementing $p$ to implementing $q$. Since the flow-payoff of $q$ relative to $p$ is independent of $\theta$, this must also be true in state $\theta^{\prime \prime}$. Likewise, (ii) implies that $R$ weakly prefers implementing $q$ to $n$ in state $\theta^{\prime \prime}$. By transitivity, $R$ weakly prefers implementing $p$ to $n$ in state $\theta^{\prime \prime}$, a contradiction with Step D2.

Step D4: Given continuation play $\sigma$, in state $\underline{\theta}$, both players strictly prefer implementing $n$ to $q$, so in state $\underline{\theta}$, status quo $n$ or $q$ leads to $n$. Suppose to the contrary that given continuation play $\sigma$, some player $i$ prefers implementing $q$ to $n$ in state $\underline{\theta}$. Since the flowpayoff of $n$ relative to $q$ is decreasing in $\theta, i$ prefers implementing $q$ to $n$ in both states, so from Step D3, $i$ weakly prefers implementing $q$ forever to implementing $n$ forever. But 
(4) means that in any state, $R$ strictly prefers implementing $n$ forever to $p$ forever and, thus, to $q$ forever. So $i$ must be $L$. Simple algebra shows that (5) implies that in state $\underline{\theta}, L$ prefers implementing $n$ forever to implementing $q$ today and $p$ forever after, so $L$ must prefer $n$ forever to $q$ forever, a contradiction. The second claim in Step D4 follows from the first claim and Step D3.

Step D5: Given continuation play $\sigma$, in state $\bar{\theta}$, both players strictly prefer implementing $q$ to $n$, so in state $\bar{\theta}$, status quo n or q leads to policy $q$. From Step D4, in state $\underline{\theta}$, status quos $n$ and $q$ lead to the same outcome. Moreover, in state $\bar{\theta}$, from (3), both players get a strictly greater flow-payoff from $q$ than from $n$. Therefore, given continuation play $\sigma$, both players strictly prefer implementing $q$ to $n$. The second claim in Step D5 follows from the first claim and Step D3.

Step D6: $\sigma$ has the same path as $\sigma^{*}(n, p, q)$. This step follows directly from Steps D3, D4, and D5.

Step D7: Any equilibrium of $\Gamma(n, q)$ has the same path as $\sigma^{*}(n, p, q)$. This step follows directly from the proof of Steps D4 and D5.

Part E: For any $\left(w_{L}, w_{R}, e_{L}, e_{R}\right)$, there exists $(\delta, \underline{\theta}, \bar{\theta}, \pi)$ such that (3), (4), and (5) hold. Pick $\underline{\theta}$ and $\bar{\theta}$ so that (3) holds. Simple algebra shows that (4) and (5) can be rewritten as $\pi<\frac{\bar{w}_{R}-(1-\delta) \bar{\theta}-\delta \underline{\theta}}{\delta(\bar{\theta}-\underline{\theta})}$ and $\pi<\frac{(1-\delta) e_{L}+w_{L}-\underline{\theta}}{\delta(\bar{\theta}-\underline{\theta})}$. The latter two inequalities are satisfied for some $\pi \in(0,1)$ whenever their right-hand sides are strictly positive, which is the case if and only if

$$
\frac{\bar{\theta}-w_{R}}{\bar{\theta}-\underline{\theta}}<\delta<\frac{e_{L}+w_{L}-\underline{\theta}}{e_{L}} .
$$

Note that $\frac{\bar{\theta}-w_{R}}{\bar{\theta}-w_{L}}<1$ and is independent of $\underline{\theta}$, and the right-hand side of (35) is 1 for $\underline{\theta}=$ $w_{L}$. So for any $\bar{\theta}$ satisfying (3), by continuity one can pick $\underline{\theta}$ satisfying (3) and (35).

\section{A.3 Proofs for the model of Section 5}

Lemma 5. Consider the model of Section 5. For every strategy profile $\sigma$, let $V_{i}^{\sigma}$ denote the continuation payoff for player $i \in\{L, R\}$ of implementing policy $x \in\{n, p, q\}$ in some period $t$ with state $(\theta(t), v(t))=(\theta, v) \in \mathbb{R} \times[0,1]$ until the next period in which the state is redrawn, given continuation play $\sigma$. There exist $\left(w_{L}, w_{R}, e_{L}, e_{R}\right) \in \mathbb{R}^{4}$ such that, for any equilibrium strategy profile $\sigma$ and for all $i \in\{L, R\}, \theta \in \mathbb{R}$, and $v \in[0,1]$,

$$
\begin{aligned}
& (1-\delta(1-v))\left[V_{i}^{\sigma}(\theta, v, p)-V_{i}^{\sigma}(\theta, v, n)\right]=\theta-(1-v) w_{i}-v w_{i}^{\sigma} \\
& (1-\delta(1-v))\left[V_{i}^{\sigma}(\theta, v, p)-V_{i}^{\sigma}(\theta, v, q)\right]=(1-v) e_{i}+v e_{i}^{\sigma} .
\end{aligned}
$$

Proof. We use Notation 1 with the exception that a state of the world $v \in \mathcal{Y}$ now also includes the realization of the volatility $v$. For any strategy profile $\sigma$, all $v \in \mathcal{Y}$, and $s \in$ $\{n, p, q\}$, let $X^{\sigma}(v, s)$ denote the policy outcome in a period in which the state of the world is $v$, the status quo is $s$, and players play $\sigma$. By definition of $V_{i}^{\sigma}$,

$$
\begin{aligned}
& V_{i}^{\sigma}(\theta, v, p)-V_{i}^{\sigma}(\theta, v, n) \\
& \quad=\theta-w_{i}+\delta\left\{(1-v)\left[V^{\sigma}(\theta, v, p)-V^{\sigma}(\theta, v, n)\right]\right.
\end{aligned}
$$




$$
\begin{aligned}
& \left.+v \int_{v \in \Upsilon}\left[V^{\sigma}\left(\theta, v, X^{\sigma}(v, p)\right)-V^{\sigma}\left(\theta, v, X^{\sigma}(v, n)\right)\right] d \mu(v)\right\} \\
= & \frac{\theta-(1-v) w_{i}-v\left\{w_{i}-\delta \int_{v \in Y}\left[V^{\sigma}\left(\theta, v, X^{\sigma}(v, p)\right)-V^{\sigma}\left(\theta, v, X^{\sigma}(v, n)\right)\right] d \mu(v)\right\}}{1-\delta(1-v)} .
\end{aligned}
$$

If we set $w_{i}^{\sigma}$ equal to the term inside the curly brackets in the numerator of the above fraction, then $w_{i}^{\sigma}$ depends on neither $\theta$ nor $v$, which proves the first line of (36). The proof for the second line of (36) follows an analogous argument and is omitted for brevity.

Proof of Proposition 6. Let $\sigma$ be an equilibrium. For $q$ to be implemented on some equilibrium path, some player $i$ must weakly prefer implementing $q$ to $p$ in some state $(v, \theta)$ given continuation play $\sigma$. From Lemma 5, we have $V_{i}^{\sigma}(\theta, v, q) \geq V_{i}^{\sigma}(\theta, v, p)$ in some state $(v, \theta) \in[0,1) \times \mathbb{R}$ if and only if $e_{i}^{\sigma}<0$ and $v>\frac{e_{i}}{e_{i}-e_{i}^{\sigma}}$. So if $e_{L}^{\sigma} \geq 0$ and $e_{R}^{\sigma} \geq 0$, for all $(v, \theta) \in[0,1) \times \mathbb{R}, V_{i}^{\sigma}(\theta, v, p)>V_{i}^{\sigma}(\theta, v, q)$, and the first part of Proposition 6 holds trivially for $\bar{v}=1$. Suppose now that $e_{i}^{\sigma}<0$ for some $i$ and let $\bar{v} \equiv \frac{e_{i}}{e_{i}-e_{i}^{\sigma}}<1$. Then from Lemma 5, for all $v<\bar{v}$ and all $\theta \in \mathbb{R}$, both players weakly prefer implementing $p$ to $q$ in state $(v, \theta)$, so $q$ is not implemented on path in such states. When $v>\bar{v}$ and when $\theta$ is sufficiently large, $i$ 's most preferred policy given continuation play $\sigma$ is $q$, and $j$ prefers to accept $q$ under status quo $n$, so $q$ is implemented, as needed.

Consider a sequence of c.d.f.s $\left(H_{k}\right)_{k \in \mathbb{N}}$ such that for all $k \in \mathbb{N}, H_{k}(v, \theta)=G_{k}(v) F(\theta)$, where $G_{k}$ has full support and tends to the degenerate distribution that puts probability 1 on $v=1$ as $k \rightarrow \infty$. Suppose the second claim of Proposition 6 is false, that is, $q$ is never implemented in some equilibrium for any $H_{k}$. This means that for all $k \in \mathbb{N}$, there exists an equilibrium $\sigma(k)$ such that $e_{L}^{\sigma(k)} \geq 0$ and $e_{R}^{\sigma(k)} \geq 0$. Together with (36), the latter inequalities imply that for all $\theta \in \mathbb{R}$ and $k \in \mathbb{N}$, both players strictly (weakly) prefer implementing $p$ to $q$ given continuation play $\sigma(k)$ when $v \in[0,1)$ (when $v=1$ ). So $\sigma(k)$ never prescribes players to propose $q$ when $v \in[0,1)$, and we can assume that this is true as well when $v=1$. Since $G_{k}$ puts probability 0 on $v=1$, this deviation from $\sigma(k)$ does not affect the continuation payoff parameters $w^{\sigma(k)}$ and $e^{\sigma(k)}$, and the modified $\sigma(k)$ is still an equilibrium. Without loss of generality, we can restrict attention to a subsequence of $(\sigma(k))_{k \in \mathbb{N}}$ such that $w^{\sigma(k)}$ and $e^{\sigma(k)}$ converge to some $w^{\infty}$ and $e^{\infty}$.

Let us now show that the corresponding subsequence of $(\sigma(k))_{k \in \mathbb{N}}$ converges. For almost all $(\theta, v), \theta-(1-v) w_{i}-v w_{i}^{\infty} \neq 0$. Together with (36), this implies that for any such $(\theta, v), i$ strictly prefers to implement $p$ to $n$ for all $k$ sufficiently large or $i$ strictly prefers to implement $n$ to $p$ for all $k$ sufficiently large. An analogous statement holds between $n$ and $q$, and between $p$ and $q$. So for almost all $(\theta, v)$, the action prescribed by $\sigma(k)$ to veto player $i$ and, therefore, to proposer $i$, must be pure and constant in $k$ for $k$ sufficiently large.

Note finally that by continuity, $\lim _{k \rightarrow \infty}(\sigma(k))$ must be an equilibrium of $\Gamma$ for the limit c.d.f. The limit of $\left(H_{k}(v, \theta)\right)_{k \in \mathbb{N}}$ as $k \rightarrow \infty$ puts probability 1 on $v=1$, so the limit game is equivalent to the game considered in Proposition 4, and by construction, $q$ is never implemented on path, so it is an EE. Since $\delta$ can be chosen arbitrarily close to 1 and since $F$ can be chosen arbitrarily, we obtain a contradiction with Proposition 4 . 


\section{A.4 Example for statements made in Section 4.1}

Suppose $w_{L} \leq w_{R}$ and $e_{L}<e_{R}$. Below, we build a simple environment with three states $\theta_{1}<\theta_{2}<\theta_{3}$ in which an IE $\sigma$ exists such that $w_{L}^{\sigma}>w_{R}^{\sigma}$ and $e_{L}^{\sigma}<0<e_{R}^{\sigma}$. This equilibrium demonstrates that when $e_{L}<e_{R}$, the players $i$ and $j$ in Proposition 2 can be $R$ and $L$, respectively (see claims at the end of Section 4.1 and in Footnote 16). In the case $w_{L}=w_{R}$, this example also demonstrates that when $e_{L} \neq e_{R}$, for some distribution of the shock, there exists an IE (see the claim in Footnote 17). In these cases, there also obviously exists an EE in which players never disagree between $n$ and $p$ (i.e., $w_{L}^{\sigma}=w_{R}^{\sigma}=w_{L}=w_{R}$ ), so IE and EE can coexist (see the claim in Footnote 18), and in this case the EE is clearly Pareto better than the IE.

The putative equilibrium path of the IE $\sigma$ is as follows. In state $\theta_{1}$, policy makers agree to implement $n$ irrespective of the status quo. A sufficient condition for this is that $\theta_{1}<\min _{i}\left(w_{i}^{\sigma}\right)$. In state $\theta_{2}, n$ stays in place, $q$ is replaced by $n$, and $p$ stays in place. A sufficient condition for this is that $w_{R}^{\sigma}<\theta_{2}<w_{L}^{\sigma}, \theta_{2}<\min _{i}\left(w_{i}^{\sigma}+e_{i}^{\sigma}\right), e_{L}^{\sigma}<0$, and $e_{R}^{\sigma}>0$. In state $\theta_{3}, p$ and $q$ stay in place and $n$ is replaced by $q(p)$ when $L(R)$ is the proposer. A sufficient condition for this is that $w_{R}^{\sigma}<w_{R}^{\sigma}+e_{R}^{\sigma}<\theta_{3}$ and $w_{L}^{\sigma}+e_{L}^{\sigma}<w_{L}^{\sigma}<\theta_{3}$. The aforementioned equilibrium conditions can be summarized as

$$
\left\{\begin{array}{l}
\theta_{1}<w_{R}^{\sigma}<\theta_{2}<w_{R}^{\sigma}+e_{R}^{\sigma}<\theta_{3} \\
\theta_{1}<\theta_{2}<w_{L}^{\sigma}+e_{L}^{\sigma}<w_{L}^{\sigma}<\theta_{3} .
\end{array}\right.
$$

To make sure such an equilibrium exists, we must compute the continuation payoff parameters $\left(w_{i}^{\sigma}, e_{i}^{\sigma}\right)$ using the aforementioned equilibrium path, and check that they satisfy (37). To do so, observe that according to the putative equilibrium path, status quo $p$ $(q)(n)$ leads to policy $n$ being implemented in state $\theta_{1}$, to policy $p(n)(n)$ being implemented in state $\theta_{2}$, and to policy $p(q)$ ( $q$ w.p. $b_{L}$ and $p$ w.p. $b_{L}$ ) being implemented in state $\theta_{3}$. Therefore,

$$
\left\{\begin{array}{l}
e_{i}^{\sigma}=e_{i}+\delta \pi_{2}\left(\theta_{2}-w_{i}^{\sigma}\right)+\delta \pi_{3} e_{i}^{\sigma} \\
\theta-w_{i}^{\sigma}=\theta-w_{i}+\delta \pi_{2}\left(\theta_{2}-w_{i}^{\sigma}\right)+\delta \pi_{3} b_{L} e_{i}^{\sigma} .
\end{array}\right.
$$

Solving these equations for $\left(e_{i}^{\sigma}, w_{i}^{\sigma}\right)$, we obtain

$$
\begin{aligned}
e_{i}^{\sigma} & =\frac{\left(1-\delta \pi_{2}\right) e_{i}+\delta \pi_{2} \theta_{2}-\delta \pi_{2} w_{i}}{1-\delta \pi_{2}-\delta \pi_{3}+\delta^{2} \pi_{2} \pi_{3}\left(1-b_{L}\right)} \\
w_{i}^{\sigma} & =\frac{\left(1-\delta \pi_{3}\right) w_{i}-\delta \pi_{3} e_{i} b_{L}-\delta \theta_{2} \pi_{2}+\delta^{2} \theta_{2} \pi_{2} \pi_{3}\left(1-b_{L}\right)}{1-\delta \pi_{2}-\delta \pi_{3}+\delta^{2} \pi_{2} \pi_{3}\left(1-b_{L}\right)} .
\end{aligned}
$$

For simplicity, fix $\delta=1$ and $b_{L}=1$ in the above expression (if we get the desired strict inequality for $\delta=1$ and $b_{L}=1$, by continuity, we have them for $\delta$ and $b_{L}$ close enough 
to 1$)$. Then we get

$$
\left\{\begin{array}{l}
w_{i}^{\sigma}=w_{i}+\frac{\pi_{2}}{\pi_{1}}\left(w_{i}-\theta_{2}\right)-\frac{\pi_{3}}{\pi_{1}} e_{i}, \\
e_{i}^{\sigma}=\frac{\left(1-\pi_{2}\right) e_{i}+\pi_{2}\left(\theta_{2}-w_{i}\right)}{\pi_{1}}=e_{i}+\frac{\pi_{3}}{\pi_{1}} e_{i}-\frac{\pi_{2}}{\pi_{1}}\left(w_{i}-\theta_{2}\right), \\
w_{i}^{\sigma}+e_{i}^{\sigma}=w_{i}+e_{i} .
\end{array}\right.
$$

The equilibrium conditions in (37) become

$$
\left\{\begin{array}{l}
\theta_{1}<w_{R}+\frac{\pi_{2}}{\pi_{1}}\left(w_{R}-\theta_{2}\right)-\frac{\pi_{3}}{\pi_{1}} e_{R}<\theta_{2}<w_{R}+e_{R}<\theta_{3} \\
\theta_{1}<\theta_{2}<w_{L}+e_{L}<w_{L}+\frac{\pi_{2}}{\pi_{1}}\left(w_{L}-\theta_{2}\right)-\frac{\pi_{3}}{\pi_{1}} e_{L}<\theta_{3} .
\end{array}\right.
$$

We can ignore the outer inequalities, as fixing the remaining parameters, one can always pick $\theta_{1}$ small enough and $\theta_{3}$ large enough such that the remaining inequalities in (38) are also satisfied. The inner inequalities can be rewritten as

$$
\begin{aligned}
& w_{R}-\frac{\pi_{3}}{1-\pi_{3}} e_{R}<\theta_{2}<w_{R}+e_{R} \\
& \theta_{2}<\min \left\{w_{L}+e_{L}, w_{L}-\frac{1-\pi_{2}}{\pi_{2}} e_{L}\right\}=w_{L}-\frac{1-\pi_{2}}{\pi_{2}} e_{L} .
\end{aligned}
$$

Clearly, one can find $\left(w_{R}, w_{L}, e_{R}, e_{L}, \pi_{2}, \pi_{3}, \theta_{2}\right)$ such that these inequalities are satisfied. In particular, set $w_{L}=w_{R}=0$. Then these inequalities require $-\frac{\pi_{3}}{1-\pi_{3}} e_{R}<\theta_{2}<-\frac{1-\pi_{2}}{\pi_{2}} e_{L}$, which can be satisfied as long as $\frac{\pi_{3}}{1-\pi_{3}} e_{R}<\frac{1-\pi_{2}}{\pi_{2}} e_{L}$. The latter inequality is satisfied for some $\pi_{2}$ and $\pi_{3}$ such that $\pi_{2}+\pi_{3}<1$ as long as $e_{R}<e_{L}$, as needed.

\section{REFERENCES}

Acemoglu, Daron and James A. Robinson (2000), "Why did the West extend the franchise? Democracy, inequality, and growth in historical perspective." Quarterly Journal of Economics, 115, 1167-1199. [1489]

Acemoglu, Daron and James A. Robinson (2001), "A theory of political transitions." American Economic Review, 91, 938-963. [1489]

Aidt, Toke S. (2003), “Economic analysis of corruption: A survey." Economic Journal, 113, F632-F652. [1489]

Alesina, Alberto and Silvia Ardagna (2013), “The design of fiscal adjustments.” Tax Policy and the Economy, 27, 19-68. [1487]

Alesina, Alberto and Allan Drazen (1991), "Why are stabilizations delayed?" American Economic Review, 81, 1170-1188. [1488]

Alesina, Alberto, Carlo Favero, Francesco Giavazzi, Omar Barbiero, and Matteo Paradisi (2017), "The effects of fiscal consolidations: Theory and evidence.” Working paper, NBER Working Paper No. 23385. [1487] 
Alesina, Alberto and Francesco Passarelli (2014), "Regulation vs taxation." Journal of Public Economics, 110, 147-156. [1489]

Alesina, Alberto, Roberto Perotti, and J. Tavares (1998), "The political economy of fiscal adjustments.” Brookings Papers on Economic Activity, Spring, 197-266. [1487]

Ali, Nageeb, Maximilian Mihm, and Lucas Siga (2017), "The perverse politics of polarization." Unpublished paper, SSRN 2907489. [1488]

Anesi, Vincent and John Duggan (2018), "Existence and indeterminacy of Markovian equilibria in dynamic bargaining games.” Theoretical Economics, 13, 505-525. [1488]

Anesi, Vincent and Daniel J. Seidmann (2015), "Bargaining in standing committees with an endogenous default.” Review of Economic Studies, 82, 825-867. [1488]

Acharya, Avidit and Juan Ortner (2013): "Delays and partial agreements in multi-issue bargaining." Journal of Economic Theory, 148, 2150-2163. [1488]

Austen-Smith, David, Wioletta Dziuda, Bård Harstad, and Antoine Loeper (2019), "Gridlock and inefficient policy instruments." Unpublished paper, SSRN 2847014. [1501]

Austin, D. Andrew and Mindy Levit (2010), "Mandatory spending since 1962." Congressional Research Service. [1494]

Bagwell, Kyle and Robert Staiger (1990), “A theory of managed trade.” American Economic Review, 80, 779-795. [1486]

Baldursson, Fridrik and Nils-Henrik von der Fehr (2007), "A whiter shade of pale: On the political economy of regulatory instruments." Journal of the European Economic Association, 5, 37-65. [1488]

Bardhan, Pranab (1971), "On the optimum subsidy to a learning industry: An aspect of the theory of infant-industry protection." International Economic Review, 12, 54-70. [1485]

Baron, David P. (1996), “A dynamic theory of collective goods programs.” American Political Science Review, 90, 316-330. [1488]

Becker, Gary and Casey Mulligan (2003), "Deadweight costs and the size of government." Journal of Law and Economics, 46, 293-340. [1489]

Bernheim, B. Douglas, Antonio Rangel, and Luis Rayo (2006), "The power of the last word in legislative policy making." Econometrica, 74, 1161-1190. [1488]

Bowen, Renee, Ying Chen, Hülya Eraslan, and Jan Zápal (2017), "Efficiency of flexible budgetary institutions.” Journal of Economic Theory, 167, 148-176. [1488, 1493]

Bowen, T. Renee, Ying Chen, and Hülya Eraslan (2014), "Mandatory versus discretionary spending: The status quo effect." American Economic Review, 104, 2941-2974. [1488]

Brady, David W. and Craig Volden (2006), Revolving Gridlock: Politics and Policy From Jimmy Carter to George W. Bush, second edition. Routledge, New York. [1488] 
Buisseret, Peter and Dan Bernhardt (2017), "Dynamics of policymaking: Stepping back to leap forward, stepping forward to keep back?” American Journal of Political Science, 61, 820-835. [1488]

Buisseret, Peter and Dan Bernhardt (2018), "Reelection and renegotiation: International agreements in the shadow of the polls?" American Political Science Review, 112, 10161035. [1489]

Canes-Wrone, Brandice, Michael Herron, and Kenneth Shotts (2001), "Leadership and pandering: A theory of executive leadership." American Journal of Political Science, 45, 532-550. [1489]

Coate, Stephen and Stephen Morris (1995), "On the form of transfers to special interests.” Journal of Political Economy, 103, 1210-1235. [1489]

Cox, Gary and Mathew McCubbins (2000), "Political structure and economic policy: The institutional determinants of policy outcomes.” In Presidents, Parliaments and Policy (Stephan Haggard and Mathew McCubbins, eds.). Cambridge University Press, New York, New York. [1488]

Diermeier, Daniel and Pohan Fong (2011), "Legislative bargaining with reconsideration." Quarterly Journal of Economics, 126, 947-985. [1488]

Drazen, Allan and Nuno Limao (2008), "A bargaining theory of inefficient redistribution politics." International Economic Review, 49, 621-657. [1489]

Duggan, John and Tasos Kalandrakis (2012), “Dynamic legislative policy making.” Journal of Economic Theory, 147, 1653-1688. [1488, 1495]

Dziuda, Wioletta and Antoine Loeper (2016), "Dynamic collective choice with endogenous status quo." Journal of Political Economy, 124, 1148-1186. [1484, 1488, 1495]

Dziuda, Wioletta and Antoine Loeper (2018), "Dynamic pivotal politics.” American Political Science Review, 112, 580-601. [1488, 1500]

Fearon, James D. (1998), "Commitment problems and the spread of ethnic conflict." In The International Spread of Ethnic Conflict (David A. Lake and Donald Rothchild, eds.). Princeton University Press, Princeton, New Jersey. Chapter 5. [1489]

Fernandez, Raquel and Dani Rodrik (1991), "Resistance to reform: Status quo bias in the presence of individual-specific uncertainty." American Economic Review, 81, 1146-1155. [1488]

Gale, William and Peter Orszag (2003), "Sunsets in the tax code.” Tax Notes, 99, 15531561. [1494]

Glaeser, Edward L. and Giacomo Ponzetto (2014), "Shrouded costs of government: The political economy of state and local public pensions." Journal of Public Economics, 116, 89-105. [1489]

Grossman, Gene and Elhanan Helpman (1994), "Protection for sale.” American Economic Review, 84, 833-850. [1489] 
Grossman, Gene and Elhanan Helpman (2001), Special Interest Politics. Cambridge, Massachusetts, MIT Press. [1489]

Hassler, John, José Rodríguez Mora, Kjetil Storesletten, and Fabrizio Zillibotti (2003), "The survival of the welfare state." American Economic Review, 93, 87-112. [1488]

Jorgenson, Dale, Richard Goettle, Mun Ho, and Peter Wilcoxen (2013), Double Dividend: Environmental Taxes and Fiscal Reform in the United States. Cambridge, Massachusetts, MIT Press. [1486]

Kalandrakis, Anastassios (2004), "A three-player dynamic majoritarian bargaining game." Journal of Economic Theory, 116, 294-322. [1488]

Krehbiel, Keith (1998), Pivotal Politics: A Theory of US Lawmaking. University of Chicago Press, Chicago. [1488, 1500]

Masciandaro, Donato and Francesco Passarelli (2013), "Financial systemic risk: Taxation or regulation?” Journal of Banking and Finance, 37, 587-596. [1489]

Melitz, Marc (2005), "When and how should infant industries be protected?" Journal of International Economics, 66, 177-196. [1486]

Mooney, Chris (2004), “A short history of sunsets.” Legal Affairs. [1494]

Ortner, Juan (2017), “A theory of political gridlock.” Theoretical Economics, 12, 555-586. [1488]

Posner, Eric and Adrian Vermeule (2002), "Legislative entrenchment: A reappraisal." Yale Law Journal, 111, 1665-1705. [1494]

Powell, Robert (2004), "The inefficient use of power: Costly conflict with complete information.” American Political Science Review, 98, 231-241. [1489]

Riboni, Alessandro and Francisco Ruge-Murcia (2008), "The dynamic (in)efficiency of monetary policy by committee." Journal of Money, Credit, and Banking, 40, 1001-1032. [1488]

Spolaore, Enrico (2004), "Adjustments in different government systems." Economics and Politics, 16, 117-146. [1488]

Strulovici, Bruno (2010), "Learning while voting: Determinants of collective experimentation." Econometrica, 78, 933-971. [1488]

Tullock, Gordon (1967), Towards a Mathematics of Politics. University of Michigan Press, Ann Arbor. [1486]

Tullock, Gordon (1993), Rent Seeking. Edward Elgar Publishing, London. [1489]

Villas-Boas, Miguel (1997), “Comparative statics of fixed points.” Journal of Economic Theory, 73, 183-198. [1516]

Weingast, Barry, Kenneth Shepsle, and Christopher Johnson (1981), "The political economy of benefits and costs: A neoclassical approach to distributive politics." Journal of Political Economy, 89, 642-664. [1488] 
Wright, John R. (1996), Interest Groups and Congress: Lobbying, Contributions, and Influence. Allyn \& Bacon, Boston. Re-issued as Longman Classics in Political Science (Longman, 2003). [1489]

Zapál, Jan (2011), "Explicit and implicit status-quo determination in dynamic bargaining: Theory and application to FOMC directive." Working Paper, CERGE-EI, Prague. [1488]

Co-editor Simon Board handled this manuscript.

Manuscript received 8 June, 2018; final version accepted 10 April, 2019; available online 6 May, 2019. 